\title{
An Ontology for Assembly Representation
}

\author{
Xenia Fiorentini \\ Iacopo Gambino \\ Vei-Chung Liang \\ Sudarsan Rachuri \\ Mahesh Mani \\ Conrad Bock
}


NISTIR 7436

\title{
An Ontology for Assembly Representation
}

\author{
Xenia Fiorentini \\ Iacopo Gambino \\ Vei-Chung Liang \\ Sudarsan Rachuri \\ Mahesh Mani \\ Conrad Bock \\ Manufacturing Systems Integration Division \\ Manufacturing Engineering Laboratory
}

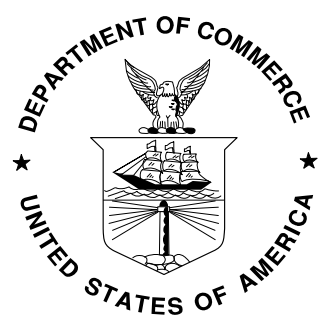

U.S. Department of Commerce Carlos M. Gutierrez, Secretary

National Institute of Standards and Technology James M. Turner, Acting Director 


\section{Abstract}

Mechanical assemblies are systems composed of modules that are either subassemblies or parts. Traditionally an assembly information model contains information regarding parts, their relationships, and its form. But it is important that the model also represent the function and behavior. This report describes the development of an Ontological Assembly Model in the broader context of Product Lifecycle Management (PLM).

The ontological assembly model can help in achieving various levels of interoperability as required to enable the full potential of PLM. In this report we first present an Ontology Web Language (OWL) version of the Core Product Model (CPM) and subsequently an Open Assembly Model (OAM) based on their previous Unified Modeling Language (UML) versions developed at the National Institute of Standards and Technology (NIST) [7] [8] [9]. Besides developing a semantic assembly information model, we further extend this model to incorporate reasoning capabilities. We explore and discuss various tools and methodologies for ontological assembly modeling with reasoning capabilities. The ontological assembly model can be considered an extension to the NIST OAM with semantic interoperability. This extended Assembly Ontology in OWL could serve to test the advantages of a semantic approach to represent a product structure evolution i.e., from the design phases and throughout the life of the product. An example case study is additionally discussed to explain the Ontological Assembly Model including rules and reasoning capabilities. 


\section{Table of Contents}

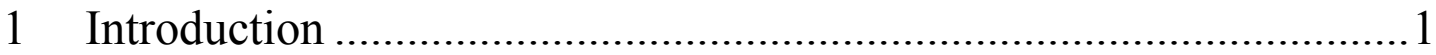

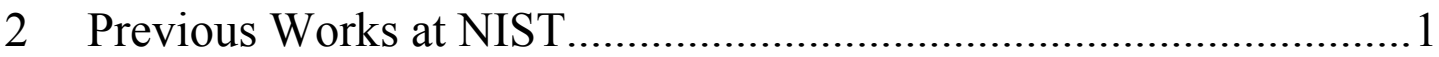

2.1 Core Product Model 1

2.2 Open Assembly Model 6

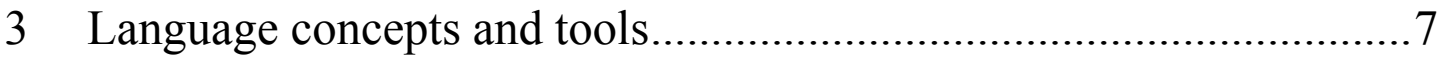

3.1 Patterns for Translation 8

3.1.1 Property Translating Pattern.................................................................. 8

3.1.2 Association Pattern.............................................................................. 10

$\begin{array}{lll}3.2 & \text { Modeling and Reasoning Tools } & 14\end{array}$

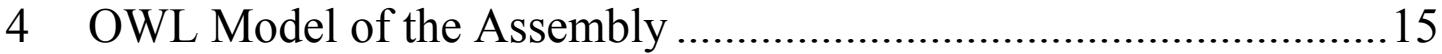

4.1 Translation of the CPM to OWL 15

4.2 Translation of OAM to OWL 17

4.2.1 Relationships between artifacts ............................................................. 17

4.2.2 Relationships between features............................................................. 21

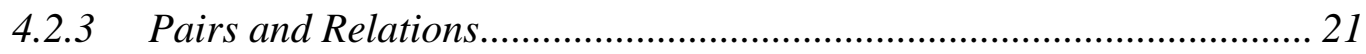

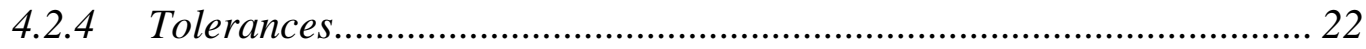

4.2.5 Usage Patterns.............................................................................. 23

5 Reasoning with OWL Assembly Model .......................................26

5.1 Description Logic Reasoning 26

5.2 Rule-based Reasoning 26

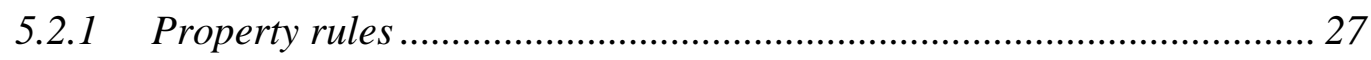

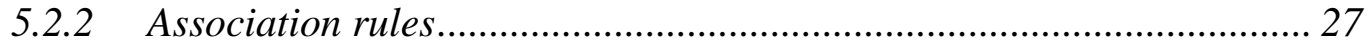

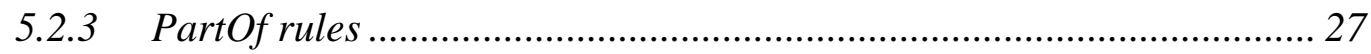

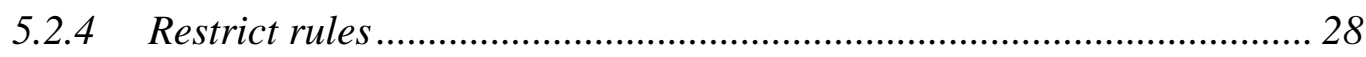

5.3 Rule Analysis and Discussion 29

6 Case study: Planetary Gear System.................................................34

6.1 Use Case Description 34 


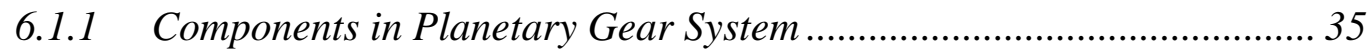

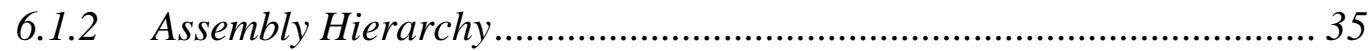

6.2 Use Case Implementation 41

6.2.1 Asserted Instances and Properties.......................................................... 42

6.2.2 Inferred Instances and Properties ....................................................... 51

6.2.3 Kinematic Information Representation................................................. 57

6.2.4 Tolerance Representation in the Planetary Gear System........................ 58

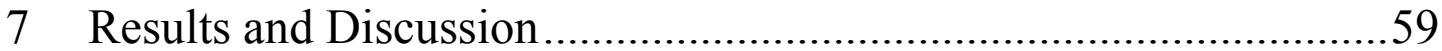

$\begin{array}{lll}7.1 & \text { Model Advantages } & 60\end{array}$

$\begin{array}{lll}7.2 & \text { Limitations and future research directions } & 61\end{array}$

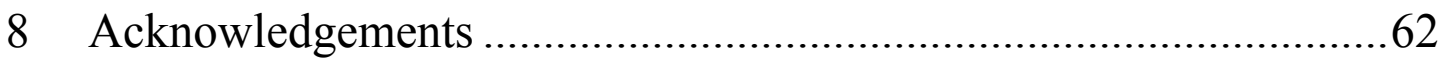

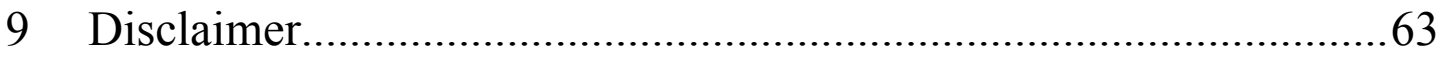

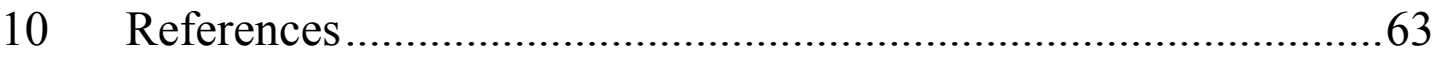

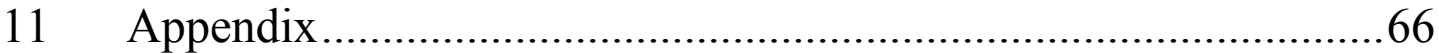




\section{List of Figures}

Figure 1 Class diagram of the Core Product Model ................................................... 3

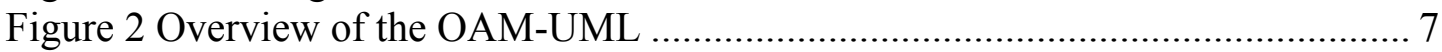

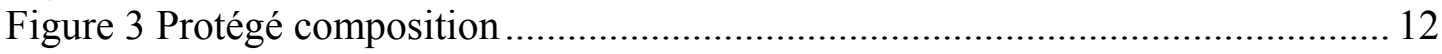

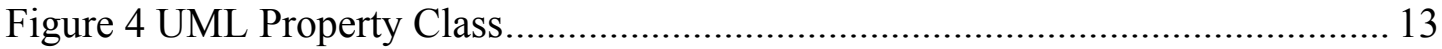

Figure 5 A Property Class connecting two different Object Classes........................... 13

Figure 6 Property pattern for two Object Classes........................................................ 13

Figure 7 First part of the translation of Property Classes ............................................ 14

Figure 8 Second part of the translation of Property Classes........................................ 14

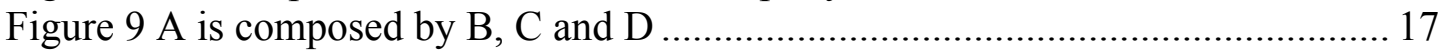

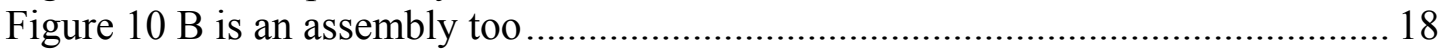

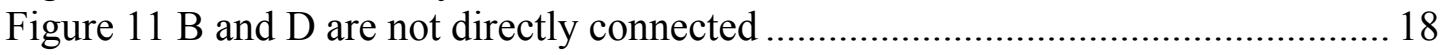

Figure 12 Example of an assembly composition .................................................. 19

Figure 13 The assembly representation in OWL ................................................. 20

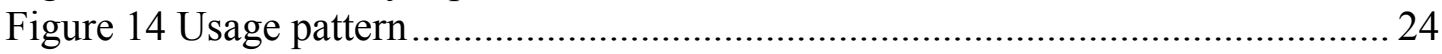

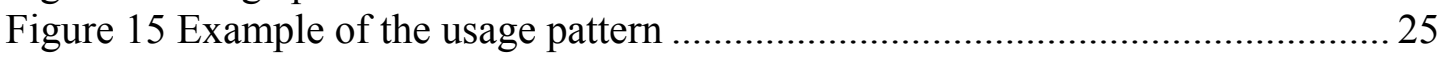

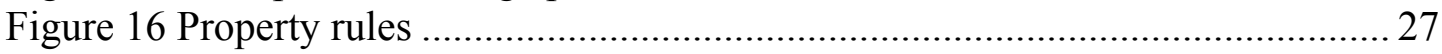

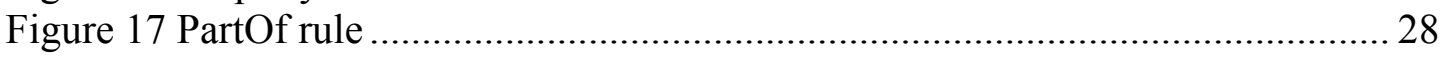

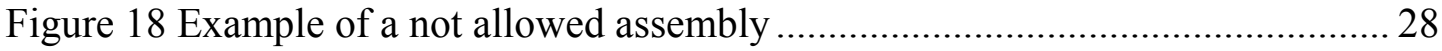

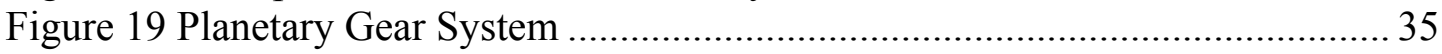

Figure 20 Exploded view of the Planetary Gear System............................................ 35

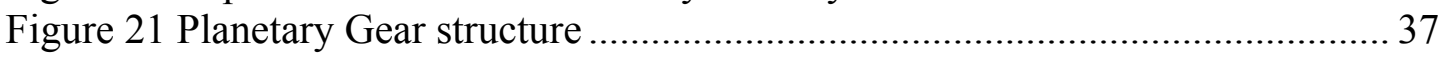

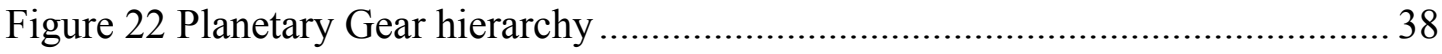

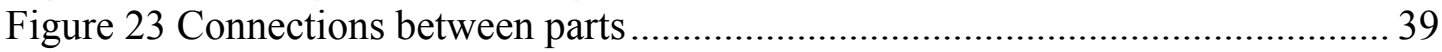

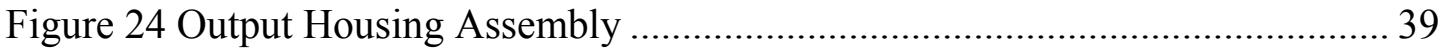

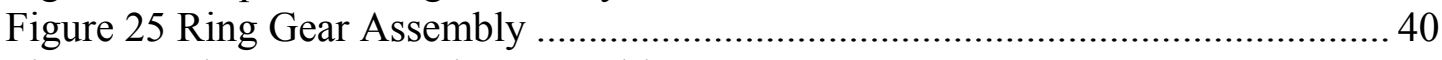

Figure 26 Planet Gear-carrier Assembly .....................................................................4 40

Figure 27 Planet Carrier Assembly and Sungear...................................................... 41

Figure 28 Output Housing Assembly .................................................................... 41

Figure 29 Kinematic Diagram of Planetary Gear System ......................................... 57

Figure 30 Sungear tolerances................................................................................... 59

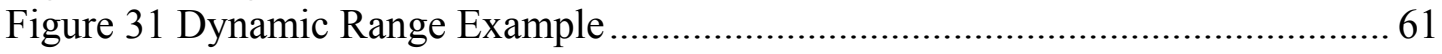

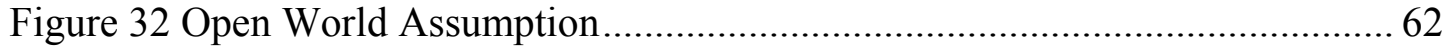




\section{List of Tables}

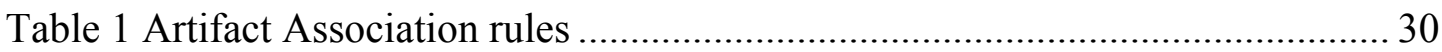

Table 2 Assembly Feature Association rules.............................................................. 31

Table 3 Assembly Feature Association Representation rules..................................... 32

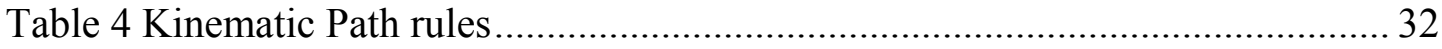

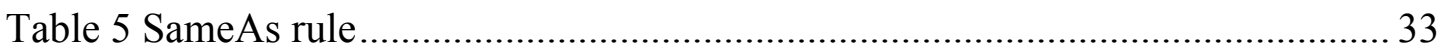

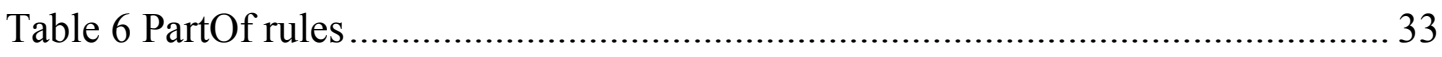

Table 7 Rules for not Allowed Artifacts..................................................................... 34

Table 8 Rule for not allowed Pair Frame.................................................................... 34

Table 9 Components of the Planetary Gear System .................................................... 36

Table 10 Artifact: asserted instances and properties ..................................................... 43

Table 11 Part: asserted instances and properties ………………………………..... 45

Table 12 OAM Features: asserted instances................................................................ 47

Table 13 ArtifactAssociation: asserted instances and properties................................. 48

Table 14 AssemblyFeatureAssociation: asserted instances and properties .................50

Table 15 AssemblyFeatureAssociationRepresentation: asserted instances and

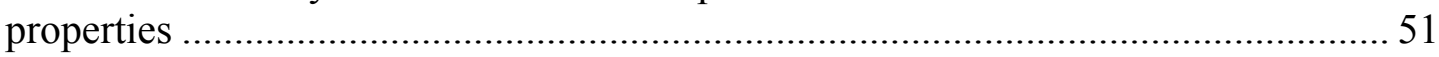

Table 16 Assembly inferred properties...................................................................5 53

Table 17 Meaning_Less_Artifact inferred instances ..................................................53

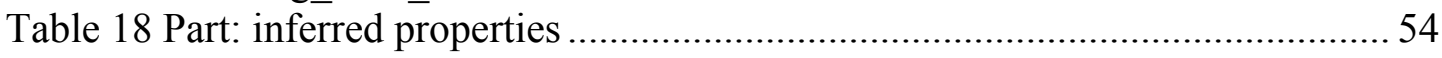

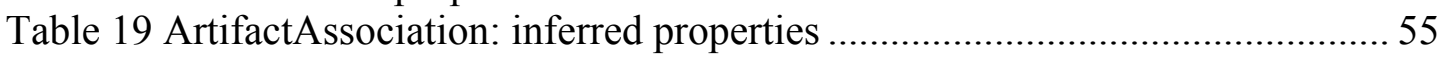

Table 20 AssemblyFeatureAssociation: inferred property ........................................56

Table 21 AssemblyFeatureAssociationRepresentation: inferred properties................5 57

Table 22 Kinematic Pairs and Associated Parts of Planet Gear System ..................... 58 


\section{List of Acronyms}

NIST- National Institute of Standards and Technology

PLM - Product Lifecycle Management

CAD - Computer Aided Design

UML - Unified Modeling Language

OWL - Ontology Web Language

ERP - Enterprise Resource Planning

DSS - Decision Support System

SWRL -Semantic Web Rule Language

CAE- Computer Aided Engineering

URI- Universal Resource Identifier

OAM- Open Assembly Model

CPM- Core Product Model

MOKA- Methodology and tools Oriented to Knowledge-Based Engineering Applications

OWL-DL Ontology Web Language Description Logic

RDF- Resource Description Framework

AFA- Assembly Feature Association

AFAR- Assembly Feature Association Representation 


\section{Introduction}

The development of an ontological assembly representation was initiated from several considerations concerning assembly representation for PLM. The complete distribution and control of information between different stake holders is the underlying goal for the PLM approach. To achieve an interoperability level that could enable efficient implementation of PLM, it is necessary to identify a common data structure to allow data exchange between different stake holder's platforms [1] [2]. A first step towards achieving this goal is to develop information models with standard data structures to support interoperability. An ontology based approach with additional reasoning capabilities could create a new perspective for PLM [3] [4] [5].

In an industrial scenario, many products are assemblies composed of either individual parts or subassemblies produced from different suppliers. An important reason to model assemblies using an ontology is to test the advantages of a semantic approach where the meaning of the modeled concepts is formally defined. The semantic model is especially useful to capture the evolution of the assembly from the design phases and throughout the life of the product. An assembly model is required to represent relationships between artifacts (for example parts, assemblies. We will formally define artifacts in the following sections) that characterize an assembly representation of a product model.

The ontological assembly model can help in achieving various levels of interoperability as required to enable the full potential of PLM. Besides developing a semantic assembly information model, we further extend this model to incorporate reasoning capabilities. This report is organized as follows: Section 2 presents the previous and related work at NIST, here we briefly discuss the UML [6] versions of NIST's CPM [7] [8] and OAM models [9]. Section 3 presents useful methodologies and relevant tools used for the creation of the ontology. Section 4 presents the OWL [10] model of the assembly representation. Section 5 presents the reasoning capabilities of the ontology representation. Section 6 presents an implemented case study to explain the Ontological Assembly Model including the applied rules and reasoning capabilities. Finally Section 7 summarizes the report with results and discussion.

\section{Previous Works at NIST}

NIST's CPM and OAM [9] are a starting point towards developing an ontological assembly model. CPM is a product representation model while OAM is the CPM extension for an assembly representation. Both CPM and OAM were originally UML models [11] [12]. In this section a brief overview of the UML versions of CPM and OAM are presented for better understanding.

\subsection{Core Product Model}

The CPM [7] [8] was intended to form a base for future systems that could respond to the demands of the next generation CAD systems besides providing improved 
interoperability among future software. CPM is an abstract model with generic or meaningful semantics about a particular domain to be embedded within an implementation model and the policy of use of that model. The key concept that makes CPM a candidate for supporting the full range of PLM activities is that a product is described by a triad:

- Function: what the artifact is supposed to do; the term function is often used synonymously with the term intended behavior.

- Form: the proposed design solution for the design problem specified by the function; in CPM, the artifact's physical characteristics are modeled in terms of its geometry (the "traditional" domain of CAD models) and material properties.

- Behavior: how the artifact implements its function in terms of the engineering principles incorporated into a behavioral or causal model; application of the behavioral model to the artifact describes or simulates the artifact's observed behavior based on its form [13].

Figure 1 shows a UML diagram [14] [6] of the CPM composed of four categories of classes: classes that provide supporting information for the objects (abstract classes), physical or conceptual objects classes, classes that describe associations (relationships) among the objects and classes that are commonly used by other classes. For more information please refer to Fenves et al, 2001.

In the rest of this report, the following naming conventions are used: names of CPM classes are written in boldface and capitalized (e.g., CoreProductModel, EntityAssociation, Artifact). Names of attributes are in boldface and lower case (e.g., information) while names of instances are in italics (e.g., cylindricalForm).

The common information is stored in five supporting classes: exploring the model starting from the highest level of generalization, the first class CoreProductModel represents the highest level of generalization. For this class we define the common attributes type, name and information and they are inherited by all the classes of the model.

CommonCoreObject is the base class for all the object classes. CommonCoreRelationship and its specializations may be applied to instances of classes derived from this class.

CommonCoreRelationship is the base class from which all association classes are specialized. 


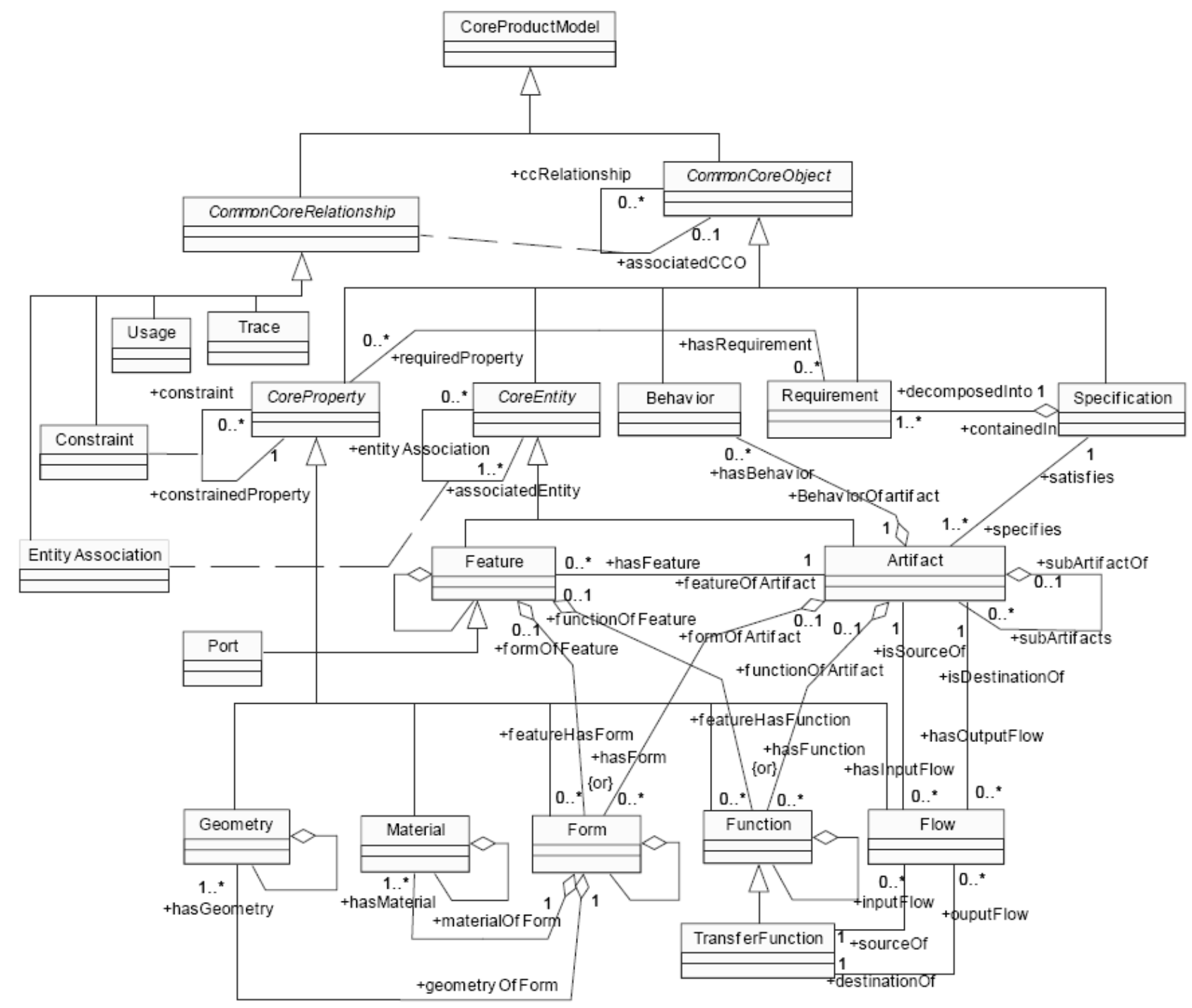

Figure 1 Class diagram of the Core Product Model

CoreEntity is the base class from which the classes Artifact and Feature are specialized. EntityAssociation relationships may be applied to entities in this class.

CoreProperty is an abstract class from which the classes Function, Flow, Form, Geometry and Material are specialized. Constraint relationships may be applied to instances of this class.

The following constitute the object classes:

Artifact is the key object class in the model. It represents a distinct entity in a product, whether a component, part, subassembly or assembly. All the latter entities can be represented and interrelated through the subArtifacts/subArtifactof containment hierarchy. 
Feature is a portion of the artifact's form that has some specific function assigned to it. Thus, an artifact may have design features, analysis features, manufacturing features, etc. Feature has its own containment hierarchy, so that compound features can be created out of other features. A Feature has attributes of Function and Form, but does not have a different Behavior since its Behavior is defined in the Artifact containing the Feature.

Port is a specific kind of feature, sometimes referred to an interface feature.

Specification is the collection of information relevant to an Artifact deriving from customer needs and/or engineering requirements; it is a container for the specific Requirements that the artifact must satisfy.

Requirement is a specific element of the Specification of an artifact that governs some aspect of its function, form, geometry or material. Requirements cannot be applied to Behavior, which is strictly determined by a behavioral model. This is because requirements represent what the artifact is supposed to do while Behavior is the observed performance of the Artifact.

Function represents what the artifact or feature is supposed to do. The artifact satisfies customer needs and/or engineering requirements largely through its function.

TransferFunction is a specialized form of Function involving the transfer of an input flow into an output flow.

Flow is the medium that serves as the output of one or more transfer function(s) and the input of one or more other transfer function(s).

Behavior describes how the artifact implements its function; it is governed by physical, chemical or other engineering principles that are incorporated into a behavioral or causal model.

Form of the artifact or feature is the design solution for the problem specified by the function. In the CPM, the artifact's or feature's physical characteristics are represented by two distinct classes, namely:

Geometry is the spatial description of an artifact or feature.

Material is the material composition of an artifact or feature.

The following constitutes the association (relationship) classes derived from the CommonCoreRelationship class:

Constraint is a specific shared property of a set of entities that must hold in all cases. At the level of the CPM, only the entity instances that constitute the constrained set are identified.

EntityAssociation is a simple set membership relationship among artifacts and features. 
Usage is a mapping from CommonCoreObject to CommonCoreObject, useful when constraints apply to multiple "target" entities but not to the generic "source" entity.

Trace is structurally identical to Usage, useful when the "target" entity in the current product description depends on a "source" entity in another product description.

The following three utility classes (not shown on Figure 1) provide additional detail: Information, an attribute of CoreProductModel and all its specializations, is a container consisting of three attributes: a textual description, a textual documentation and properties that represent a set of attribute-value pairs representing all domain or object-specific attributes. process_information, an attribute of Artifact, contains product development process parameters that may be used in a PLM environment. Rationale, an attribute of CoreProperty, documents decision in the product development process.

The classes described above are linked by three kinds of associations.

First, all object classes have their own separate, independent decomposition hierarchies by attributes such as subArtifacts/subArtifactof for the Artifact class.

Second, there are associations between:

- a Specification and the Artifact that results from it

- a Flow and its source, destination Artifacts and its input/ output Functions

- a Artifact and its Features

Third, and most importantly, four aggregations are fundamental to the CPM:

- Function, Form and Behavior aggregate into Artifact

- Function and Form aggregate into Feature

- Geometry and Material aggregate into Form

- Requirement aggregates into Specification.

The conceptual model of CPM may be used in actual applications. Specific instances of entities must be located by means of their type and their attributes stored in and retrieved from the properties slot of the associated Information instance. These same two constructs, type and properties, may be used by a model compiler to create subclasses of Artifact from the specifications in the type slot, and define attributes on the subclasses from the properties list. 


\subsection{Open Assembly Model}

The reason to create an Open Assembly Model (OAM) [9] was to provide a standard representation and exchange protocol for assembly and system level tolerance information. The OAM structure was created to be extensible, and in the current UML version (see Figure 2), it is possible to store data for tolerance representation and propagation, representation of kinematics, and engineering analysis at the system level. The assembly information model focuses on the information requirements for part, features and assembly relationships. The data structure used is part of ISO 10303, informally known as the STandard for the Exchange of Product model data (STEP) [15] [16] [17] [18]. Information about assembly relationships and component compositions are incorporated in the schema. The convention utilized is the same as the CPM overview.

The class AssemblyAssociation represents the component assembly relationship of an assembly. It is the aggregation of one or more Artifact Associations.

The assembly relationships between one or more artifacts are represented by the class ArtifactAssociation. In most of the cases, two or more artifacts are involved in this relationship. However, the possibility of one artifact association in the OAM is also allowed to represent special cases. Such a case may occur when an artifact is to be fixed in space for anchoring the entire assembly with respect to the ground. It can also occur when kinematics information between an artifact and the ground is to be captured. Such cases can be regarded as relationships between the ground and an artifact. For these reasons the artifact association with one artifact associated is allowed. Please see [9] for the detailed description of the model. 


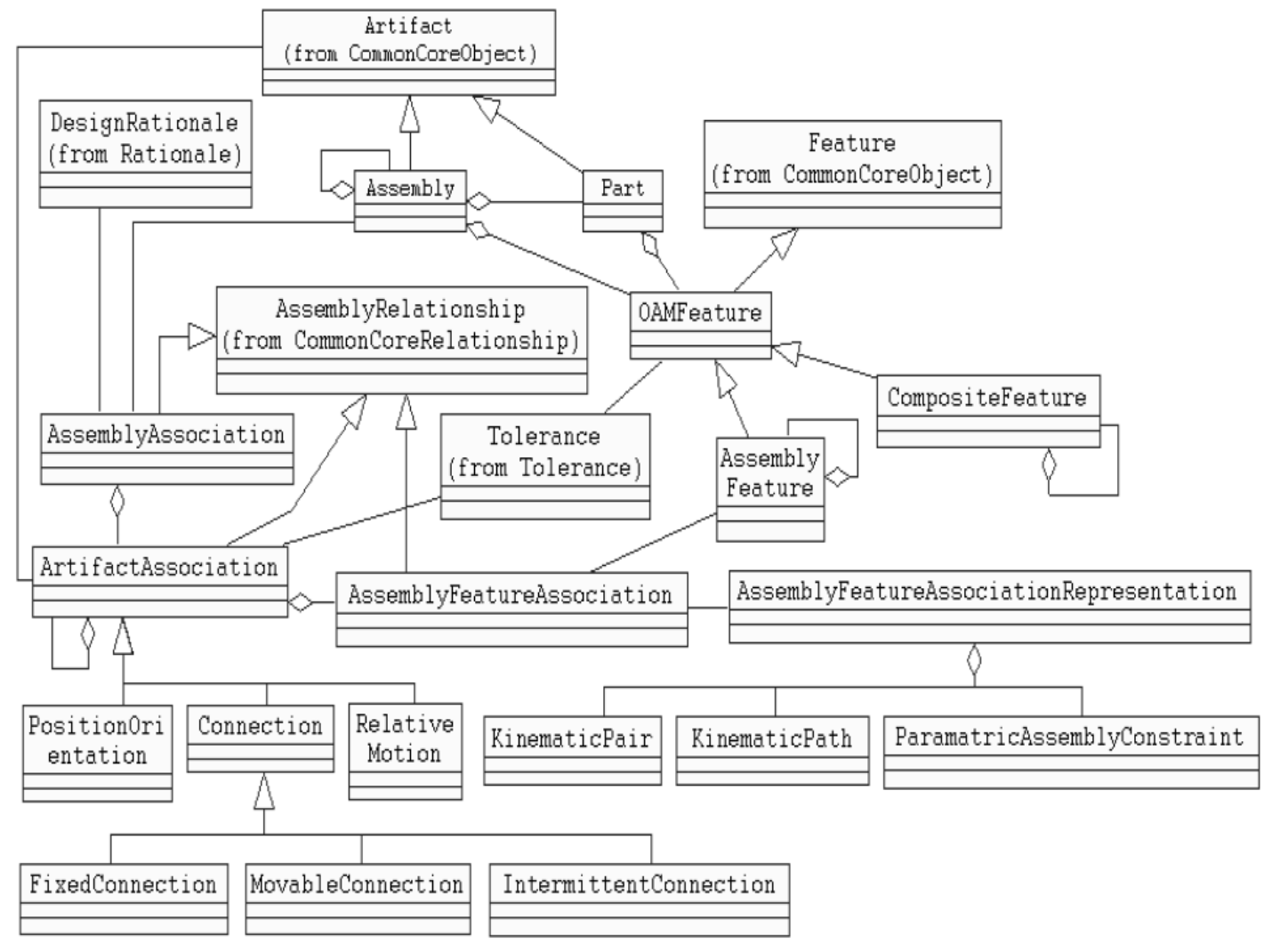

Figure 2 Overview of the OAM-UML

Based on the CPM/ OAM models discussed above, the motivation was to further explore and extend the current OAM with reasoning capabilities exploiting the ontological representation. We also intend to experiment with various tools for modeling an ontological assembly representation. Besides defining concepts, the benefits of such an ontology include [19] [20]:

- Creating abstract models

- Explicating concepts, properties, relations, functions, axioms and constrains

- Creating computer interpretable formulizations so as to infer classes, instances, or in general, reasoning through queries.

\section{Language concepts and tools}

In this section we first present the general concepts needed to explain the creation of the OAM ontology. The first step of the ontology development is the translation of the UML structure in OWL [21]. The idea of incremental modeling allows us to take advantage of the experience and knowledge gained from the earlier deliverable versions of the model. The key was to start with a simple implementation of a subset of the model and then progressively evolve towards a full model. 


\subsection{Patterns for Translation}

UML was originally used in software engineering and more specifically to support the software development. For this reason, the modeling elements offered by UML are substantially aligned with the needs of object-oriented programming and to some extent relational databases, which are also software. The current extensive use of UML is due to its intuitive graphic representation.

OWL was developed to support the semantic web [10] [21] [22] [23], as an extension of RDF [24] [25], and its core mission is to enable interoperability through semantic data representation. There are several underlying constraints to achieve harmonization between these two languages [26]. However particular attention is needed while translating the classes, where no widely accepted rules for creating or evaluating collections of classes currently exists [27] [28] [29].

In this work we adopt general repeatable solutions (termed patterns) to problems often occurring while mapping a model from UML to OWL. We specifically define a set of mapping solutions [30] [31] for the following:

1) problems concerning general UML properties

2) problems regarding various property constructs used to describe association relationships

\subsubsection{Property Translating Pattern}

There are two main considerations while translating UML properties into OWL elements (for example, object properties, data type properties, etc). First is the naming strategy for UML properties and the second is the UML property type mapping to OWL elements. First, UML properties are local to their owning classes and thus two classes may have properties with the same name. In this case, the properties have to be renamed when they are translated into OWL properties or OWL classes to avoid naming conflicts. Secondly, UML properties may be of primitive data types (data type provided by UML as building blocks) or of class types (constructs built ad hoc for a specific model). Here, if a property is intended to be of primitive data type, corresponding OWL properties and classes have to be created for the translation.

\section{- UML properties into OWL properties or OWL Classes}

An important consideration is to decide if UML properties should be translated into OWL properties or OWL classes. If a UML property represents a decomposable concept, it should be treated as an OWL class [32] instead of OWL property in the OWL model. Properties and attributes concepts are modeled as resources and can be further identified by their Universal Resource Identifiers (URI). If a new concept, such as an attribute type, is later introduced to denote the attribute value in an attribute, it can be attached to the attribute resource. 


\section{- $\quad$ Naming strategy and General Rules}

The Class-name-Property-name convention can be used to rename UML properties for most of the cases. The class name is the name of the class that owns the property. This class will be set as domain of the OWL property while the type of the UML property will be set as range of the OWL property. The following rules can determine which kind of OWL properties are used to translate UML properties:

1. If the property is of type class, use owl: ObjectProperty (property for which the value is an individual).

2. If the property type is a primitive data type, use owl:DatatypeProperty (property for which the value is a data literal, such as a string or a number): in this case the values of the property are treated as atomic types and cannot be decomposed further in conceptual modeling.

3. If the property has a multiplicity of 1, use owl: FunctionalProperty (a property that has a unique value $\mathrm{y}$ for each instance $\mathrm{x}$ ) or specify cardinality equal to 1 .

The drawback of this method is that all local properties must have unique names in an OWL document, which may result in generating a large number of properties. However, since there are only a handful of properties specified in CPM and OAM, this approach is adequate in most cases.

\section{- Using Inheritance}

Sometimes, making all local UML properties to be global properties in OWL may cause redundancy and naming conflicts. Inheritance of properties in OWL can be used to eliminate such problems. First, a unique generic property without specifying any classes as its domain (the class owning the property) and range (the class of the values of the property) type will have to be created. The following rules may apply:

1. Restriction on domain and range will be further specified only when the property is refined in a specific class description.

2. The generic property can be used in many different class descriptions. A unique name needs to be assigned to this property and restrictions will be applied to each single class.

The use of the inheritance property allows us to overcome the limitation of the absence of qualified cardinality restrictions, not supported in the OWL version 1.0. Consider the example in which there is a Class A connected with a property to $\mathbf{C l a s s} \mathbf{B}$, superclass of ClassC. To specifiy that an individual of ClassA has to be connected at least with two individuals of ClassC, we have to create a subproperty specifically connected with ClassC and then add the restriction on that subproperty. Moreover, to prevent the use of 
the superproperty connected to an individual of ClassC we can specify that the range of that superproperty is constituted by all the elements in ClassB that are not in ClassC.

Another use of inheritance property is when, for example, there is a ClassA connected with Class B and ClassC through two different properties (each class is range of each property). To specify that an individual of ClassA is connected with only one of these two properties, we have to assert that both the two properties are subproperties of a generic property, having as domain ClassA and having range not specified, and then add the cardinality restriction on this generic property.

\subsubsection{Association Pattern}

There are many different types of associations that can be described in UML, such as directed associations, binary associations, association classes, and so on. OWL also provides various property constructs to describe relationships. There are similarities and differences between these constructs. Both UML and OWL allow users to apply cardinality constraints, refinement, and sub-setting to associations and properties. However, UML supports n-ary relations (relations linking an individual to more than one individual or value), while OWL supports only binary relations. Besides, UML supports aggregation and composition relations between classes, while OWL supports transitive, symmetric, and functional property definitions. Association patterns are used to translate these UML association properties into OWL properties.

\section{- Simple directed association}

A simple directed association can be translated as an owl:ObjectProperty. The participating classes will be the domain and range of the associated property.

\section{- Classified binary association}

Associations in UML have various combinations of characteristics. For example, an association can be unnamed, shared, binary, and navigable. In mathematics, a binary relation (or a dyadic relation) is an arbitrary association of elements of one set with elements of another set.

A formal definition of a binary relation could be the following: A binary relation $R$ is usually defined as an ordered triple $(X, Y, G)$ where $X$ and $Y$ are arbitrary sets (or classes), and $G$ is a subset of the Cartesian product $X \times Y$. The sets $X$ and $Y$ are called the domain and range, respectively, of the relation, and $G$ is called its graph.

Analyzing this definition from the OWL point of view, it is easy to understand that $\mathrm{X}$ and $\mathrm{Y}$ are simply two classes of the ontology (e.g., Assembly and ArtifactAssociation) and that $\mathrm{G}$ is a property between them (e.g., Assembly2ArtifactAssociation). These properties are not shared between classes, unlike the ones previously presented.

In order to translate these binary associations into OWL, a taxonomy of binary associations is first created. The root of this taxonomy will be binaryAssocation. 
The subproperties of binaryAssocation include binaryUnnamedAssociation, binaryNamedAssociation, and associationclass. Since OWL properties are directional, for a given UML binary association a pair of mutually inverse properties is created. For example, two mutually inversed properties are created as subproperties of binaryUnnamedAssociation property. BUA_1 and BUA_1_INV are created as a pair of mutually inverse OWL ObjectProperties for further extension. The hasoutputFlow and isDestinationof properties can thus be organized as subproperties of BUA_1 and BUA_1_INV respectively. This pair of properties can be used to describe the association between Artifact and Flow (see section 2). Some ontology editors, such as ProtégéOWL, can take advantage of this arrangement and automatically generate the corresponding inverse subproperties.

\section{- Shared aggregation association}

The significance of the shared aggregation ${ }^{1}$ associations in UML is that the parts can be shared by many containers. Such semantics cannot directly be captured by OWL constructs. Similar to the classified binary association pattern, the root of the aggregation properties taxonomy will be first created after which a pair of directional subproperties of this root will capture the aggregation.

\section{- Self-referenced weak composition association}

The self-referenced weak composition association pattern is used in CPM to capture: decomposition hierarchies, part-of relationship, and containment hierarchies. A part-of relation can be defined as a transitive, irreflexive, and asymmetric relation. OWL currently only supports the transitive property. Without irreflexive construct, it is not possible for one to state that a part cannot be part of itself. Without asymmetric construct, two parts may contain each other. These unsupported properties (at the time of this report) will be available in OWL 1.1. As already mentioned in the UML version of the model, core classes are characterized by reflexive relationships but in OWL there are no primitives to represent such relationships. Hence the structure has to be reproduced in the ontology using a composite set of properties grouped under the super property composition (Figure 3 Protégé composition ).

\footnotetext{
${ }^{1}$ In some old UML documents, a shared aggregation is also called weak composition as opposed to the black diamond (strong) composition in which the containing component is responsible for the storage and creation of the contained components. Components in weak composition can be stored by more than one container.
} 


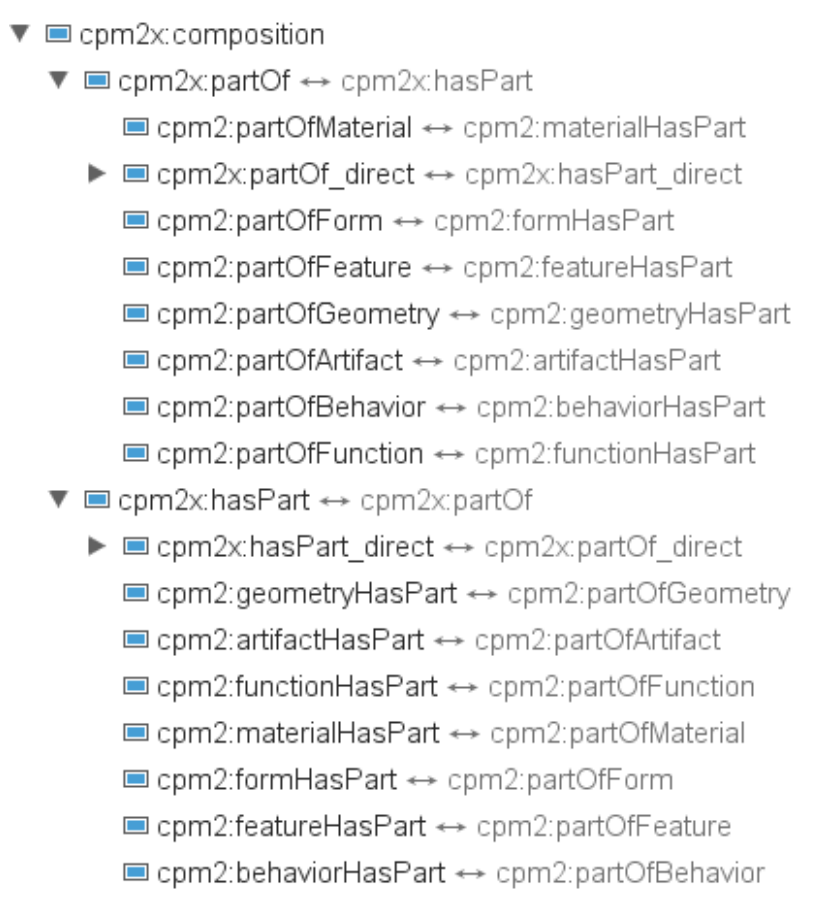

Figure 3 Protégé composition

As can be seen from the Figure 3 the class composition has two subproperties: hasPart and partof, each inverse of the other. Considering hasPart it can be noticed that it further has subproperties like hasPart_direct.

Note that the second properties have subproperties too. The subproperties of this class differ from the "not" direct properties because they are not transitive. For further explanation of the hierarchy let's consider a description of a bicycle and its decomposition into elements:

Bicycles have parts Wheel, DriveTrain

Wheels have parts Rim, Tire

DriveTrains have parts Gear, Chain

For expressing the part-whole relations [33] between individuals, we use hasValue with partof_direct and the relations between classes using the restrictions someValuesFrom with partof_direct. Following are some useful conclusions drawn from the above example: first of all the semantics necessary for the correct representation for bicycle parts are not completely represented by existential restrictions for e.g. owl: someValuesFrom. Considering the Chain class, we can deduce that a chain is part of at least a drive train, but we cannot deduce that a particular chain cannot be owned by more then one drive train. Adding a cardinality restriction (e.g., maxCardinality 1) on the property partof to the definition of chain will not solve the problem either. A chain is also a part of the bicycle where the drive train is a part. For 
this reason OWL-DL does not allow transitive properties to have any cardinality restrictions. The creation of the property partof_directly is useful for the introduction of restrictions in the definitions of these classes. A single chain cannot be a direct part of more then one drive train and a drive train cannot be part of more then a bicycle, so in these cases a cardinality restriction specified on the property partof_directly is needed. Specifying cardinality constraints helps to create a precise representation but there is a trade off between model accuracy and computational time needed by the Reasoner.

\section{- Association class pattern}

There are several approaches for translating association classes into OWL classes. Let's consider for example the following UML structure (Figure 4).

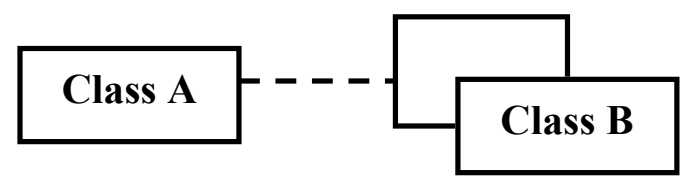

Figure 4 UML Property Class

In OWL it is not possible to represent this structure directly because there is no built-in pattern with this meaning. However, it is possible to decompose the UML structure into simple elements and later translate it in OWL to recreate the original meaning (Figure 5).

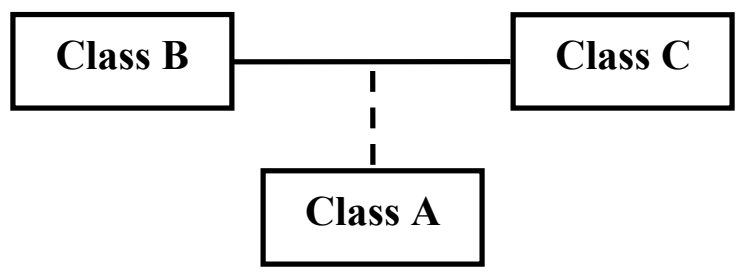

Figure 5 A Property Class connecting two different Object Classes

For the translation of UML association classes, in OWL there are two feasible solutions: the first requires the creation of a set of four different properties between classes with specific cardinality restrictions (Figure 6).

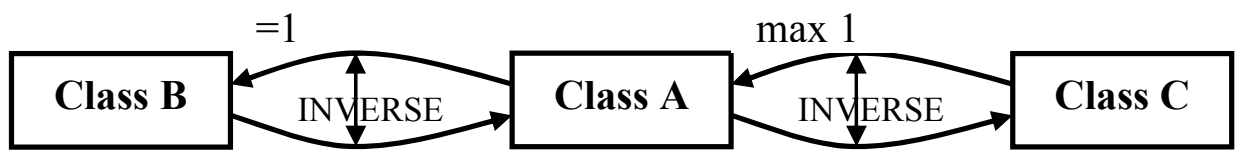

Figure 6 Property pattern for two Object Classes

This solution includes two different object classes, while in CPM and OAM this case never occurs. So we create an alternate second solution (Figure 7). 


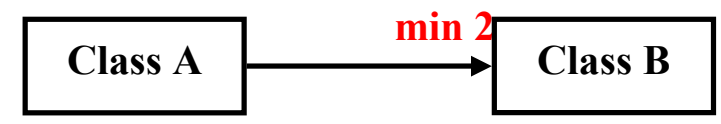

Figure 7 First part of the translation of Property Classes

The second solution is used with the specification of certain constraints. First of all (as seen from the Figure 7) a cardinality constraint has to be set and it is mandatory to preserve the binary relation between the two classes. This means that two instances of class B can be linked only with one instance of class A. In OWL, it is not possible to explicitly express this constraint but alternatively we declare that if the same two instances of class B are linked with two different instances of class A (A1 and A2) they have to be equal (Figure 8).
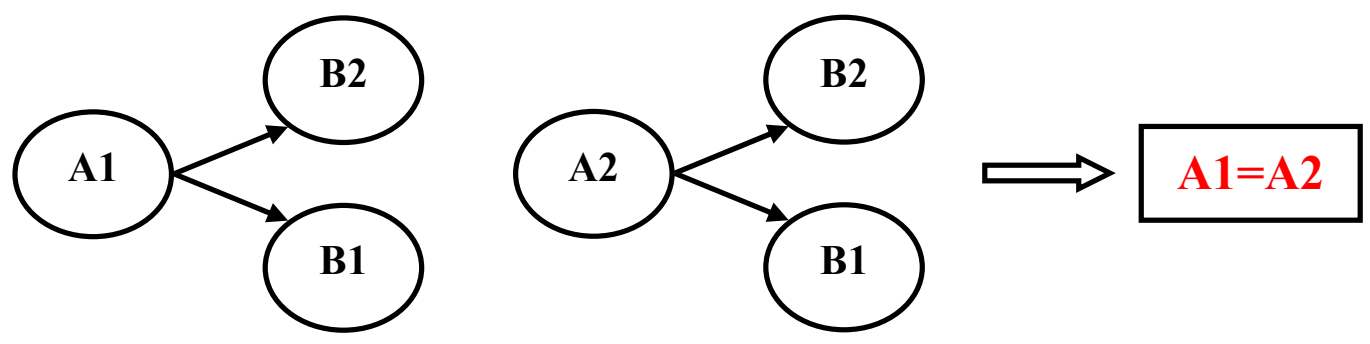

Figure 8 Second part of the translation of Property Classes

\section{- Cardinality Pattern}

All the above discussed patterns have to deal with the cardinality. By default, the cardinality restrictions for all properties in a model are set to be zero to many. Sometimes, it is hard to determine the cardinality of a relationship at an early stage of the design. Unlike UML, OWL allows redefining properties independently from the classes so we can specify cardinalities after the classes are defined. This provides more freedom to the model creators. However, caution has to be taken with the description-logic based approach since the changed cardinality may destroy the parent-child relations between the classes. Further examples will be presented later, when we discuss the strategies for using the CPM OWL model for various design phases.

\subsection{Modeling and Reasoning Tools}

The Assembly ontology is created starting from the core classes using the DL (Description Logic) sublanguage of OWL and Protégé-OWL to edit it. The preliminary modifications introduced in the model were tracked for later changes due to modeling necessities. A partial demo was created instantiating the model after every change or modification. We verified each branch of the ontology using Protégé-OWL, as it is able to run the reasoning simulation of the model. The Protégé-OWL editor [34] [35] is an extension of Protégé that supports OWL. Snapshots of Protégé Subclass explorer 
(Snapshot A1), Property browser (Snapshot A2) and Class browser (Snapshot A3) are presented in the Appendix.

The Protégé-OWL editor enables users to:

- Load and save OWL and RDF ontologies.

- Edit and visualize classes, properties and SWRL (Semantic Web Rule Language) rules.

- Define logical class characteristics as OWL expressions.

- Execute reasoners such as description logic classifiers [36] [37].

- Edit OWL individuals.

Although time consuming, this incremental approach (discussed earlier) of model development has allowed time for testing, and, subsequently improved the model.

Description logic reasoning are done using RACER [38], a DL reasoner. This tool allows consistency checking and to infer class/instances.

Rule-based reasoning is possible using SWRL [39] [40] rules. An appropriate plug-in was available in Protégé-OWL (SWRL Tab). A Jess Bridge [41] is used to translate both the rules and the ontology into the Jess Engine. Once executed, the results can be imported again into the ontology in OWL through the Jess Bridge.

\section{OWL Model of the Assembly}

One of the main benefits using an ontology is the possibility to share and reuse knowledge [42] [43] [44]. When importing one ontology from another, all the classes, properties and individual definitions that are part of the imported ontology become available for use and furthermore, we can add components or restrictions without affecting the imported ontology. In this research, since OAM is an extension of the CPM, we can first build the CPM ontology and then import it into the OAM ontology. With OAM, all the considerations about the CPM are still valid. Coherent with our aim to create a model that is extensible by itself, we choose to create a vertical hierarchy so that all the classes of the extensions will be subclasses of the main model classes. Section 4.1 presents the translation of the CPM to OWL and Section 4.2 subsequently presents the OAM translation. The reasoning in the developed OAM-OWL Assembly Model is presented in Section 4.3.

\subsection{Translation of the CPM to OWL}

The structure of the ontology begins from the class: CommonCoreEntity. This class represents real objects and relationships or associations between them. 
The common attributes type, name, and information for all CPM classes are defined for the CommonCoreEntity class. The first two are Datatypeproperties while the last is an ObjectProperty. The attribute type, set 0 or 1 , is useful to overcome the OWL drawback of not being able to set a class as abstract.

An abstract class is a class for which all instances are instances of a subclass. Such classes normally are used as base classes in inheritance hierarchies. In our case, abstract classes constitute the top few levels of the hierarchy. To determine whether a class is abstract or concrete we control the usage of type by manually setting the cardinality to zero for CommonCoreEntity's abstract classes and maximum cardinality back to one for concrete subclasses.

The name attribute can be completely ignored in the OWL document because rdfs:label and the URI for each resource can be used to achieve the same identification purpose. However, to preserve the semantics of CPM, the corresponding property commonCoreEntityName is created.

The information class is set like a class and not a DatatypeProperty (attributes) to allow users to define them with flexibility, for example to connect every object to any number of information. It has description, documentation, and properties attributes. The description and documentation can be represented by OWL DatatypeProperties for their values are the URI pointing to the referenced documents.

The properties attribute is a set of attribute-value pairs stored as strings representing all domain or object-specific attributes. It should be noted that the attribute-value pair may be extended to be attribute-type-value pair at the detail design phase. Unlike the other two attributes which are defined as DatatypeProperties, the set concept defined in CPM report has to be preserved for all these string values. To achieve this, properties and its attributes are treated as OWL classes.

The two subclasses of CommonCoreEntity are CommonCoreObject and CommonCoreRelationship. They are represented respectively in UML as two main groups of object classes and association classes. For this reason they have type set to 0 (because they are on a high level of the hierarchy) and they are connected with each other with the ObjectProperty property2class and its inverse, following the binary pattern of the association classes.

CommonCoreObject is the parent of five subclasses of which three are concrete classes (with type 1) and two are abstract. The former are Behavior, Requirement and Specification. They have the same type of connections as in UML, for example the Requirement and Specification will be joined by a relationship, and Behavior will have a self-reference relationship with the composition pattern. In this way the super-property Composition is divided into the subproperties partof and hasPart, composed of specific properties that connect Behavior with itself (both of them are transitive). 
Similarly, as subclasses of partof_direct and hasPart_direct we have partofBehavior_direct and behaviorHasPart_direct.

Two other subclasses of CommonCoreObjet are CoreEntity and CoreProperty. These are important classes because they are particularly involved in the construction of the OAM ontology and because they are connected with subclasses of CommonCoreRelationship (see Figure 1). CoreEntity has a binary relationship with EntityAssociation (coreEntity2entityAssociation) while CoreProperty has one with Constraint (coreProperty2Constraint).

Rationale is an attribute of CoreProperty with specializations (Flow, Form, Function, Geometry, and Material) and connected to the Requirement through a binary ObjectProperty.

The rest of the model is developed following the patterns previously described, paying attention to the meaning of the UML relationships and their cardinalities. However, it is necessary to underline the role of the classes Artifact and Feature as they are the main classes of the OAM OWL.

Further, we decided to preserve the UML interpretation of the relationship partof between Artifact and itself, so as to describe the composition of an assembly at the CPM level. This information can be useful for the core description of an artifact because some characteristics of an assembly can be influenced by the characteristics of its constituent parts (for example the function of a part partakes in the function of the assembly).

\subsection{Translation of OAM to OWL}

Starting from the CPM-OWL model (discussed earlier) a relative OAM ontology is built. In OAM all the classes and properties presented in section 4.1 are valid. In the following section we further discuss the translation of the OAM from UML to OWL.

\subsubsection{Relationships between artifacts}

First and foremost, for the translation it is important to represent the relationships between artifacts. Consider the following: $\operatorname{artifact} \mathrm{A}$ is composed by artifact $\mathrm{B}$, artifact $\mathrm{C}$ and artifact $\mathrm{D}$ as in Figure 9.

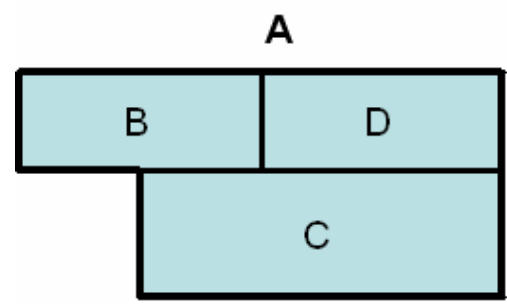

Figure $9 \mathrm{~A}$ is composed by $\mathrm{B}, \mathrm{C}$ and $\mathrm{D}$ 
There are different levels of decomposition for this product. Now consider artifact B composed by artifact $\mathrm{E}$ and artifact $\mathrm{F}$ (Figure 10).

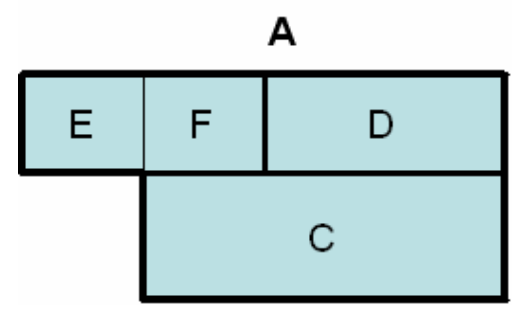

Figure $10 \mathrm{~B}$ is an assembly too

Here, we can have different relationships between products, for example artifact A can be composed by integrating artifacts $\mathrm{B}, \mathrm{C}$ and $\mathrm{D}$ or it can be created by the relationship of artifact $\mathrm{C}$ with $\mathrm{B}$ and artifact $\mathrm{C}$ with $\mathrm{D}$ (Figure 11) considering $\mathrm{B}$ and $\mathrm{D}$ not connected.

A

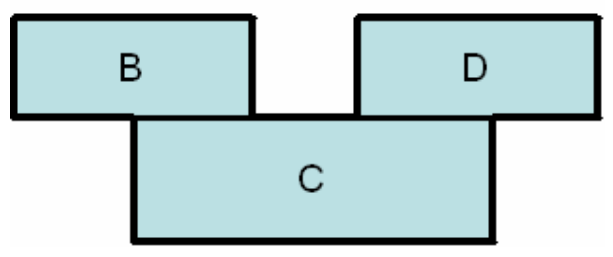

Figure $11 \mathrm{~B}$ and $\mathrm{D}$ are not directly connected

In the above example, we have to distinguish between artifacts composed by other artifacts (A and B) and artifacts that represent the leaf nodes of the composition (C, D, E and F). We classify them into Assembly and Part respectively (as subclasses of Artifact). For the Part class we associate the necessary and sufficient conditions such that a leaf node has cardinality 0 with the property artifactHasPart_direct that is inherited but not required. In this way, we define a part like an artifact without subassemblies, so with neither the direct nor the indirect properties artifactHasPart.

Contrarily, considering the necessary conditions of Assembly, there is a constraint to have at least two artifacts connected through the property artifactHasPart_direct (inherited from Artifact and hence not repeated for Assembly). By defining Assembly and Part like partitions of the class Artifact, an Artifact composed by other artifacts will be inferred to be an assembly. Unfortunately it is impossible to infer the opposite, which means that we have no way to assert that an artifact without direct artifacts is a part. This is one of the biggest limitations of ontologies in general. The logic is as follows: not relating an artifact with other subartifacts does not mean that this artifact is without subartifacts, it just means that at the moment we do not know if it will be composed by other parts. Although, it does have the relationship artifactHasPart_direct, but we do not know to which subartifacts it is connected. 
While populating the ontology the user will choose a level by level connection of the artifacts. This implies that he/she will decide on the link between them to instantiate the direct properties (artifactHasPart_direct or partofArtifact_direct). Here, there could be three choices while implementing the logic of the ontology, in particular choosing the connection with indirect properties. Let us take the example in which assembly $\mathrm{A}$ is composed by assembly $\mathrm{B}$ and assembly $\mathrm{C}$, and assembly $\mathrm{B}$ is made by part 1 and part 2 and assembly $\mathrm{C}$ by assembly $\mathrm{D}$ and part 3, assembly $\mathrm{D}$ by part 4 and part 5 (this is the composition of the direct properties shown in Figure 12).

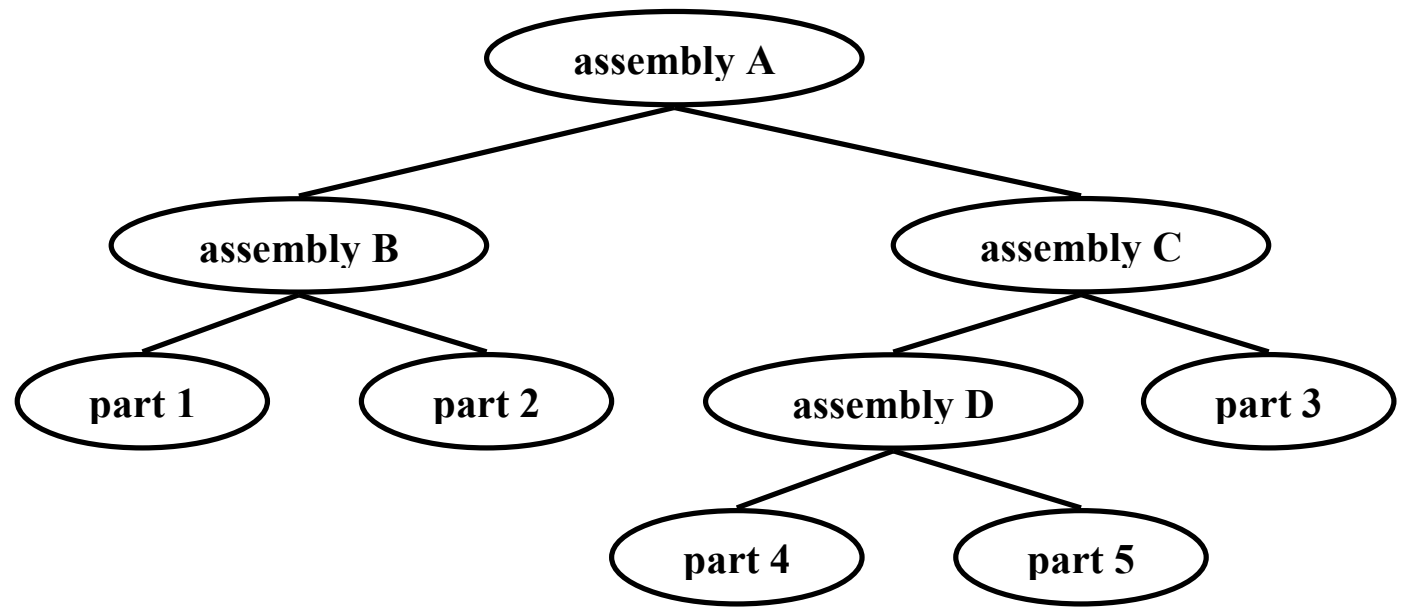

Figure 12 Example of an assembly composition

The three different choices of inferred connection to the assembly A through the indirect property artifactHasPart are:

- part 1, part 2, part 3, part 4, part 5, assembly B, assembly C and assembly D: all the levels

- part 1, part 2, part 3, part 4 and part 5: only parts at all levels

- part 1, part 2, part 3 and assembly D: the second level of the decomposition

We choose the second solution because it is less confusing than the first and more detailed than the third. So, if we want to see the detailed composition of the assembly it is sufficient to check the direct property of each subassembly and if we want to know the parts that compose the assembly we can check the indirect properties. Moreover, since the user has to choose only the direct property, we have to decide to allow or not, the possibility to create an assembly with two subassemblies without specifying their constituent parts. Here, the question arises as to if there is any meaning to define an assembly without describing the parts by which it is composed? Or does an assembly exist without parts?

Although from the definition the lowest level of the tree has to be represented by parts, we choose to define an assembly even without specifying the constituent parts. The reason is as follows: during the concept phase of the product life cycle we have to allow the representation of an assembly without specifying its composition. For example we 
may represent a car simply made from an engine and 4 wheels, without specifying from which parts the engine and the wheels are composed.

So, in this ontology, there are two approaches: the bottom up, useful when we want to structure some data with complete information, and the top down, necessary in cases when we do not know the exact composition of the assembly but we have just an idea of its organization.

After choosing the rules to represent relationships between an assembly and its constituents, the second step is the representation of relationships between these constituents. In the UML model, we have two classes for representing the structure of the assembly: ArtifactAssociation, which represents the relationship between subassemblies and AssemblyAssociation, which is the collection of the elements of ArtifactAssociation.

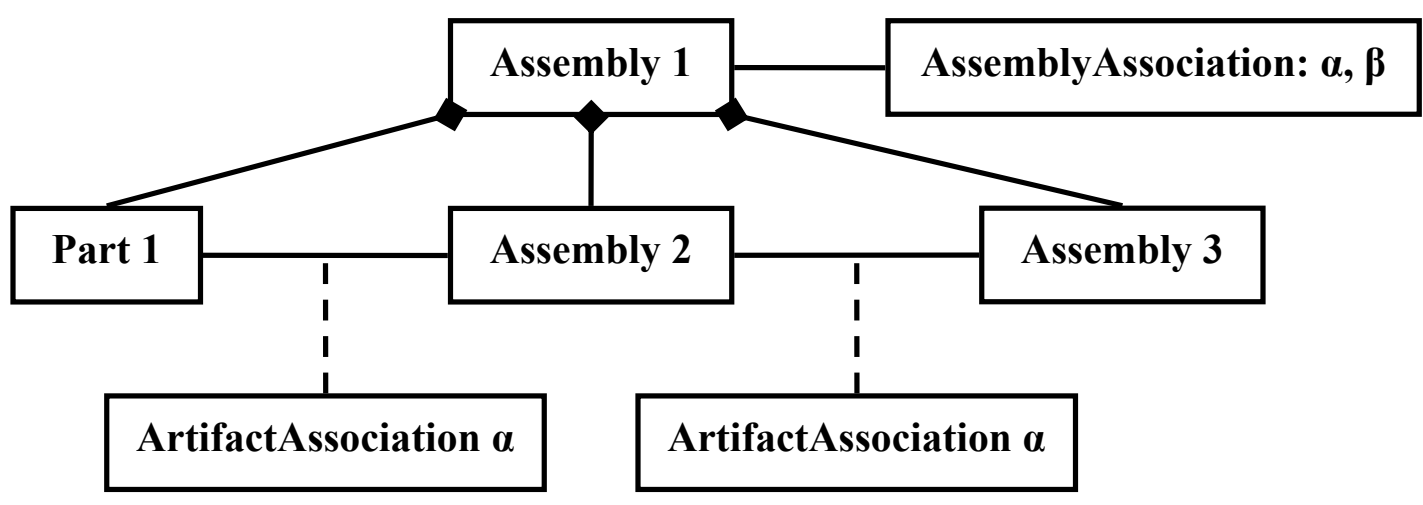

Figure 13 The assembly representation in OWL

In the ontology the class ArtifactAssociation is created with two different ObjectProperties connected to it: the first one (artifactAssociation2Part) relates the constituents of the assembly and is a specification of the binary association entityAssociation2CoreEntity while the second one (artifactAssociation2Assembly) is the property that connects ArtifactAssociation to the Assembly.

In this way the UML class AssemblyAssociation is replaced by the property that directly connects the elements of ArtifactAssociation with the assembly. For example, if assembly $\mathrm{A}$ is composed by part 1 and part 2, there will be an instance of ArtifactAssociation that connects part 1 and part 2 through the property artifactAssociation2Part (ArtifactAssociation has to be connected with at least two instances of Parts or Assembly). The same instance of ArtifactAssociation will be connected with the assembly A through the property artifactAssociation2Assembly (assembly A has to be connected with at least one instance of ArtifactAssociation). 


\subsubsection{Relationships between features}

Although the information regarding the assembly composition is useful to give an overview of an artifact, we need to detail the representation to understand in which way parts are connected together and the positions and profiles interested in the assembly.

In the CPM we have already represented the relationship between an artifact and its features through the ObjectProperty artifactHasFeature. Our aim in OAM is to represent that a feature, although still remaining characteristic of a part, will meet another feature belonging to another part to form the assembly. We term this particular feature OAMFeature, subclass of Feature. We include in the OAM the possibility to connect these OAM features together, and so as to connect the parts that have these features and finally to create the assembly in the ontology.

The class that represents the link between two or more features is termed AssemblyFeatureAssociation (AFA) (subclass of EntityAssociation). It is connected to the features through the property AFA2Feature following the property class pattern. Once this property is defined, we give a formal definition (necessary and sufficient condition) to the OAMFeature as a feature with at least one connection with the class AssemblyFeatureAssociation.

Like in the UML model, it is useful to have a direct connection between the class that represents the association between features (AFA) and the one that represents the link between the artifacts that own these features (ArtifactAssociation). For this, we use an ObjectProperty that is a binary property but not a subproperty of property2class, since it connects what in UML were two property classes. This property is called artifactAssociation2AssemblyFeatureAssociation (its inverse is called assemblyFeatureAssociation2ArtifactAssociation). Logically, an element of AssemblyFeatureAssociation can participate only in one ArtifactAssociation while an instance of ArtifactAssociation can be represented by one or more associations of features.

\subsubsection{Pairs and Relations}

So far, we can describe an assembly through the parts and the features that are involved in it. Here we need to detail the description of an assembly with a section relative to pairs existing between features. Besides we also need to know if they are movable or fixed, the kind of constraints between them and so on.

In the ontology we choose, like in the UML version, to leave a single class that reunites this information: AssemblyFeatureAssociationRepresentation (or AFAR). This is still an EntityAssociation because it represents the relationship between two or more features. It will have the same restrictions developed for the property class pattern and will be connected with the AFA to which it refers: the AFAR will link together the same features that are involved in the AFA. Moreover, the AFA has to have just one AFAR 
linked with it, since AFAR is by itself the class that clusters all the information about the connection. This information will be represented through the same classes as in the UML model: ParametricAssemblyConstraint, KinematicPair and KinematicPath. They are all again subclasses of EntityAssociation, they have the same children classes as in the UML version and additionally they refer to the same features involved in the pair.

The properties connecting AFAR to these three classes are aggregations and they are collected under a unique property called AFAR_details. In the ontology an association between features can have utmost one path and one pair while the number of constraints is flexible.

Consider the class KinematicPair which represents the kinematic constraints between two adjacent features at a joint: to be defined it needs to be connected with the classes PairRange, PairValue and PairFrame. PairRange specifies the allowable configuration range of the two features in the form of upper and lower bounds. PairValue specifies the current configuration (value) of the two links between the two bounds. PairFrame represents a coordinate system attached to a feature. A kinematic pair needs two coordinate systems to describe its kinematic behaviour as to where they will be attached to the two relevant features. For the pairs, the properties involved are grouped into one property that connects the pair with its range, value and frames, and another property is used to connect these frames to the reference features.

The class KinematicPath provides the description of kinematic motion. It is the aggregation of path elements along which the motion is to take place. A PathElement can specify different types of paths. Since the KinematicPath is composed of a set of PathElements, it can describe a composite path as well as a simple path. To connect the PathElements a composite class PathElementConnection is used to order the elements by defining the precedence. For this reason there exist the properties isNextElementof and isPreviousElementof.

PathElement is a path segment with two PathNodes, which represent the "from" node and "to" node, respectively. Two different properties are established going from the PathElement to the PathNodes, both with cardinality 1. PathNode is used to define the start and end locations of a path. At each PathNode, the position and rotation of a frame along the path need to be defined.

\subsubsection{Tolerances}

Like in the UML OAM, we introduce tolerances in the model. We want to allow for design tolerances from the early stages of the product lifecycle, to combine the tolerances definition with the assembly structure [45]. A proactive approach could be useful for an early tolerance synthesis and analysis when the design is incomplete.

The class Tolerance has the same subclass structure of the UML version, i.e., geometric and dimensional tolerance. Tolerance is connected with OAMFeature to represent tolerances important in the pairs of parts/subassemblies. The choice of tolerances is 
particularly difficult when different parts have to be combined together to obtain a specific functionality.

DimensionalTolerance is linked to the class Size because it controls the variability of linear dimensions. Size is a subclass of Attribute, so it is connected with all the CommonCoreEntities, including features. The GeometricTolerance defines the allowable variation for the form, size of individual features, allowable variation in orientation and location between features. For this reason, this class is connected to Geometry (a subclass of the CPM class CoreProperty connected with the form of an artifact or of a feature). Of the geometric tolerances we can underline important ones: LocationTolerance, OrientationTolerance and RunoutTolerance. Unlike other tolerances they are also linked with the class Datum. This class represents the geometries that are chosen like a reference for the tolerance. So here, Datum is a subclass of Geometry and it is connected with DatumFeature that is a subclass of OAMFeature. The individuality of the datum is that it can be described like a particular geometry, for example like a point, a curve or a plane. Now that we already have these elements in the ontology, we exploit the potentiality of OWL to build a class that is a subclass of different classes (multiple inheritance). For example, the class CurveDatum will not only be a subclass of Datum but also a subclass of Curve. In this way it inherits the properties and the characteristics of both Curve and Datum. The practical way to realize this is to define the two conditions in the specification of the class.

\subsubsection{Usage Patterns}

The next task is to improve the model since it requires redundant specification of the subassemblies, parts, and artifact associations used more than once. For example, if a wheel subassembly in a car is comprised of a tire and a hub, this subassembly must be repeated at least two times in the current model, so that each wheel subassembly could be attached to the correct axle. Otherwise, there is no way to distinguish the back wheels from the front ones.

This leads to a number of problems:

- Consistency maintenance is difficult when a reused subassembly is changed, and requires propagation to all its usages. For example, there is no central class to make a change to the parts of wheels, and then propagate this change to all assemblies using wheels.

- Finding all the usages of a part or subassembly is unreliable. For example, the only information available about location of wheels usage is in the names "FrontWheel" and "BackWheel," which may change over time.

Hence it is important to find a central class that represents the elements to be used more than once and then to create some classes to realize the usages of these elements. This problem concerns not only the elements of the assembly but also the relationships in this assembly. 
The general pattern is that for any element of an assembly, we define a corresponding usage element that refers to the original element and the context of its use.

The elements that can be repeated (used more than once) are Artifact, OAMfeature, ArtifactAssociation and AssemblyFeatureAssociation: these are the Reusable elements (Figure 14). For each one of them we define a corresponding class Usage, so in the model we have the following classes: ArtifactUsage, AssemblyFeatureUsage, ArtifactAssociationUsage and AssemblyFeatureAssociationUsage. Every concept that is to be repeated will also have a particular Context. This means that Artifact and ArtifactAssociation will be repeated within an Assembly (the context of these classes) while in the context of ArtifactUsage we can find several OAMfeatures and AssemblyFeatureAssociations.

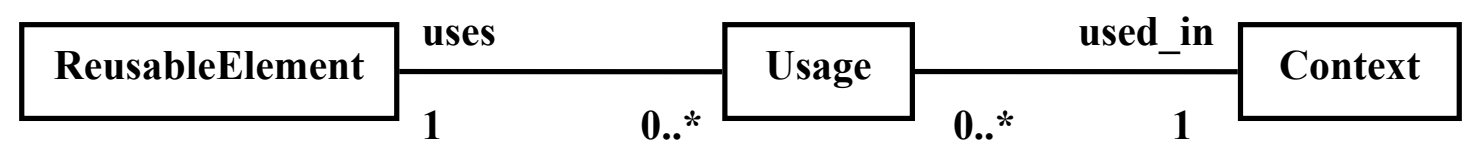

\begin{tabular}{|l|l|l|}
\hline ReusableElement & Usage & Context \\
\hline Artifact & ArtifactUsage & Assembly \\
\hline AssemblyFeature & AssemblyFeatureUsage & ArtifactUsage \\
\hline ArtifactAssociation & ArtifactAssociationUsage & Assembly \\
\hline AssemblyFeatureAssociation & AssemblyFeatureAssociationUsage & ArtifactUsage \\
\hline
\end{tabular}

Figure 14 Usage pattern

The property uses will connect every Usage to its corresponding ReusableElement. The cardinality will be equal to 1 because each repeated element can belong to only one element. The inverse of this property is isUsedBy and has free cardinality because every element can either follow the usage pattern or not and can have more than one repetition. Usage is connected with the context through the property used_in. The cardinality 1 on this property constrains the Usage to have not only its referring element but also its context. The property hasUsage is used to link contexts with usages and it is declared as the inverse of used_in.

Let us take into consideration a composition of an assembly formed by repeated parts or subassemblies. This assembly will be connected through the hasUsage property with the ArtifactUsage that represent the repetitions and with the ArtifactAssociationUsage that represents the repeated association between these repetitions. Every ArtifactUsage will be referred to its corresponding element through the property uses that explains which element is repeated. The same holds for the ArtifactAssociationUsage. 
In the example (see Figure 15) of the car we will have a representation similar to the above discussion.

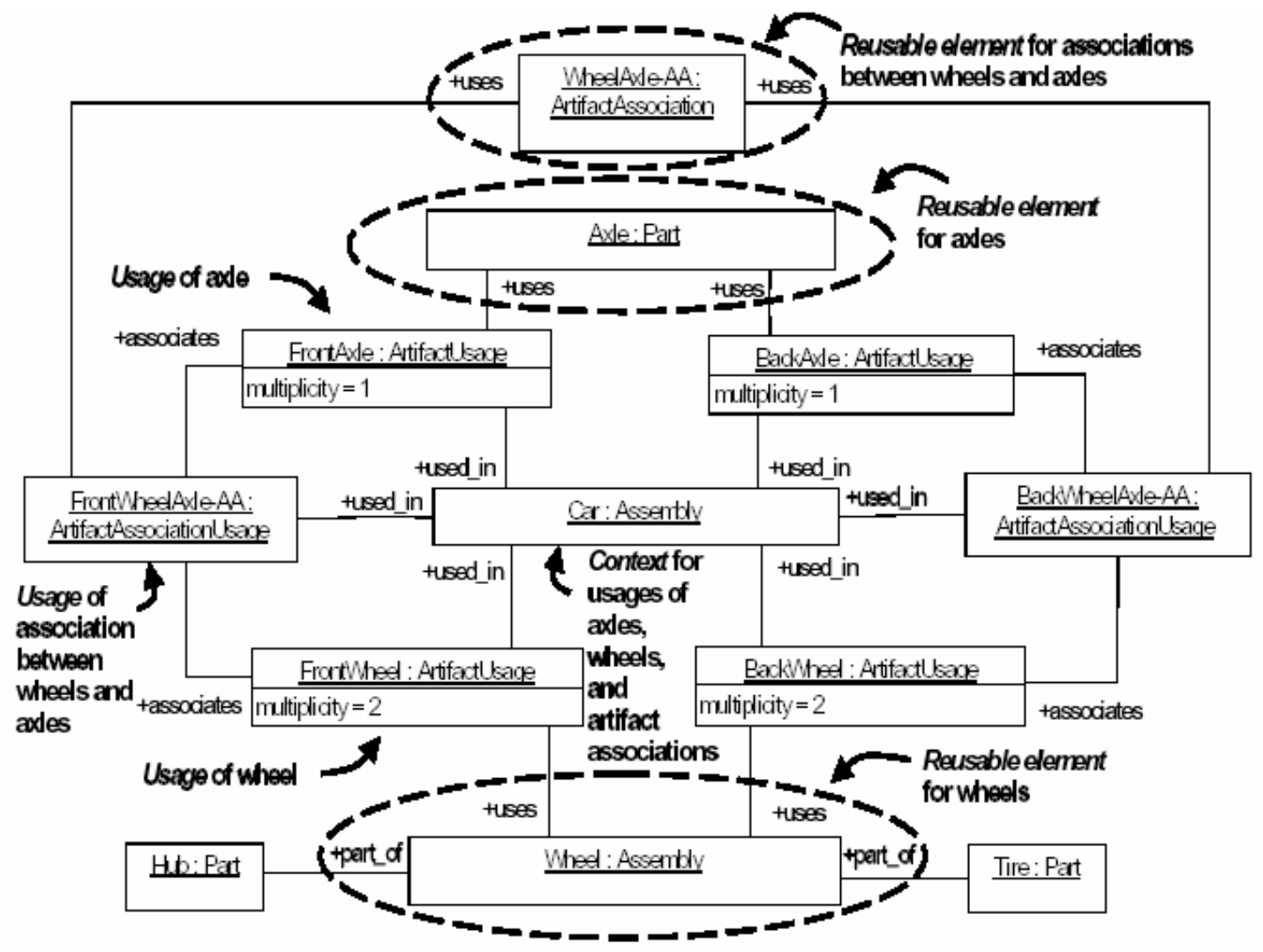

Figure 15 Example of the usage pattern

The most important element in representing this usage pattern is its implementation and its advantages in using an ontology instead of a UML model. In fact in OWL we can create a class that is a subclass of more than one class so that it inherits the properties and the characteristic of every parent class. In the same way, one instance can instantiate more than one class, taking all the properties of these classes. The coherence control will enable us to verify the correctness of such assertions. These OWL characteristics are exploited in the usage pattern because the model setting will be the same for both cases when we do or do not use the usage pattern. The instantiation of the model will follow the steps for which we define the Artifacts:

- we can either directly connect the Artifacts through the hasPart_direct, isPartof_direct when we don't need to use the usage pattern, or

- we can connect them with their repetition elements (ArtifactUsage) if we want to use the usage pattern.

This is allowed because an Artifact will have the possibility to have subassemblies or to be a subassembly and at the same time to belong to the ReusableElement class having property isUsedBy. If we want to choose to follow the usage pattern, we have to 
employ some SWRL rules. Without them, for example, the ArtifactAssociation will not be connected to any Artifact but only with the ArtifactAssociationUsage. This will be presented as an inconsistency since there is a restriction that an ArtifactAssociation has to link at least two Artifacts. For this reason we have built some rules (explained in the following section) to automatically compile the field artifactAssociation2Artifact property. The same kind of reasoning will be followed for each usage concept.

\section{Reasoning with OWL Assembly Model}

Classes, properties and restrictions are the elements that OWL offers for the creation of an ontology but these elements are not sufficient to capitalize the real potentials of an ontology, i.e., the reasoning capabilities. In this ontology we can execute two different kinds of reasoning, the description logic reasoning using RACER and rule-based reasoning using SWRL and Jess.

\subsection{Description Logic Reasoning}

Description logic reasoning is done using RACER, a software that works like a reasoner. RACER practically exploits all the restrictions and the definitions of the classes to infer classes and instances. If for example, we define a Class A and we specify a necessary and sufficient restriction on its properties (i.e., a pizza is defined like a food that has a base and some toppings) and we separately define a Class $\mathbf{C}$ that has its properties specified (Margerita pizza is a food that has a base and has, as toppings, cheese and tomatoes) the reasoner can infer that Class $\mathbf{C}$ is a subclass of Class $\mathbf{A}$ (i.e., Margerita is a pizza with additional characteristics). In the same way the reasoner can infer instances. By always using the necessary and sufficient conditions RACER can associate an instance to a different class if it satisfies these conditions. Taking the previous example, it means that if we define Margerita as a pizza that has only cheese and tomatoes as toppings and we create an instance of Pizza that has only these two ingredients, the reasoner will infer that this instance belongs to the class Margerita. The role of the reasoner is also to check the consistency of the ontology by verifying the necessary conditions and the tree of the classes.

\subsection{Rule-based Reasoning}

Since the description logic reasoning cannot be applied to properties, we chose to apply some SWRL rules (interpreting the ontology through a Jess Bridge) to improve the reasoning capability of the ontology. Once the Jess Engine is run, it returns the new inferred information to the ontology. In the subsequent paragraphs we will first explain the rules in general, and then discuss every specific rule. There are 4 kinds of rules that are useful both to associate instances to new classes and to create properties between instances. 


\subsubsection{Property rules}

Property rules are the most common in the ontology, they are used to create new links between instances once some properties are satisfied (Figure 16). They are logic rules and they incorporate the meaning into the ontology. They are useful because, when defining the ontology, we do not need to specify every property: doing so we can avoid mistakes and thus input a lighter ontology. For example, once we have the structure of an Assembly and the ArtifactAssociations between its subassembly, the Jess Engine can link these ArtifactAssociations to the Assembly. In the example of Figure 16 the Assembly 1 is composed by Part 1 and Part 2 (property partofArtifact_direct), these last are connected through the ArtifactAssociation $\alpha$ (property artifactAssociation2Part): the Jess Engine infers the connection between $\begin{array}{lllll}\text { Assembly } 1 & \text { and } & \text { ArtifactAssociation } & \text { (property }\end{array}$ artifactAssociation2Assembly).

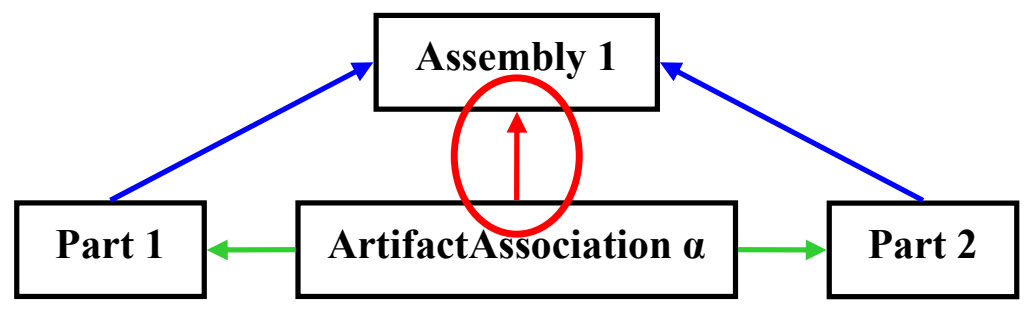

Figure 16 Property rules

\subsubsection{Association rules}

As mentioned earlier in this report (section 3.1.2), we have decided to uniquely translate the property classes and the relationships they specify between two elements of the same object class. The property class will become a normal class in the ontology and it will be connected to the object class with a binary property. To be binary, first of all we apply a minimum cardinality 2 , and then we specify that if two different elements of the property class are connected to the same elements of the object class, then these two elements are the same. In SWRL it is translated with the embedded language structure: sameAs.

\subsubsection{PartOf rules}

These rules (see Figure 17) are needed to infer the indirect properties (discussed earlier in the structure of Assembly). The user only specifies the properties partofArtifact_direct while the partofArtifact will be built through SWRL rules. 

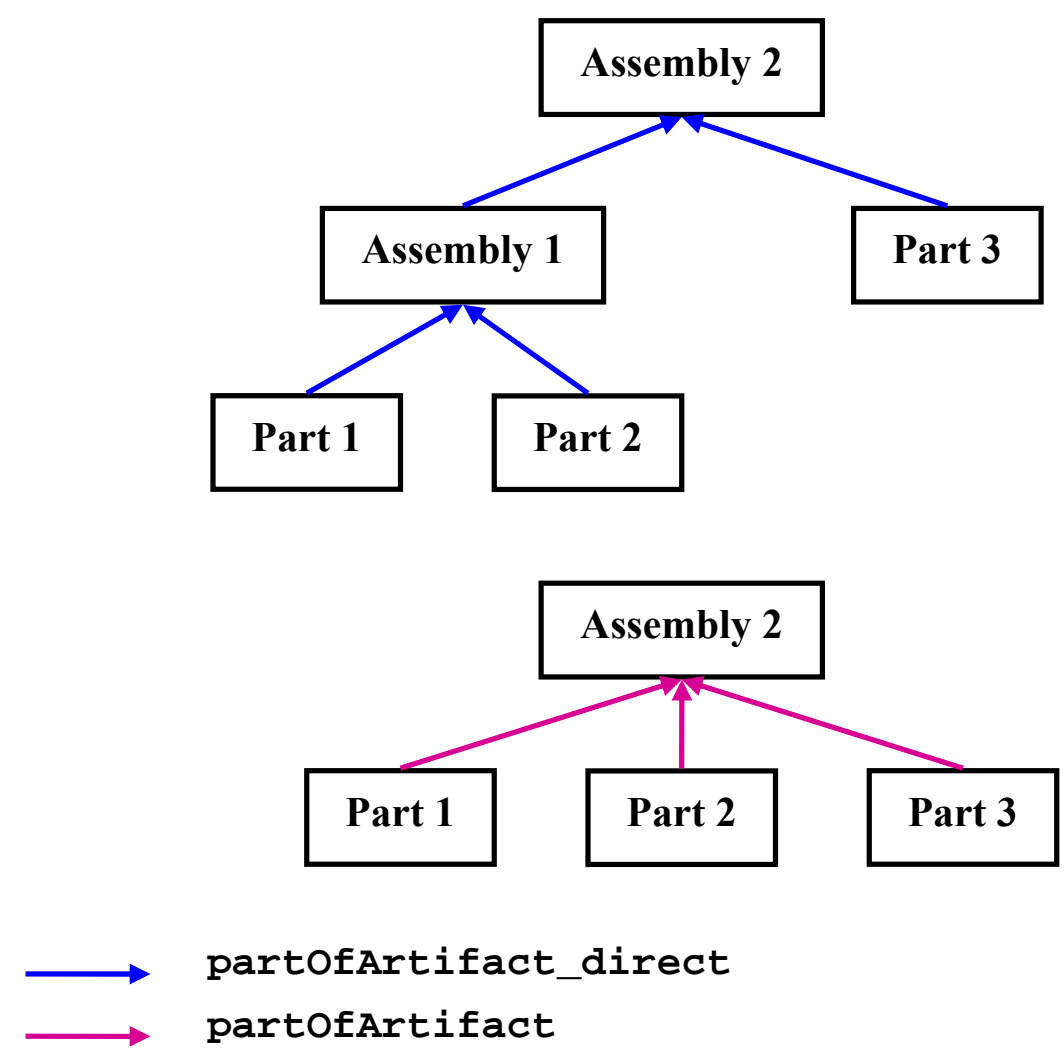

Figure 17 PartOf rule

\subsubsection{Restrict rules}

They are useful to populate the classes of the kind not-allowed. We have to create these classes because in OWL, and in particular in Protègè, there is no way to infer that some impossible properties or instances created by the user have to be cancelled from the ontology. The only way to realize this is to insert the user's input without meaning into new classes. Take for example the case in which an assembly is composed by itself (i.e., assembly 2 is composed by assembly 1 that is in turn composed by assembly 2 ) as can be seen from Figure 18.

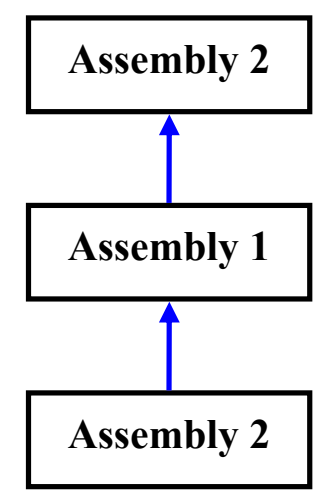

Figure 18 Example of a not allowed assembly 
Some traditional SWRL rules or some OWL definitions are useless because they can only specify some characteristics of the classes but not of the instances. In the example, both the assembly 2 and 1 are instances of the same class, so no definitions can be applied to the relationships between them. To solve such problems we create the not-allowed classes and we put such structures in them through SWRL rules.

\subsection{Rule Analysis and Discussion}

In this section we examine the rules in the Ontology. Note that we sometimes may need to present some of them together, because they are useful only if they are run together. The rules will be presented in tables with the antecedent in one column and the consequent in the other (similar to the structure if-then in other languages). The first group of rules we analyze is useful to create the relationship between an assembly and the ArtifactAssociations it is composed of.

Table 1 presents the ArtifactAssociation rules. We need four different rules because an ArtifactAssociation can connect both parts and assemblies. As already mentioned, we allow this because usually in the first stage of the product lifecycle we need to describe an assembly in general, without considering the individual parts by which it is composed. However in the manufacturing phase, we specifically need to describe the assembly. We want to allow both of these situations, but the drawback is the lack of information as to whether or not there will be a complete description of the subassemblies.

\begin{tabular}{|c|l|l|}
\hline Rule & \multicolumn{1}{|c|}{ Antecedent } & \multicolumn{1}{|c|}{ Consequent } \\
\hline 1 & $\begin{array}{l}\text { artifactHasPart_direct(?x, ?y) } \\
\text { Part(?y) } \\
\text { artifactHasPart(?x, ?z) } \\
\text { Part(?z) } \\
\text { differentFrom(?y, ?z) } \\
\text { part2ArtifactAssociation(?y, ?a) } \\
\text { part2ArtifactAssociation(?z, ?a) }\end{array}$ & \\
\hline & $\begin{array}{l}\text { artifactHasPart_direct(?x, ?y) } \\
\text { Assembly(?y) }\end{array}$ & \\
& $\begin{array}{l}\text { artifactHasPart_direct(?x, ?z) } \\
\text { Assembly(?z) } \\
\text { differentFrom(?y, ?z) }\end{array}$ & \\
2 & $\begin{array}{l}\text { artifactHasPart(?y, ?q) } \\
\text { Part(?q) } \\
\text { artifactHasPart(?z, ?r) } \\
\text { Part(?r) } \\
\text { differentFrom(?q, ?r) } \\
\text { part2ArtifactAssociation(?q, ?a) } \\
\text { part2ArtifactAssociation(?r, ?a) }\end{array}$ & \\
\hline & $\begin{array}{l}\text { artifactHasPart_direct(?x, ?y) } \\
\text { Assembly(?y) } \\
\text { artifactHasPart_direct(?x, ?z) }\end{array}$ & Assembly2ArtifactAssociation(?x, ?a) \\
3 & $\begin{array}{l}\text { Assembly(?x) } \\
\text { part2ArtifactAssociation(?y, ?a) } \\
\text { part2ArtifactAssociation(?z, ?a) }\end{array}$ & \\
\hline 4 & artifactHasPart_direct(?x, ?y) & Assembly2ArtifactAssociation(?x, ?a) \\
\hline
\end{tabular}




\begin{tabular}{|l|l|l|}
\hline Assembly(?y) & \\
artifactHasPart_direct(?x, ?z) & \\
Part(?z) & \\
part2ArtifactAssociation(?y, ?a) & \\
part2ArtifactAssociation(?z, ?a) & \\
\hline
\end{tabular}

Table 1 Artifact Association rules

The first rule is applied when the description of the assembly is complete (the AssemblyAssociation connects two or more Parts) and the assembly has at least one subassembly that is a part. The antecedent of the rule indicates that one Part is directly part of the Assembly while the other one is indirectly connected to the Assembly.

Rule 2 is applied when the description is detailed but the ArtifactAssociation is between Parts that are not directly subassemblies of the Assembly. This means that the Assembly will be composed by other subassemblies that will have Parts that are connected together. We ignore the levels here, because in the antecedent we explore the indirect property to search these parts in the subassemblies.

Rule 3 is applied when the description is not detailed so the Assembly is composed by two or more subassemblies connected together. Rule 4 is similar to the third but is useful in the situations when we want to describe an assembly made by a part and a subassembly.

The highlight of this ontology is that, given the components of the Assembly, we are able to connect the AssemblyAssociation to the Assembly. The drawback now is that we are unable to do the opposite, i.e., to reconstruct the structure of the Assembly having the associations by which it is composed. One solution here is to first input in the rule the property between Assembly and ArtifactAssociations and second the subassemblies that are connected through these associations. Further we do not know at which level of the assembly structure are the subassemblies. Let us take the example of an Assembly A composed by subassemblies $\mathrm{B}$ and $\mathrm{C}$ that are made by Part 1, 2, 3 and 4 respectively; the ArtifactAssociation exists between Part 2 and Part 3. In this case a rule like "if a part is part of an assembly and another part is partof another assembly, then connect these assemblies together to form the superassembly" will hold good, but the problem occurs when the superassembly is composed by three levels. If Assembly A is composed by subassemblies $\mathrm{B}$ and $\mathrm{C}$ that are made respectively by subassemblies D, E, F and G each having two Parts (from 1 to 8) the rule would infer that the Assembly A is defined by Assemblies D and F, without considering the upper level. We then need a rule to recognize the highest level in the hierarchy and then connect the Assemblies in this level with the superassembly through artifactPartof_direct. With the inability to build complex constructs (like OR, NOT or XOR) with SWRL rules, these kind of recognitions are currently impossible. The alternate step is to automatically create the association at the features level.

Table 2 presents the AssemblyFeatureAssociation rules. The first rule is applied when we have a complete description of an artifact, i.e., when the ArtifactAssociation describes the relationship between two Parts. If these Parts have two Features that are 
connected with an AssemblyFeatureAssociation, then this association will be linked to the ArtifactAssociation between the Parts.

\begin{tabular}{|c|c|c|}
\hline Rule & Antecedent & Consequent \\
\hline 1 & $\begin{array}{l}\text { artifactHasFeature(?x,?f) } \\
\text { artifactHasFeature(?y, ?g) } \\
\text { AFA2Feature(?w, ?f) } \\
\text { AFA2Feature(?w, ?g) } \\
\text { artifactAssociation2Part(?z, ?y) } \\
\text { artifactAssociation2Part(?z, ?x) } \\
\text { differentFrom(?x, ?y) } \\
\text { differentFrom(?f, ?g) }\end{array}$ & $\begin{array}{l}\text { AssemblyFeatureAssociation2 } \\
\text { ArtifactAssociation(?w, ?z) }\end{array}$ \\
\hline 2 & $\begin{array}{l}\text { artifactHasFeature(?x, ?f) } \\
\text { artifactHasFeature(?y, ?g) } \\
\text { AFA2Feature(?w, ?f) } \\
\text { AFA2Feature(?w, ?g) } \\
\text { artifactAssociation2Part(?z, ?e) } \\
\text { artifactAssociation2Part(?z, ?d) } \\
\text { artifactHasPart(?d, ?x) } \\
\text { Part(?x) } \\
\text { Assembly(?d) } \\
\text { artifactHasPart(?e, ?y) } \\
\text { Part(?y) } \\
\text { Assembly(?e) } \\
\text { differentFrom(?e, ?d) } \\
\text { differentFrom(?x, ?y) } \\
\text { differentFrom(?f, ?g) }\end{array}$ & $\begin{array}{l}\text { AssemblyFeatureAssociation2 } \\
\text { ArtifactAssociation(?w,?z) }\end{array}$ \\
\hline 3 & $\begin{array}{l}\text { Feature(?f) } \\
\text { Feature(?g) } \\
\text { artifactHasFeature(?x, ?f) } \\
\text { artifactHasFeature(?y, ?g) } \\
\text { ArtifactAssociation(?z) } \\
\text { AssemblyFeatureAssociation(?w) } \\
\text { AFA2Feature(?w, ?f) } \\
\text { AFA2Feature(?w, ?g) } \\
\text { AssemblyFeatureAssociation2 } \\
\text { ArtifactAssociation(?w, ?z) } \\
\text { differentFrom(?x, ?y) } \\
\text { differentFrom(?f, ?g) }\end{array}$ & $\begin{array}{l}\text { artifactAssociation2Part(?z, ?x) } \\
\text { artifactAssociation2Part(?z, ?y) }\end{array}$ \\
\hline
\end{tabular}

Table 2 Assembly Feature Association rules

The second rule is a little bit more complex because it represents the case in which the ArtifactAssociation is between two subassemblies and the Features are relative to the Parts that compose these subassemblies. The logic is the same but in this rule we have to specify the relationship between the features of the Parts of the subassemblies.

The third rule is very similar to the first but the antecedent and the consequent have switched one part. In this case it's possible to say that if two Artifacts have two Features connected together through the AssemblyFeatureAssociation which by itself is linked to an ArtifactAssociation, this last association will link together the Artifacts owning the Features. 
Table 3 presents the AssemblyFeatureAssociationRepresentation rules. The last three rules are consequences of the first. Once a connection is created between two Features with an element of the class AssemblyFeatureAssociation, and association connected with its representation, the first rule will link the representation to the Features. This will be the input for the other rules that will associate the Features with the specification of the representation (KinematicPair, KinematicPath, ParametricAssemblyConstraints).

\begin{tabular}{|l|l|l|}
\hline Rule & \multicolumn{1}{|c|}{ Antecedent } & \multicolumn{1}{|c|}{ Consequent } \\
\hline 1 & $\begin{array}{l}\text { Feature(?f) } \\
\text { AssemblyFeatureAssociationRepresentation(?z) } \\
\text { AssemblyFeatureAssociation(?w) } \\
\text { AFA2Feature(?w, ?f) } \\
\text { AFA_2_AFAR(?w, ?z) }\end{array}$ & AFAR_2_Feature(?z, ?f) \\
\hline \multirow{2}{*}{2} & $\begin{array}{l}\text { Feature(?f) } \\
\text { AssemblyFeatureAssociationRepresentation(?z) } \\
\text { AFAR_2_Feature(?z, ?f) } \\
\text { AFAR_2_KinematicPair(?z, ?w) }\end{array}$ & KinematicPair_2_Feature(?w, ?f) \\
\hline 3 & $\begin{array}{l}\text { Feature(?f) } \\
\text { AssemblyFeatureAssociationRepresentation(?z) } \\
\text { AFAR_2_Feature(?z, ?f) } \\
\text { AFAR_2_KinematicPath(?z, ?w) }\end{array}$ & KinematicPath_2_Feature(?w, ?f) \\
\hline 4 & $\begin{array}{l}\text { Feature(?f) } \\
\text { AssemblyFeatureAssociationRepresentation(?z) } \\
\text { AFAR_2_Feature(?z, ?f) } \\
\text { AFAR_2_ParamAssConstr(?z, ?w) }\end{array}$ & $\begin{array}{l}\text { ParamAssConstr_2_Feature(?w, } \\
\text { ?f) }\end{array}$ \\
\hline
\end{tabular}

Table 3 Assembly Feature Association Representation rules

Table 4 presents the KinematicPath rules. These rules are useful when we want to represent a composite path. In the first two rules a PathElement, connected with a Feature, has either another preceding or succeeding element; as a consequence the two KinematicPaths will be connected to the same Feature. The last two rules are similar to the previous ones but they analyze the structure on a higher level. They connect all the elements in the path to the same AssemblyFeatureAssociationRepresentation.

\begin{tabular}{|l|l|l|}
\hline Rule & \multicolumn{1}{|c|}{ Antecedent } & \multicolumn{1}{c|}{ Consequent } \\
\hline 1 & $\begin{array}{l}\text { PathHasConnection(?x, ?y) } \\
\text { KinematicPath_2_Feature(?x, ?a) } \\
\text { NextPathElement(?y, ?z) }\end{array}$ & KinematicPath_2_Feature(?z, ?a) \\
\hline 2 & $\begin{array}{l}\text { PathHasConnection(?x, ?y) } \\
\text { KinematicPath_2_Feature(?x, ?a) } \\
\text { PreviousPathElement(?y, ?z) }\end{array}$ & KinematicPath_2_Feature(?z, ?a) \\
\hline 3 & $\begin{array}{l}\text { PathHasConnection(?x, ?y) } \\
\text { KinematicPath_2_AFAR(?x, ?a) } \\
\text { NextPathElement(?y, ?z) }\end{array}$ & KinematicPath_2_AFAR(?z, ?a) \\
\hline 4 & $\begin{array}{l}\text { PathHasConnection(?x, ?y) } \\
\text { KinematicPath_2_AFAR(?x, ?a) } \\
\text { PreviousPathElement(?y, ?z) }\end{array}$ & KinematicPath_2_AFAR(?z, ?a) \\
\hline
\end{tabular}

Table 4 Kinematic Path rules 
Table 5 presents the main association rule. This rule infers that if two EntityAsssociations $\mathrm{x}$ and $\mathrm{w}$ connect the same CoreEntities $\mathrm{y}$ and $\mathrm{x}$, then the EntityAssociations are the same. This is necessary because we want to translate a binary property that unequivocally connects two entities.

\begin{tabular}{|l|l|}
\hline \multicolumn{1}{|c|}{ Antecedent } & Consequent \\
\hline entityAssociation2CoreEntity(?x, ?y) & \\
entityAssociation2CoreEntity(?x, ?z) & sameAs(?x, ?w) \\
differentFrom(?y, ?z) & \\
entityAssociation2CoreEntity(?w, ?y) & \\
entityAssociation2CoreEntity(?w, ?z) & \\
\hline
\end{tabular}

Table 5 SameAs rule

We need just one rule because the property classes in the ontology are all children of the EntityAssociation class, so they will adhere to the rule.

There are two partof rules as shown in Table 6. Both are needed to link the Assembly with all its Parts through the indirect property artifactHasPart. The advantage of these rules is that they do not run one after another but with a special algorithm that decomposes the structure of the Assembly. If we have several levels of the Assembly, the algorithm will apply the rules starting from the first subassembly composed by parts.

\begin{tabular}{|l|l|c|}
\hline Rule & \multicolumn{1}{|c|}{ Consequent } & \multicolumn{1}{|c|}{ Antecedent } \\
\hline 1 & $\begin{array}{l}\text { artifactHasPart_direct(?x, ?y) } \\
\text { Part(?y) }\end{array}$ & artifactHasPart(?x, ?y) \\
\hline 2 & $\begin{array}{l}\text { artifactHasPart_direct(?x, ?y) } \\
\text { Assembly(?y) } \\
\text { artifactHasPart(?y, ?z) } \\
\text { Part(?z) }\end{array}$ & artifactHasPart(?x, ?z) \\
\hline
\end{tabular}

Table 6 PartOf rules

The last group of rules in Table 7 are needed to specify if the user explicitly wants to build something without a meaning. The first two rules provide a case in which an Artifact is a subassembly of itself, direct or not. The rules in Table 8 concerns the PairFrames. For a case in which we have a KinematicPair between two Features, the frames of this pair have to be associated with the same Features. Here, we can not use dynamic ranges or restrictions for the properties and hence we cannot constrain the user to specifically choose only between the Features that are connected with a KinematicPair. For this reason the rule will consider a PairFrame as meaningless when the user does something logically incorrect or when he uses a Feature to describe the frame even if the Feature is not included in the KinematicPair. 


\begin{tabular}{|l|l|l|}
\hline Rule & \multicolumn{1}{|c|}{ Antecedent } & \multicolumn{1}{c|}{ Consequent } \\
\hline 1 & artifactHasPart_direct(?x, ?x) & meaning_less_artifact(?x) \\
\hline 2 & artifactHasPart(?x, ?x) & meaning_less_artifact(?x) \\
\hline
\end{tabular}

Table 7 Rules for not Allowed Artifacts

\begin{tabular}{|l|c|}
\hline \multicolumn{1}{|c|}{ Antecedent } & \multicolumn{1}{|c|}{ Consequent } \\
\hline KinematicPair_2_Feature(?k, ?f) & \\
KinematicPair_2_Feature(?k, ?g) & \\
differentFrom(?f, ?g) & PairFrame_meaning_less(?x) \\
pair_frame(?k, ?x) & \\
PairFrameAttribute2Feature(?x, ?h) & \\
differentFrom(?f, ?h) & \\
differentFrom(?g, ?h) & \\
\hline
\end{tabular}

Table 8 Rule for not allowed Pair Frame

After defining the model, the restrictions and the SWRL rules, the next step is to verify if it is well composed and if it can represent every condition/assembly. For this reason we choose a use case to underline advantages and disadvantages of the OWL version of the Open Assembly Model.

\section{Case study: Planetary Gear System}

This section illustrates the implementation of an industrial example used to test the OAM ontology. The assembly model of a planetary gear system is modeled using a Computer Aided Design (CAD) system. Section 6.1 is dedicated to explain the reasons that have led to the selection of this particular assembly and related description. Section 6.2 presents the implementation and instantiation of the model, together with the reasoning capabilities performed by RACER and Jess.

\subsection{Use Case Description}

The Planetary Gear System is an electromechanical component normally used to change the rotation speed or the torque of a shaft. In this example our aim is to represent a scenario of an assembly representation to outline assembly complexity but at the same time not to complicate the example itself. Moreover, the same example had been previously used during the instantiation of the OAM-UML model.

The System consists of 4 subassemblies with almost 30 different parts. As with any electromechanical component, tolerances are specifically defined for all parts. In the chosen planetary gear system, the connection and pairs between different artifacts are of different types. 


\subsubsection{Components in Planetary Gear System}

The solid model of the planetary gear system is shown in Figure 19.
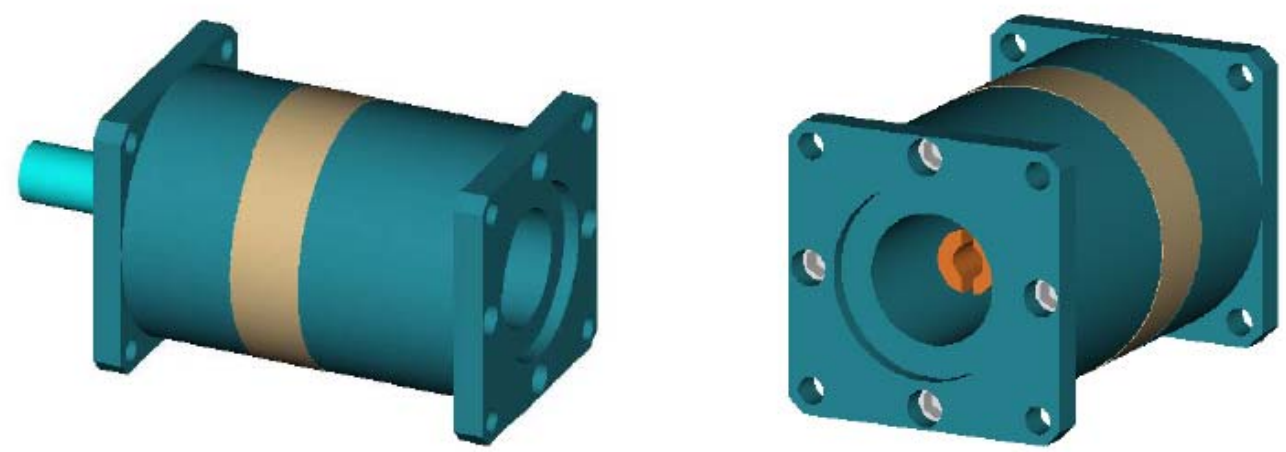

Figure 19 Planetary Gear System

The planetary gear system consists of many components. Figure 20 shows the exploded view of the above solid model. The list of all the components of the planetary gear system is given in Table 9 .

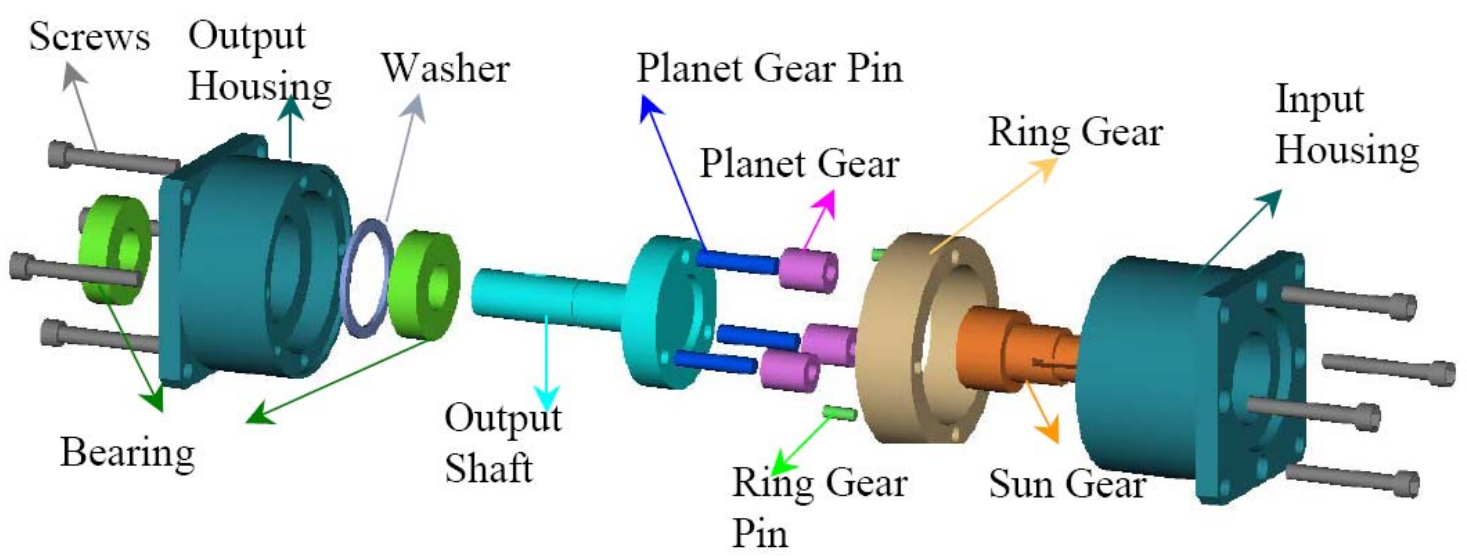

Figure 20 Exploded view of the Planetary Gear System

\subsubsection{Assembly Hierarchy}

We first need to define an assembly hierarchy for the planetary gear system. The planetary gear system is composed of two parts and three sub-assemblies as shown in Figure 20. The parts include the input-housing and the sungear. The three subassemblies include: (1) the output end assembly comprising two bearings, a washer, and the output housing; (2) the ring gear assembly comprising a ring gear and two ring-gear pins; and (3) the planet gear holder assembly comprising three planet gears and a planet carrier assembly, which further decomposes into the output shaft and three planet-gear pins. 


\begin{tabular}{|c|c|c|c|c|}
\hline ID & Name & Qty & Functional Description & $\begin{array}{l}\text { Graphical } \\
\text { Representation }\end{array}$ \\
\hline 1 & $\begin{array}{l}\text { Output } \\
\text { Housing }\end{array}$ & 1 & $\begin{array}{l}\text { Covers output shaft and protects the gears } \\
\text { and shafts }\end{array}$ & \\
\hline 2 & Bearing & 2 & $\begin{array}{l}\text { Supports the output housing and serves as an } \\
\text { interface between the output shaft and the } \\
\text { housing }\end{array}$ & \\
\hline 3 & Washer & 1 & $\begin{array}{l}\text { Separate the two bearings inside the output } \\
\text { housing }\end{array}$ & \\
\hline 4 & Output shaft & 1 & $\begin{array}{l}\text { Transmits power to the driven device. Also, } \\
\text { connects to planetary gears. }\end{array}$ & \\
\hline 5 & $\begin{array}{l}\text { Planet gear } \\
\text { pin }\end{array}$ & 3 & $\begin{array}{l}\text { Holds a planetary gear and attaches it to the } \\
\text { output shaft }\end{array}$ & \\
\hline 6 & Planet gear & 3 & $\begin{array}{l}\text { Delivers power from the sungear to the } \\
\text { output shaft }\end{array}$ & \\
\hline 7 & Ring gear & 1 & $\begin{array}{l}\text { Controls the speed reduction ratio. The } \\
\text { planetary gears rotate around it. }\end{array}$ & \\
\hline 8 & $\begin{array}{l}\text { Ring gear } \\
\text { pin }\end{array}$ & 2 & Attaches the ring gear to the output housing & \\
\hline 9 & Sungear & 1 & $\begin{array}{l}\text { Transmits the power from input shaft to } \\
\text { planetary gears. Input shaft and sungear } \\
\text { considered as one part. }\end{array}$ & \\
\hline 10 & $\begin{array}{l}\text { Input } \\
\text { housing }\end{array}$ & 1 & $\begin{array}{l}\text { Covers the input shaft and provides } \\
\text { protection from } \\
\text { contamination. }\end{array}$ & \\
\hline 11 & Screw & 8 & $\begin{array}{l}\text { Fastens the input housing, the ring gear, and } \\
\text { the output housing into one assembly. }\end{array}$ & \\
\hline
\end{tabular}

Table 9 Components of the Planetary Gear System 


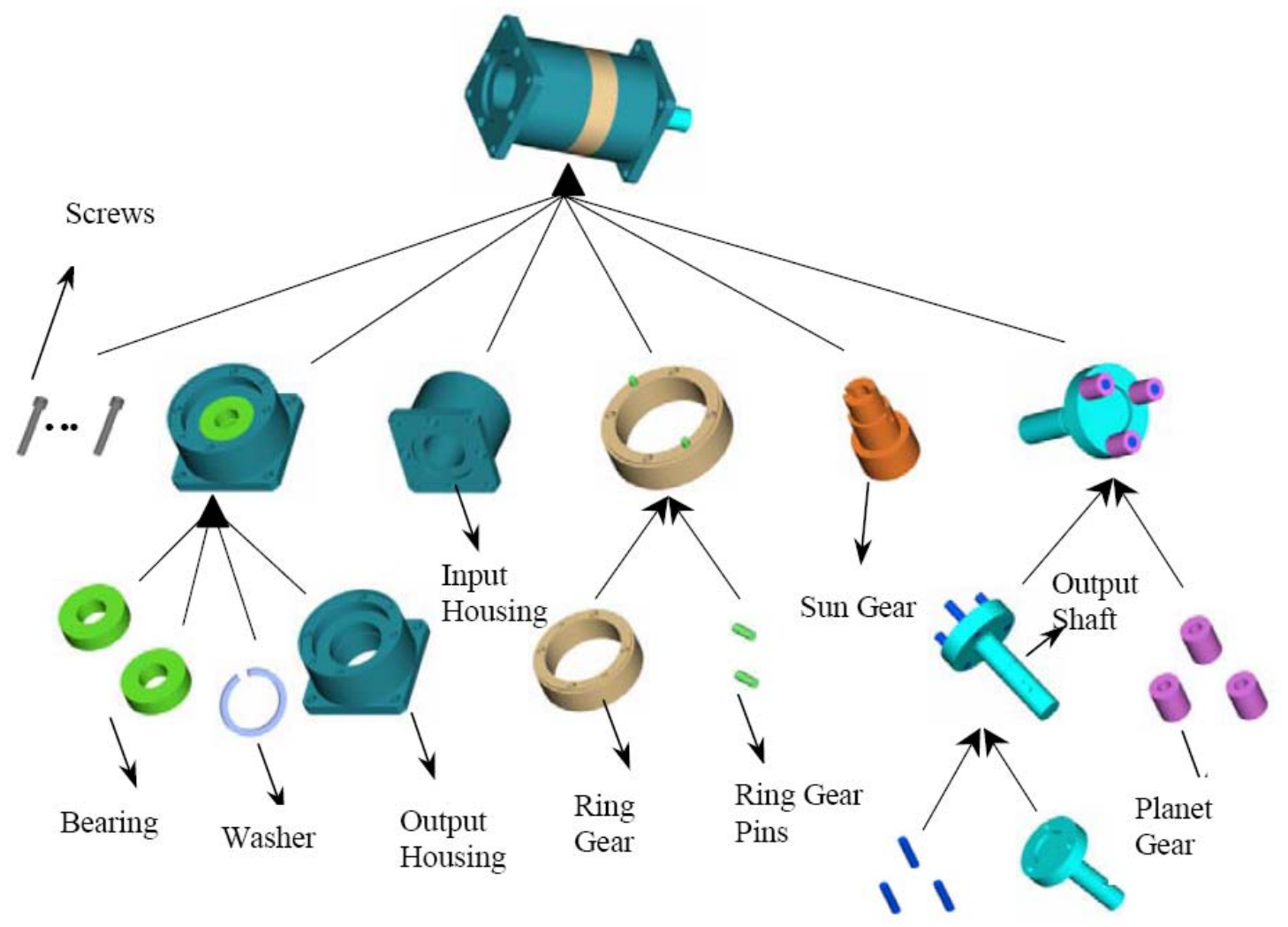

Figure 21 Planetary Gear structure

The hierarchical relationships between the components of the planetary gear system can be represented as an instance diagram as shown in Figure 22. The names take the form of "instance name:class name". The root node is the entire assembly, the interior nodes are sub- assemblies, and the leaf nodes are component parts. 


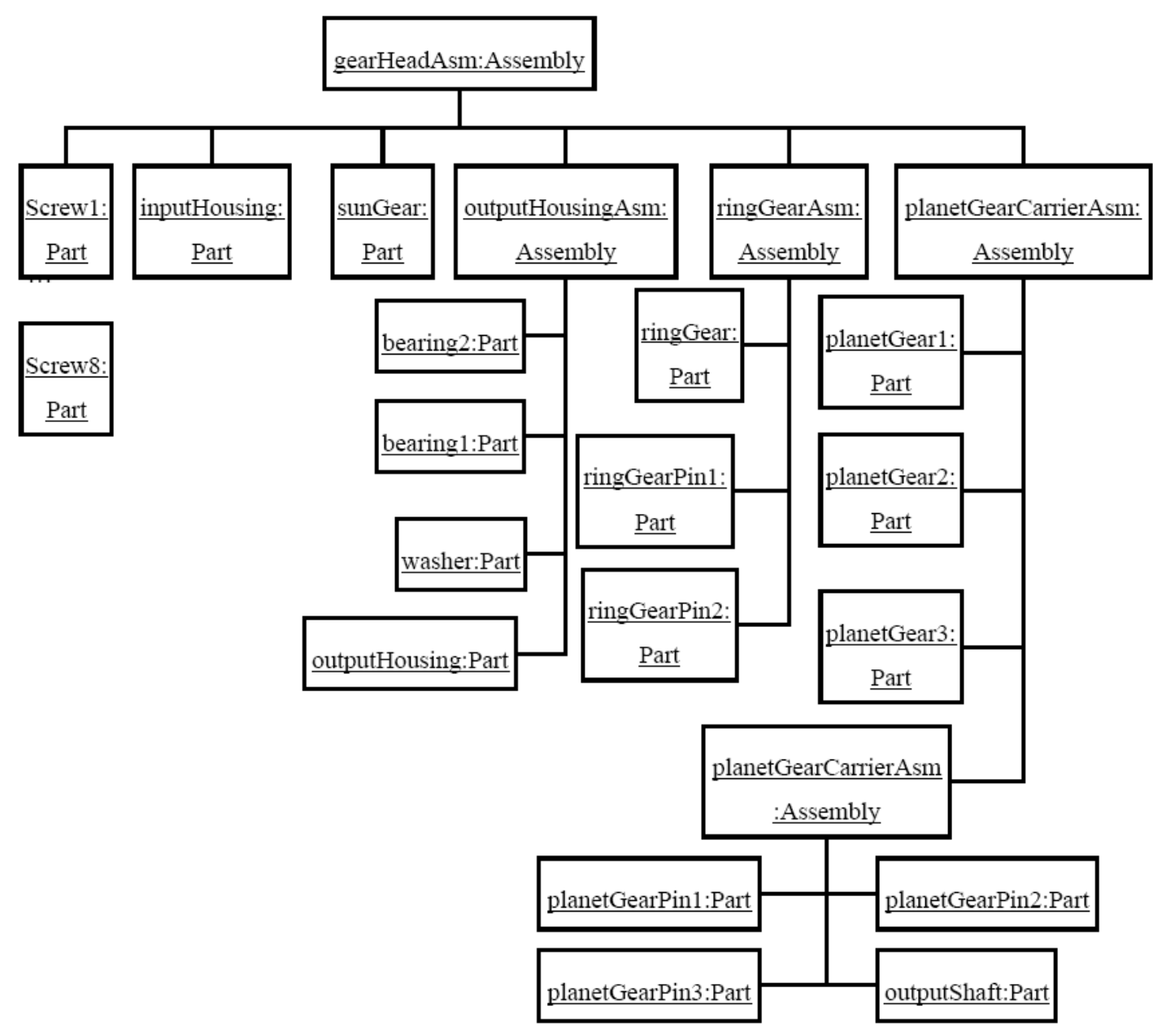

Figure 22 Planetary Gear hierarchy

The connections between parts are presented in the Figure 23. The naming conventions are related to the types of possible connections (fc: fixed connection, mc: movable connection, po: position orientation). These connections between parts are represented in the model through instances of the class ArtifactAssociation. 


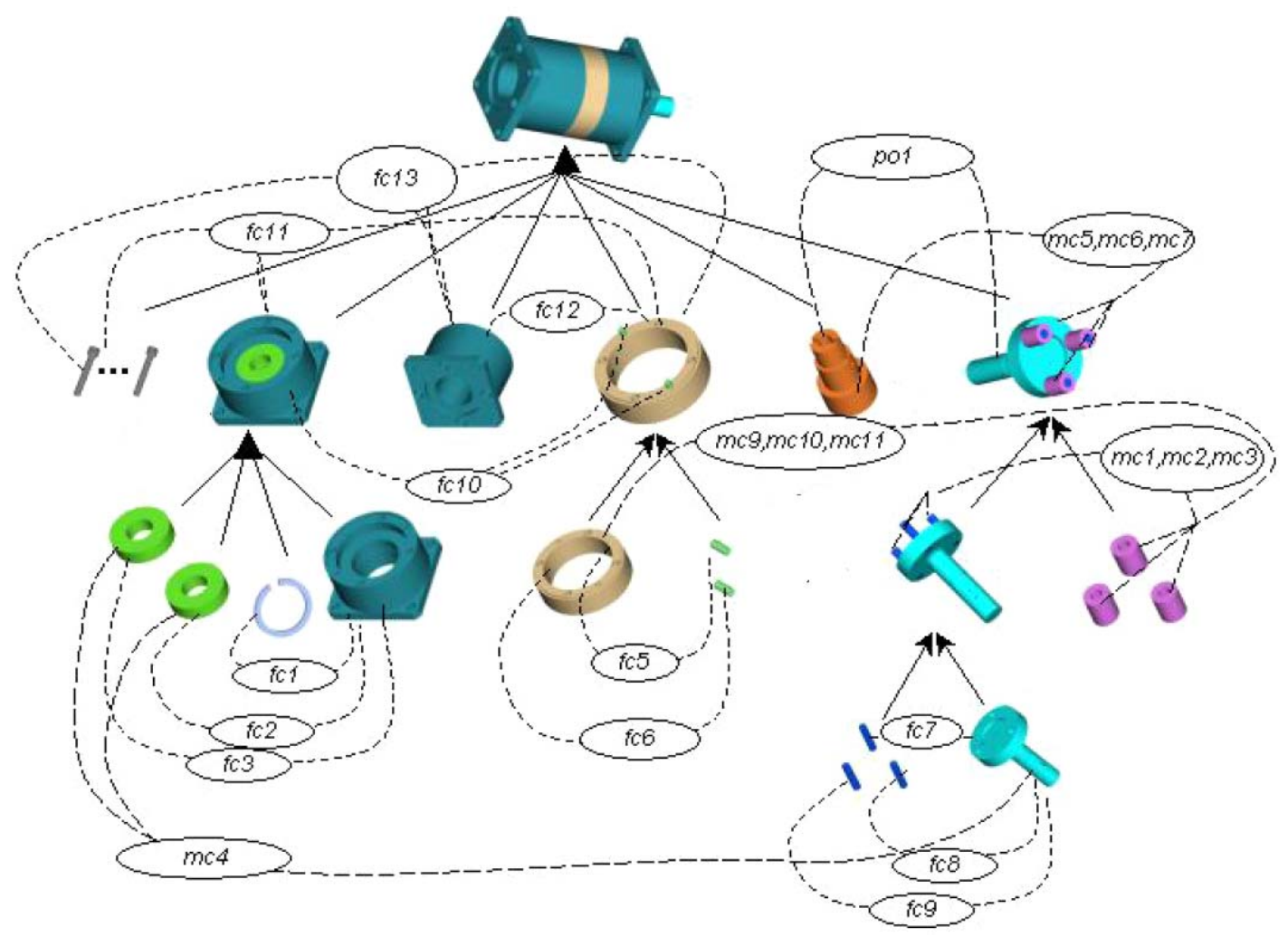

Figure 23 Connections between parts

\subsubsection{Output Housing Assembly}

Figure 24 shows the subassembly and the output housing (the output end of the planetary gear system). It consists of four parts: bearing 1, bearing 2, washer, and output housing. The washer goes to the inner groove of the output housing. Both bearings (ball bearings) go into the output housing on either side of the washer with a tight fit. Bearing 1 stays outside, and Bearing 2 stays inside of the planetary gear system.

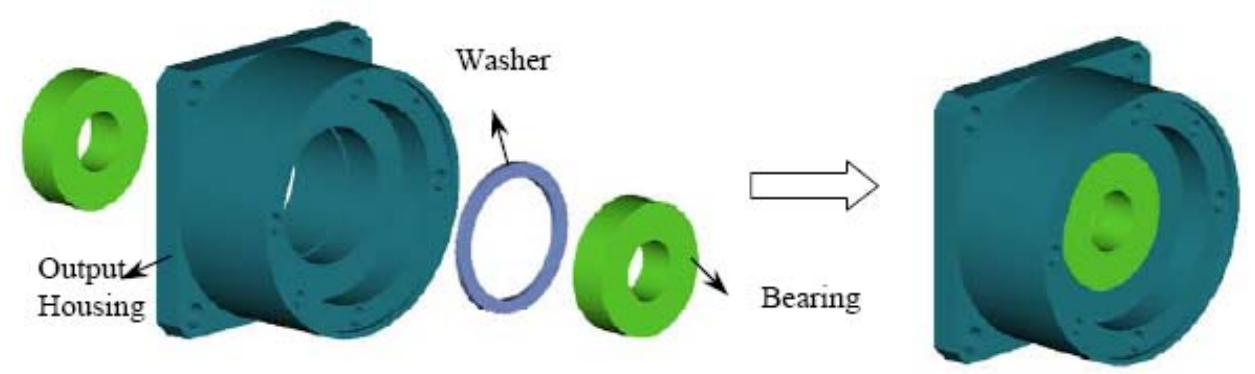

Figure 24 Output Housing Assembly 


\subsubsection{Ring Gear Assembly}

The second subassembly is the ring gear assembly, shown in Figure 25. It consists of three parts: ring gear, and ring-gear pins 1 and 2. The two ring-gear pins go into the pinholes of the ring gear with a tight fit.
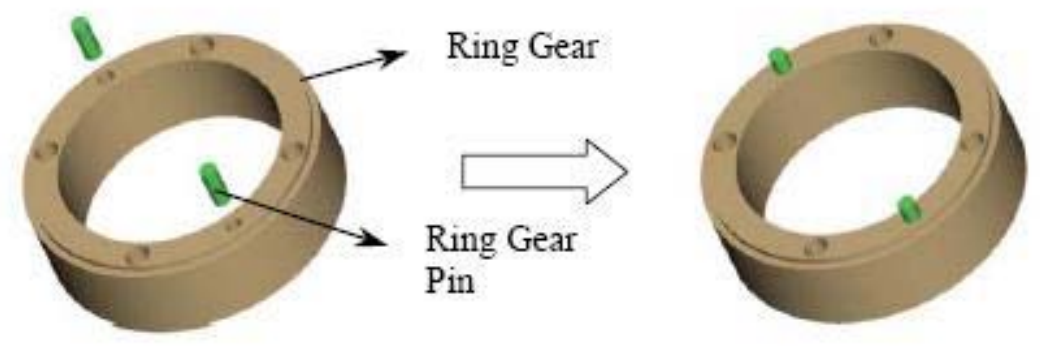

Figure 25 Ring Gear Assembly

\subsubsection{Planet Gear-carrier Assembly}

The planet gear-carrier assembly shown in Figure 26 is comprised of four parts: three planet gears and one planet carrier assembly. The three planet gears are assembled by a loose fit with the planet-gear pins of the planet carrier assembly.
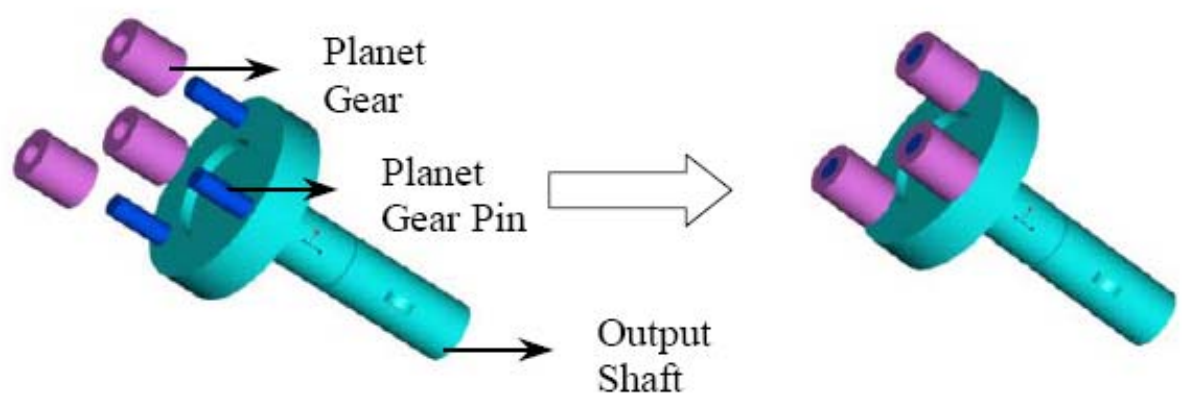

Figure 26 Planet Gear-carrier Assembly

\subsubsection{Planet Carrier Assembly and Sungear}

The planet carrier assembly in Figure 27 is comprised of four parts: three planet-gear pins and an output shaft. The three planet-gear pins are assembled with output shaft by a tight fit. The sungear is assembled with the three planet gears of the planet gear-carrier assembly by gear meshing. 


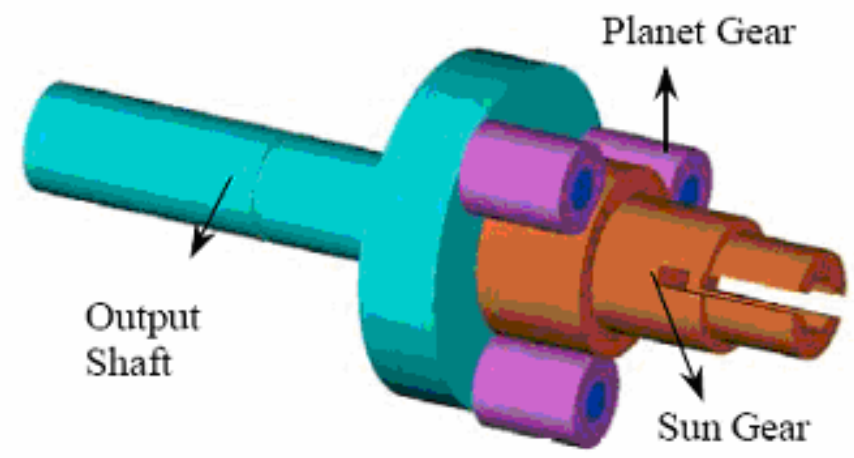

Figure 27 Planet Carrier Assembly and Sungear

\subsubsection{Output Housing Assembly}

Consider the output housing assembly and planet gear-carrier assembly shown in Figure 28.

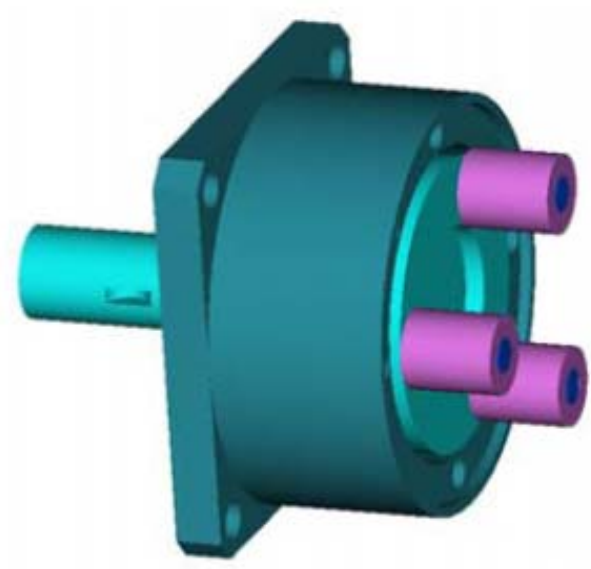

Figure 28 Output Housing Assembly

The output shaft of the planet gear-assembly is inserted into the bearings of the output housing assembly.

\subsection{Use Case Implementation}

In this section, the use case implementation is presented. For explanation of the Planetary Gear System example, the structure of the ontology will be followed and every class will be presented twice with its instances and properties. Accordingly, this section is divided into two main parts: Input Instances and Output Instances. Note that the whole model is composed by 145 classes, 200 properties, 70 restrictions on properties used for the class definitions and 25 different SWRL rules. The classes that are omitted in this explanation of the OAM ontology are either simply used to store information or are simple 
specifications of the presented classes or properties. The total number of instances needed for the use case description is approximately 250.

\subsubsection{Asserted Instances and Properties}

In this section, each class is presented with the instances and the input properties needed for the basic population of the model. The elements inserted in the models are necessary for the subsequent reasoning on the ontology. In most cases, the properties in the model have an inverse: the user has to only instantiate the property on one direction because the other is automatically constituted by the editor. In the following tables all the properties for every class are shown.

\subsubsection{Asserted Artifact Instances and Properties}

The class Artifact has four subclasses: Assembly, Meaningless_Artifact, Connecter and Part. The reasoning capabilities of the model allow us to create all the instances of Assembly directly as Artifacts and then infer them as instances of Assembly. This is possible defining an Assembly as an Artifact composed by at least two subassemblies (through restriction on the property artifactHasPart_direct.

The input instances of the class Artifact are presented in Table 10. In the first column of the table are the instances of artifact. In the other columns, are the instances of the related classes linked through the property that names these columns. From the table, notice that the Output_Housing_Assembly is incorrectly defined i.e., composed by itself (*). This error is purposely introduced for testing the reasoning capabilities of the ontology in sections 5.2.1.3 (Meaning_Less_Artifact Input) and 5.2.2.3 (Meaning_Less_Artifact Output).

\begin{tabular}{|c|c|c|}
\hline \multirow[b]{2}{*}{ Artifact Instances } & \multicolumn{2}{|c|}{ Asserted Properties } \\
\hline & $\begin{array}{l}\text { artifactHasPart_direct } \\
\text { (D:Artifact R:Artifact) }\end{array}$ & $\begin{array}{l}\text { cpm2:partOfArtifact_direct } \\
\text { (D:Artifact R:Artifact) }\end{array}$ \\
\hline Output_Housing_Assembly & $\begin{array}{l}\text { Bearing_1 } \\
\text { Bearing_2 } \\
\text { Output_Housing } \\
\text { Washer } \\
\text { Output_Housing_Assembly* }\end{array}$ & $\begin{array}{l}\text { Planetary_Gear } \\
\text { System_Assembly }\end{array}$ \\
\hline Planet_Carrier_Assembly & $\begin{array}{l}\text { Output_Shaft } \\
\text { Planet_Gear_Pin_1 } \\
\text { Planet_Gear_Pin_2 } \\
\text { Planet_Gear_Pin_3 }\end{array}$ & $\begin{array}{l}\text { Planetary_Gear } \\
\text { Carrier_Assembly }\end{array}$ \\
\hline $\begin{array}{l}\text { Planet_Gear } \\
\text { Carrier_Assembly }\end{array}$ & $\begin{array}{l}\text { Planet_Gear_1 } \\
\text { Planet_Gear_2 } \\
\text { Planet_Gear_3 } \\
\text { Planet_Carrier_Assembly }\end{array}$ & $\begin{array}{l}\text { Planetary_Gear } \\
\text { System_Assembly }\end{array}$ \\
\hline Ring_Gear_Assembly & $\begin{array}{l}\text { Ring_Gear } \\
\text { Ring_Gear_Pin_1 } \\
\text { Ring_Gear_Pin_2 }\end{array}$ & $\begin{array}{l}\text { Planetary_Gear } \\
\text { System_Assembly }\end{array}$ \\
\hline $\begin{array}{l}\text { Planetary_Gear } \\
\text { System_Assembly }\end{array}$ & $\begin{array}{l}\text { Output_Housing_Assembly } \\
\text { Planet_Carrier_Assembly } \\
\text { Planet_Gear_Carrier_Assembly }\end{array}$ & -- \\
\hline
\end{tabular}




\begin{tabular}{|l|l|l|}
\hline & Ring_Gear_Assembly & \\
& Sungear & \\
& Input_Housing & \\
& Screw_1 & \\
Screw_2 & & \\
& Screw_3 & \\
Screw_4 & \\
Screw_5 & \\
& Screw_6 & \\
& Screw_7 & \\
Screw_8 & \\
\hline
\end{tabular}

Table 10 Artifact: asserted instances and properties

\subsubsection{Asserted Assembly Instances and Properties}

It is possible to assert all instances of the class Assembly as instances of the class Artifact and let the reasoner (in this case RACER) reclassify the instances. At this point the class Assembly is empty.

\subsubsection{Asserted Meaning_Less_Artifact Instances and Properties}

This class is created for managing the impossibility of blocking the creation of a selfreference in the current version of Protégé-OWL. In the current model it is possible to define an instance of Assembly composed by itself. Presently, there is no direct solution and hence the class "Meaning_Less_Artifact" is created. For demonstration purposes the wrong definition of the Output_Housing_Assembly is introduced for testing the capability of the ontology to identify this kind of error (see 5.2.1.1 for details). Thanks to the SWRL rules (see section 4.2 for details) created with an aim to reclassify the wrongly defined instances (see section 5.2.2.3 for details). At the instantiation step this class is empty.

\subsubsection{Asserted Part Instances and Properties}

Although the class Part is a subclass of the class Artifact, it is not possible to assert the instances of the different parts as instances of Artifact and later infer them as instances of Part as with the case of the instances of Assembly. This is due to the limitation with OWL: it is impossible to define a class as a class without a property. An instance of Part is an Artifact that is not composed by any other Parts but for a reasoner an Artifact without a property (in this case artifactHasPart_direct) is not an instance of Part but only an instance of Artifact not yet completely defined. For this reason all the parts are created directly in the class Part.

The asserted instances and properties for the class Part are shown in Table 11. 


\begin{tabular}{|c|c|c|c|}
\hline \multirow[b]{2}{*}{ Part Instances } & \multicolumn{3}{|c|}{ Asserted Properties } \\
\hline & $\begin{array}{l}\text { partOfArtifact_direct } \\
\text { (D:Artifact R:Artifact) }\end{array}$ & $\begin{array}{l}\text { artifactHasFeature } \\
\text { (D:Artifact } \\
\text { R:Feature) }\end{array}$ & $\begin{array}{l}\text { part2ArtifactAssociation } \\
\text { (D:Part } \\
\text { R:ArtifactAssociation) }\end{array}$ \\
\hline Bearing_1 & Output_Housing_Assembly & $\begin{array}{l}\text { Inner_Race_1 } \\
\text { Outer_Race_1 }\end{array}$ & $\begin{array}{l}f c \_2 \\
m c \_4\end{array}$ \\
\hline Bearing_2 & Output_Housing_Assembly & $\begin{array}{l}\text { Inner_Race_2 } \\
\text { Outer_Race_2 }\end{array}$ & $\begin{array}{l}f c \_3 \\
m c \_4\end{array}$ \\
\hline Input_Housing & Planetary_Gear_System_Assembly & $\begin{array}{l}\text { Stepped_Side } \\
\text { Thru_Hole_5 } \\
\text { Thru_Hole_6 } \\
\text { Thru_Hole_7 } \\
\text { Thru_Hole_8 }\end{array}$ & $\begin{array}{l}f c \_12 \\
f c \_13\end{array}$ \\
\hline Output_Housing & Output_Housing_Assembly & $\begin{array}{l}\text { Bearing_Seat_1 } \\
\text { Bearing_Seat_2 } \\
\text { Groove } \\
\text { pin_Hole_9 } \\
\text { pin_Hole_10 } \\
\text { Thru_Hole_1 } \\
\text { Thru_Hole_2 } \\
\text { Thru_Hole_3 } \\
\text { Thru_Hole_4 }\end{array}$ & $\begin{array}{l}f c \_1 \\
f c \_2 \\
f c \_3 \\
f c \_4 \\
f c \_10 \\
f c \_11\end{array}$ \\
\hline Output_Shaft & Planet_Carrier_Assembly & $\begin{array}{l}\text { Bearing_Seat_3 } \\
\text { Output_Shaft_Feature } \\
\text { pin_Hole_3 } \\
\text { pin_Hole_4 } \\
\text { pin_Hole_5 }\end{array}$ & $\begin{array}{l}f c \_7 \\
f c \_8 \\
f c \_9 \\
m c \_4 \\
\text { po_1 }\end{array}$ \\
\hline Planet_Gear_1 & Planet_Gear_Carrier_Assembly & $\begin{array}{l}\text { pin_Cylinder_3 } \\
\text { teeth_7 } \\
\text { teeth_8 }\end{array}$ & $\begin{array}{l}m c \_1 \\
m c \_5 \\
m c \_9\end{array}$ \\
\hline Planet_Gear_2 & Planet_Gear_Carrier_Assembly & $\begin{array}{l}\text { pin_Hole_7, teeth_9, } \\
\text { teeth_10 }\end{array}$ & $m c \_2, m c \_6, m c \_10$ \\
\hline Planet_Gear_3 & Planet_Gear_Carrier_Assembly & $\begin{array}{l}\text { pin_Hole_8, teeth_11, } \\
\text { teeth_12 }\end{array}$ & $m c \_3, m c \_7, m c \_11$ \\
\hline Planet_Gear_Pin_1 & Planet_Carrier_Assembly & $\begin{array}{l}\text { pin_Cylinder_3, } \\
\text { pin_Cylinder_6 }\end{array}$ & $m c \_1, f c \_5, f c \_7$ \\
\hline Planet_Gear_Pin_2 & Planet_Carrier_Assembly & $\begin{array}{l}\text { pin_Cylinder_4, } \\
\text { pin_Cylinder_7 }\end{array}$ & $m c \_2, f c \_8$ \\
\hline Planet_Gear_Pin_3 & Planet_Carrier_Assembly & $\begin{array}{l}\text { pin_Cylinder_5, } \\
\text { pin_Cylinder_8 }\end{array}$ & mc_3, fc_9 \\
\hline Ring_Gear & Ring_Gear_Assembly & $\begin{array}{l}\text { pin_Hole_1, } \\
\text { pin_Hole_2 } \\
\text { Ring_Gear_Side } \\
\text { teeth_4, } \quad \text { teeth_5, } \\
\text { teeth_6 } \\
\text { threaded_Hole_1 } \\
\text { hreaded_Hole_2 } \\
\text { threaded_Hole_3 } \\
\text { threaded_Hole_4 }\end{array}$ & $\begin{array}{l}m c \_9, m c \_10 \\
m c \_11, f c \_5 \\
f c \_6, f c \_11 \\
f c \_12, f c \_13\end{array}$ \\
\hline Ring_Gear_Pin_1 & Ring_Gear_Assembly & $\begin{array}{l}\text { pin_Cylinder_1, } \\
\text { pin_Cylinder_5 } \\
\text { pin_Cylinder_9 }\end{array}$ & $\begin{array}{l}f c \_5 \\
f c \_10\end{array}$ \\
\hline Ring_Gear_Pin_2 & Ring_Gear_Assembly & $\begin{array}{l}\text { pin_Cylinder_2, } \\
\text { pin_Cylinder_7 } \\
\text { pin_Cylinder_10 }\end{array}$ & $\begin{array}{l}f c \_6 \\
f c \_10\end{array}$ \\
\hline Screw_1 & Planetary_Gear_System_Assembly & thread_1 & fc_11 \\
\hline Screw_2 & Planetary_Gear_System_Assembly & thread_2 & fc_11 \\
\hline
\end{tabular}




\begin{tabular}{|l|l|l|l|}
\hline Screw_3 & Planetary_Gear_System_Assembly & thread_3 & $f c \_11$ \\
\hline Screw_4 & Planetary_Gear_System_Assembly & thread_4 & $f c \_11$ \\
\hline Screw_5 & Planetary_Gear_System_Assembly & thread_5 & fc_11 \\
\hline Screw_6 & Planetary_Gear_System_Assembly & thread_6 & fc_11 \\
\hline Screw_7 & Planetary_Gear_System_Assembly & thread_7 & fc_11 \\
\hline Screw_8 & Planetary_Gear_System_Assembly & thread_8 & fc_11 \\
\hline Washer & Output_Housing_Assembly & Outer_Rim & fc_1 \\
\hline & & $\begin{array}{l}\text { DatumFeature_Axis1 } \\
\text { Sun_Gear_Feature, } \\
\text { Sungearth_1, } \\
\text { teeth_2, teeth_3, } \\
\text { Input_Shaft }\end{array}$ & $\begin{array}{l}\text { mc_5, mc_6, mc_7 } \\
\text { mo_1 }\end{array}$ \\
\hline
\end{tabular}

Table 11 Part: asserted instances and properties

\subsubsection{Asserted Features Instances and Properties}

The class Feature has the same level of the class Artifact (both of them are children of CoreEntity)and stores the instances that represent the features of the single parts. This class has two direct subclasses Port and OAMFeature. Class OAMFeature further has two subclasses used to represent the reference features as DatumFeatures. Table 12 presents the instances of the class OAMFeatures that participate in the creation of the assemblies through the different types of connections.

\begin{tabular}{|l|l|l|l|}
\hline \multirow{2}{*}{$\begin{array}{l}|c| \\
\text { OAMFeatures } \\
\text { Instances }\end{array}$} & $\begin{array}{l}\text { FeatureOfArtifact } \\
\text { (D:Feature R:Artifact) }\end{array}$ & $\begin{array}{l}\text { feature2AFA } \\
\text { (D:Feature R:AFA) }\end{array}$ & $\begin{array}{l}\text { feature2AFAR } \\
\text { (D:Feature R:AFAR) }\end{array}$ \\
\hline Bearing_Seat_1 & Output_Housing & AFA_fc2 & AFAR_fc2 \\
\hline Bearing_Seat_2 & Output_Housing & AFA_fc3 & AFAR_fc3 \\
\hline Bearing_Seat_3 & Output_Shaft & AFA_mc4 & AFAR_mc4 \\
\hline Groove & Output_Housing & AFA_fc1 & AFAR_fc1 \\
\hline Inner_Race_1 & Bearing_1 & AFA_mc4 & AFAR_mc4 \\
\hline Inner_Race_2 & Bearing_2 & AFA_mc4 & AFAR_mc4 \\
\hline Outer_Race_1 & Bearing_1 & AFA_fc2 & AFAR_fc2 \\
\hline Outer_Race_2 & Bearing_2 & AFA_fc3 & AFAR_fc3 \\
\hline Outer_Rim & Washer & AFA_fc1 & AFAR_fc1 \\
\hline Output_Shaft_Feature & Output_Shaft & AFA_po1 & AFAR_po1 \\
\hline pin_Cylinder_1 & Ring_Gear_Pin_1 & AFA_fc5 & AFAR_fc5 \\
\hline pin_Cylinder_2 & Ring_Gear_Pin_2 & AFA_fc6 & AFAR_fc6 \\
\hline pin_Cylinder_3 & Planet_Gear_1 & AFA_fc7 & AFAR_fc7 \\
\hline pin_Cylinder_4 & Planet_Gear_Pin_2 & AFA_fc8 & AFAR_fc8 \\
\hline pin_Cylinder_5 & Planet_Gear_Pin_3 & AFA_fc9 & AFAR_fc9 \\
\hline & & & \\
\hline
\end{tabular}




\begin{tabular}{|c|c|c|c|}
\hline pin_Cylinder_6 & Ring_Gear_Pin_1 & $A F A \_m c 1$ & AFAR_mc1 \\
\hline pin_Cylinder_7 & Planet_Gear_Pin_2 & $A F A \_m c 2$ & AFAR_mc2 \\
\hline pin_Cylinder_8 & Planet_Gear_Pin_3 & AFA_mc3 & AFAR_mc3 \\
\hline pin_Cylinder_9 & Ring_Gear_Pin_1 & $A F A \_f c 10$ & $A F A R \_f c 10$ \\
\hline pin_Cylinder_10 & Ring_Gear_Pin_2 & $A F A \_f c 10$ & $A F A R \_f c 10$ \\
\hline pin_Hole_1 & Ring_Gear & $A F A \_f c 5$ & $A F A R \_f c 5$ \\
\hline pin_Hole_2 & Ring_Gear & $A F A \_f c 6$ & $A F A R \_f c 6$ \\
\hline pin_Hole_3 & Output_Shaft & $A F A \_f c 7$ & $A F A R \_f c 7$ \\
\hline pin_Hole_4 & Output_Shaft & $A F A \_f c 8$ & $A F A R \_f c 8$ \\
\hline pin_Hole_5 & Output_Shaft & $A F A \_f C 9$ & $A F A R \_f c 9$ \\
\hline Pin_Hole_6 & Ring_Gear_Pin_2 & $A F A \_m c 1$ & $A F A R \_m c 1$ \\
\hline pin_Hole_7 & Planet_Gear_2 & $A F A \_m c 2$ & $A F A R \_m c 2$ \\
\hline pin_Hole_8 & Planet_Gear_3 & $A F A \_m c 3$ & $A F A R \_m c 3$ \\
\hline pin_Hole_9 & Output_Housing & $A F A \_f c 10$ & $A F A R \_f C 10$ \\
\hline pin_Hole_10 & Output_Housing & $A F A \_f c 10$ & $A F A R \_f C 10$ \\
\hline Ring_Gear_Side & Ring_Gear & $A F A \_f c 12$ & $A F A R \_f C 12$ \\
\hline Stepped_Side & Input_Housing & $A F A \_f c 12$ & $A F A R \_f C 12$ \\
\hline Sun_Gear_Feature & Sungear & AFA_po1 & $A F A R \_p o 1$ \\
\hline teeth_1 & Sungear & $A F A \_m c 5$ & $A F A R \_m c 5$ \\
\hline teeth_2 & Sungear & $A F A \_m c 6$ & $A F A R \_m c 6$ \\
\hline teeth_3 & Sungear & $A F A \_m c 7$ & $A F A R \_m c 7$ \\
\hline teeth_4 & Ring_Gear & $A F A \_m c 9$ & AFAR_mc9 \\
\hline teeth_5 & Ring_Gear & AFA_mc10 & AFAR_mc10 \\
\hline teeth_6 & Ring_Gear & AFA_mc11 & AFAR_mc11 \\
\hline teeth_7 & Planet_Gear_1 & $A F A \_m c 5$ & AFAR_mc5 \\
\hline teeth_8 & Planet_Gear_1 & $A F A \_m c 9$ & AFAR_mc9 \\
\hline teeth_9 & Planet_Gear_2 & $A F A \_m c 6$ & AFAR_mc6 \\
\hline teeth_10 & Planet_Gear_2 & AFA_mc10 & AFAR_mc10 \\
\hline teeth_11 & Planet_Gear_3 & $A F A \_m c 7$ & $A F A R \_m c 7$ \\
\hline teeth_12 & Planet_Gear_3 & AFA_mc11 & AFAR_mc11 \\
\hline thread_1 & Screw_1 & $A F A \_f c 11$ & $A F A R \_f C 11$ \\
\hline thread_2 & Screw_2 & $A F A \_f c 11$ & $A F A R \_f c 11$ \\
\hline thread_3 & Screw_3 & $A F A \_f c 11$ & $A F A R \_f c 11$ \\
\hline thread_4 & Screw_4 & $A F A \_f c 11$ & $A F A R \_f c 11$ \\
\hline thread_5 & Screw_5 & $A F A \_f c 13$ & AFAR_fc13 \\
\hline thread_6 & Screw_6 & $A F A \_f c 13$ & AFAR_fc13 \\
\hline thread_7 & Screw_7 & $A F A \_f c 13$ & AFAR_fc13 \\
\hline thread_8 & Screw_8 & $A F A \_f c 13$ & AFAR_fc13 \\
\hline \multirow{2}{*}{ threaded_Hole_1 } & \multirow{2}{*}{ Ring_Gear } & $A F A \_f c 11$ & $A F A R \_f c 11$ \\
\hline & & $A F A \_f c 13$ & $A F A R \_f C 13$ \\
\hline
\end{tabular}




\begin{tabular}{|c|c|c|c|}
\hline \multirow{2}{*}{ threaded_Hole_2 } & \multirow{2}{*}{ Ring_Gear } & AFA_fC11 & $A F A R \_f C 11$ \\
\hline & & $A F A \_f c 13$ & $A F A R \_f C 13$ \\
\hline \multirow{2}{*}{ threaded_Hole_3 } & \multirow{2}{*}{ Ring_Gear } & AFA_fC11 & $A F A R \_f C 11$ \\
\hline & & $A F A \_f c 13$ & $A F A R \_f C 13$ \\
\hline \multirow{2}{*}{ threaded_Hole_4 } & \multirow{2}{*}{ Ring_Gear } & $A F A \_f c 11$ & $A F A R \_f C 11$ \\
\hline & & $A F A \_f c 13$ & $A F A R \_f C 13$ \\
\hline Thru_Hole_1 & Output_Housing & $A F A \_f C 11$ & $A F A R \_f C 11$ \\
\hline Thru_Hole_2 & Output_Housing & $A F A \_f c 11$ & $A F A R \_f C 11$ \\
\hline Thru_Hole_3 & Output_Housing & $A F A \_f c 11$ & $A F A R \_f C 11$ \\
\hline Thru_Hole_4 & Output_Housing & $A F A \_f c 11$ & $A F A R \_f C 11$ \\
\hline Thru_Hole_5 & Input_Housing & $A F A \_f C 13$ & $A F A R \_f c 13$ \\
\hline Thru_Hole_6 & Input_Housing & AFA_fc13 & $A F A R \_f c 13$ \\
\hline Thru_Hole_7 & Input_Housing & AFA_fc13 & $A F A R \_f c 13$ \\
\hline Thru_Hole_8 & Input_Housing & AFA_fc13 & $A F A R \_f c 13$ \\
\hline
\end{tabular}

Table 12 OAM Features: asserted instances

\subsubsection{Asserted ArtifactAssociation Instances and Properties}

An Assembly can be composed by several Parts and the simple enumeration of them is represented through the properties artifactHasPart and artifactHasPart_direct. The class ArtifactAssociation and its subclasses (Connection, PositionOrientation and RelativeMotion) are used to represent the relationship between the Parts that are connected for creating an Assembly. The class Connection further has three subclasses FixedConnections, IntermittentConnections and MovableConnections.

For example, as an instance of Assembly, Ring_Gear_Assembly is composed by the parts Ring_Gear, Ring_Gear_Pin_1and Ring_Gear_Pin_2. This information does not provide any information on the relation between these parts. However, the two instances of the class FixedConnection (sub-class of ArtifactAssociation) $f C \_5$ and $f c \_6$ represents the real assembly configuration. The instance $f c \_5$ links the Ring_Gear and the Ring_Gear_Pin_1 and fc_6 links the Ring_Gear and the Ring_Gear_Pin_2. In this way it is possible to fully represent the Ring_Gear_Assembly structure.

In the FixedConnection class, only the relations between its instances and the parts linked to it have to be asserted. The other properties will be inferred by the reasoner through the SWRL rules.

The asserted instances and properties are listed in Table 13. For every instance the subclass of pertinence is specified through the name (fc:FixedConnection mc:MovableConnection po:PositionOrientation). 


\begin{tabular}{|c|c|}
\hline & Asserted Properties \\
\hline $\begin{array}{l}\text { ArtifactAssociation } \\
\text { Instances }\end{array}$ & $\begin{array}{l}\text { artifactAssociation2Part } \\
\text { (D:AA R:Part) }\end{array}$ \\
\hline$f c 1$ & Washer \\
\hline$f c 2$ & Output_Housing \\
\hline$f c 2$ & Bearing_1 \\
\hline fc3 & Output_Housing \\
\hline$f c 3$ & Bearing_2 \\
\hline$f c 5$ & Ring_Gear_Pin_1 \\
\hline$f c 5$ & Ring_Gear \\
\hline$f c 6$ & Ring_Gear_Pin_2 \\
\hline$f c 6$ & Ring_Gear \\
\hline$f c 7$ & Planet_Gear_1 \\
\hline$f c 7$ & Output_Shaft \\
\hline$f c 8$ & Planet_Gear_Pin_2 \\
\hline$f c 8$ & Output_Shaft \\
\hline$f c 9$ & Planet_Gear_Pin_3 \\
\hline fc9 & Output_Shaft \\
\hline fc10 & Ring_Gear_Pin_1 \\
\hline fc10 & Ring_Gear_Pin_2 \\
\hline fc10 & Output_Housing \\
\hline$f c 10$ & Output_Housing \\
\hline$f c 11$ & Screw_1 \\
\hline$f c 11$ & Screw_2 \\
\hline$f c 11$ & Screw_3 \\
\hline$f c 11$ & Screw_4 \\
\hline fc11 & Ring_Gear \\
\hline$f c 11$ & Ring_Gear \\
\hline$f c 11$ & Ring_Gear \\
\hline$f c 11$ & Ring_Gear \\
\hline$f c 11$ & Output_Housing \\
\hline$f c 11$ & Output_Housing \\
\hline fc11 & Output_Housing \\
\hline$f c 11$ & Output_Housing \\
\hline$f c 12$ & Ring_Gear \\
\hline fc12 & Input_Housing \\
\hline Fc13 & Screw_5 \\
\hline
\end{tabular}

\begin{tabular}{|c|c|}
\hline & Asserted Properties \\
\hline $\begin{array}{l}\text { ArtifactAssociation } \\
\text { Instances }\end{array}$ & $\begin{array}{l}\text { artifactAssociation2Part } \\
\text { (D:AA R:Part) }\end{array}$ \\
\hline fc13 & Screw_7 \\
\hline$f c 13$ & Screw_8 \\
\hline fc13 & Ring_Gear \\
\hline fc13 & Ring_Gear \\
\hline fc13 & Ring_Gear \\
\hline$f c 13$ & Ring_Gear \\
\hline fc13 & Input_Housing \\
\hline$f_{c 13}$ & Input_Housing \\
\hline fc13 & Input_Housing \\
\hline fc13 & Input_Housing \\
\hline$m c 1$ & Ring_Gear_Pin_1 \\
\hline$m c 1$ & Ring_Gear_Pin_2 \\
\hline$m c 2$ & Planet_Gear_Pin_2 \\
\hline$m c 2$ & Planet_Gear_2 \\
\hline$m c 3$ & Planet_Gear_Pin_3 \\
\hline$m c 3$ & Planet_Gear_3 \\
\hline$m c 4$ & Output_Shaft \\
\hline$m c 4$ & Bearing_1 \\
\hline mc4 & Bearing_2 \\
\hline$m c 5$ & Sungear \\
\hline$m c 5$ & Planet_Gear_1 \\
\hline$m c 6$ & Sungear \\
\hline$m c 6$ & Planet_Gear_2 \\
\hline$m c 7$ & Sungear \\
\hline$m c 7$ & Planet_Gear_3 \\
\hline$m c 9$ & Ring_Gear \\
\hline$m c 9$ & Planet_Gear_1 \\
\hline mc10 & Ring_Gear \\
\hline mc10 & Planet_Gear_2 \\
\hline$m c 11$ & Ring_Gear \\
\hline$m c 11$ & Planet_Gear_3 \\
\hline Po1 & Output_Shaft \\
\hline po1 & Sungear \\
\hline
\end{tabular}

Table 13 ArtifactAssociation: asserted instances and properties

\subsubsection{Asserted AssemblyFeatureAssociation Instances and Properties}

The AssemblyFeatureAssociation class has the same aim of ArtifactAssociation but at the feature level. If two parts are connected through an instance of ArtifactAssociation (e.g. $f c_{-} 1$ ) then two Features of these parts have to be connected through an instance of AssemblyFeatureAssociation (e.g., $A F A \_f c 1$ ). This class has two properties AFA2Feature and AFA 2 AFAR. The property AFA2Feature has the similar function as ArtifactAssociation2 Part and links at least two Features realizing an assembly constituted of two parts. The property AFA_2_AFAR links the instances of AssemblyFeatureAssociation with AssemblyFeatureAssociationRepresentation. 
The AssemblyFeatureAssociationRepresentation class is used to connect the Features with several classes used in tolerances and geometric representations. The asserted instances and properties are shown in Table 14.

\begin{tabular}{|c|c|c|}
\hline \multirow[b]{2}{*}{ AFA Instances } & \multicolumn{2}{|c|}{ Asserted Properties } \\
\hline & $\begin{array}{l}\text { AFA_2_AFAR } \\
\text { (D:AFA R:AFAR) }\end{array}$ & $\begin{array}{l}\text { AFA2Feature } \\
\text { (D:AFA R:Feature) }\end{array}$ \\
\hline \multirow{2}{*}{ AFA_fc1 } & \multirow{2}{*}{ AFAR_fc1 } & Groove \\
\hline & & Outer_Rim \\
\hline \multirow{2}{*}{ AFA_fc2 } & \multirow{2}{*}{ AFAR_fc2 } & Bearing_Seat_1 \\
\hline & & Outer_Race_1 \\
\hline \multirow{2}{*}{ AFA_fc3 } & \multirow{2}{*}{ AFAR_fc3 } & Bearing_Seat_2 \\
\hline & & Outer_Race_2 \\
\hline \multirow{4}{*}{ AFA_fc5 } & \multirow{4}{*}{ AFAR_fC5 } & pin_Cylinder_1 \\
\hline & & pin_Hole_1 \\
\hline & & pin_Cylinder_2 \\
\hline & & pin_Hole_2 \\
\hline \multirow{2}{*}{ AFA_fc7 } & \multirow{2}{*}{ AFAR_fc7 } & pin_Cylinder_3 \\
\hline & & pin_Hole_3 \\
\hline \multirow{2}{*}{ AFA_fC8 } & \multirow{2}{*}{ AFAR_fC8 } & pin_Cylinder_4 \\
\hline & & pin_Hole_4 \\
\hline \multirow{2}{*}{ AFA_fc9 } & \multirow{2}{*}{ AFAR_fc9 } & pin_Cylinder_5 \\
\hline & & pin_Hole_5 \\
\hline \multirow{4}{*}{ AFA_fc10 } & \multirow{4}{*}{ AFAR_fc10 } & pin_Cylinder_9 \\
\hline & & pin_Cylinder_10 \\
\hline & & pin_Hole_9 \\
\hline & & pin_Hole_10 \\
\hline \multirow{12}{*}{ AFA_fc11 } & \multirow{12}{*}{ AFAR_fc11 } & thread_1 \\
\hline & & thread_2 \\
\hline & & thread_3 \\
\hline & & thread_4 \\
\hline & & threaded_Hole_1 \\
\hline & & threaded_Hole_2 \\
\hline & & threaded_Hole_3 \\
\hline & & threaded_Hole_4 \\
\hline & & Thru_Hole_1 \\
\hline & & Thru_Hole_2 \\
\hline & & Thru_Hole_3 \\
\hline & & Thru_Hole_4 \\
\hline AFA_fc12 & AFAR_fc12 & Ring_Gear_Side \\
\hline
\end{tabular}




\begin{tabular}{|c|c|c|}
\hline & & Stepped_Side \\
\hline \multirow{12}{*}{ AFA_fc13 } & \multirow{12}{*}{ AFAR_fc13 } & thread_5 \\
\hline & & thread_6 \\
\hline & & thread_7 \\
\hline & & thread_8 \\
\hline & & threaded_Hole_1 \\
\hline & & threaded_Hole_2 \\
\hline & & threaded_Hole_3 \\
\hline & & threaded_Hole_4 \\
\hline & & Thru_Hole_5 \\
\hline & & Thru_Hole_6 \\
\hline & & Thru_Hole_7 \\
\hline & & Thru_Hole_8 \\
\hline \multirow{2}{*}{ AFA_mc1 } & \multirow{2}{*}{ AFAR_mc1 } & pin_Cylinder_6 \\
\hline & & Pin_Hole_6 \\
\hline \multirow{2}{*}{ AFA_mc2 } & \multirow{2}{*}{ AFAR_mc2 } & pin_Cylinder_7 \\
\hline & & pin_Hole_7 \\
\hline \multirow{2}{*}{ AFA_mc3 } & \multirow{2}{*}{ AFAR_mc3 } & pin_Cylinder_8 \\
\hline & & pin_Hole_8 \\
\hline \multirow{3}{*}{ AFA_mc4 } & \multirow{3}{*}{ AFAR_mc4 } & Bearing_Seat_3 \\
\hline & & Inner_Race_1 \\
\hline & & Inner_Race_2 \\
\hline \multirow{2}{*}{ AFA_mc5 } & \multirow{2}{*}{ AFAR_mc5 } & teeth_1 \\
\hline & & teeth_7 \\
\hline \multirow{2}{*}{ AFA_mc6 } & \multirow{2}{*}{ AFAR_mc6 } & teeth_2 \\
\hline & & teeth_9 \\
\hline \multirow{2}{*}{ AFA_mc7 } & \multirow{2}{*}{ AFAR_mc7 } & teeth_3 \\
\hline & & teeth_11 \\
\hline \multirow{2}{*}{ AFA_mc9 } & \multirow{2}{*}{ AFAR_mc9 } & teeth_4 \\
\hline & & teeth_8 \\
\hline \multirow{2}{*}{ AFA_mc10 } & \multirow{2}{*}{ AFAR_mc10 } & teeth_5 \\
\hline & & teeth_10 \\
\hline \multirow{2}{*}{ AFA_mc11 } & \multirow{2}{*}{ AFAR_mc11 } & teeth_6 \\
\hline & & teeth_12 \\
\hline \multirow{2}{*}{ AFA_po1 } & \multirow{2}{*}{ AFAR_po1 } & Output_Shaft_Feature \\
\hline & & Sun_Gear_Feature \\
\hline
\end{tabular}

Table 14 AssemblyFeatureAssociation: asserted instances and properties 


\subsubsection{Asserted AssemblyFeatureAssociationRepresentation Instances and Properties}

This class is used to link the Features with the geometric representation and tolerance specifications. For the correct connection between the Features and the detailed information cited before, two properties are specified for this class: AFAR_2_AFA and AFAR 2 Feature. The first has to be asserted. The second will be inferred. (see Table $15)$.

\begin{tabular}{|c|c|c|c|}
\hline & Asserted Properties & & Asserted Properties \\
\hline AFAR Instances & $\begin{array}{l}\text { AFAR_2_AFA } \\
\text { (D:AFAR R:AFA) }\end{array}$ & AFAR Instances & $\begin{array}{l}\text { AFAR_2_AFA } \\
\text { (D:AFAR R:AFA) }\end{array}$ \\
\hline AFAR_fc1 & AFA_fc1 & AFAR_mc1 & AFA_mc1 \\
\hline AFAR_fc2 & AFA_fc2 & AFAR_mc2 & AFA_mc2 \\
\hline AFAR_fc3 & AFA_fc3 & AFAR_mc3 & AFA_mc3 \\
\hline AFAR_fc5 & AFA_fc5 & AFAR_mc4 & AFA_mc4 \\
\hline AFAR_fc7 & AFA_fc7 & AFAR_mc5 & AFA_mc5 \\
\hline AFAR_fc8 & AFA_fc8 & AFAR_mc6 & AFA_mc6 \\
\hline AFAR_fc 9 & AFA_fc9 & AFAR_mc7 & AFA_mc7 \\
\hline AFAR_fc10 & AFA_fc10 & AFAR_mc9 & AFA_mc9 \\
\hline AFAR_fc11 & AFA_fc11 & AFAR_mc10 & AFA_mc10 \\
\hline AFAR_fc12 & AFA_fc12 & AFAR_mc11 & AFA_mc11 \\
\hline AFAR_fc13 & AFA_fc13 & AFAR_po1 & AFA_po1 \\
\hline
\end{tabular}

Table 15 AssemblyFeatureAssociationRepresentation: asserted instances and properties

\subsubsection{Inferred Instances and Properties}

In this section the output instances of each class will be presented. The term output instances refer to the instances that are inferred with the reasoning software (RACER or Jess) and the SWRL rules.

\subsubsection{Inferred Artifact Properties}

After the reasoning with RACER and Jess the input instances of Artifact are inferred (thanks to RACER) as instances of the class Assembly. For details see the following section.

\subsubsection{Inferred Assembly Properties}

After reasoning, the class Assembly is not empty anymore (see Table 16). The reasoning performed by RACER on the restriction defined for this class on the property 
artifactHasPart_direct min 2 has inferred the instances asserted in the class Artifact as elements of the class Assembly.

The Jess reasoning based on the SWRL (see section 5.2 for more details) rules has inferred not only the parts that constitute every instance of Assembly but also its related instances of ArtifactAssociation (see 6.2.1.1 for details on the reflexive definition of Output_Housing_Assembly).

\begin{tabular}{|c|c|c|}
\hline & \multicolumn{2}{|c|}{ Inferred Properties } \\
\hline Assembly Instances & $\begin{array}{l}\text { artifactHasPart } \\
\text { (D:Artifact R:Artifact) }\end{array}$ & $\begin{array}{l}\text { assembly2ArtifactAssociation } \\
\text { (D:Assembly } \\
\text { R:ArtifactAssociation) }\end{array}$ \\
\hline Output_Housing_Assembly & $\begin{array}{l}\text { Bearing_1 } \\
\text { Bearing_2 } \\
\text { Output_Housing } \\
\text { Washer } \\
\text { Output_Housing_Assembly* }\end{array}$ & $\begin{array}{l}\text { fc_1 } \\
\text { fc_2 } \\
\text { fc_3 } \\
\text { mc_4 }\end{array}$ \\
\hline Planet_Carrier_Assembly & $\begin{array}{l}\text { Output_Shaft } \\
\text { Planet_Gear_Pin_1 } \\
\text { Planet_Gear_Pin_2 } \\
\text { Planet_Gear_Pin_3 }\end{array}$ & $\begin{array}{l}\text { fc_-7 } \\
\text { fc_8 } \\
\text { fc_9 }\end{array}$ \\
\hline Planet_Gear_Carrier_Assembly & $\begin{array}{l}\text { Planet_Gear_1 } \\
\text { Planet_Gear_2 } \\
\text { Planet_Gear_3 } \\
\text { Planet_Carrier_Assembly }\end{array}$ & $\begin{array}{l}\mathrm{mc} \_1 \\
\mathrm{mc} \text {-2 } \\
\mathrm{mc} \text {-3 }\end{array}$ \\
\hline Ring_Gear_Assembly & $\begin{array}{l}\text { Ring_Gear } \\
\text { Ring_Gear_Pin_1 } \\
\text { Ring_Gear_Pin_2 }\end{array}$ & $\begin{array}{l}\text { fc_6 } \\
\text { fc_10 }\end{array}$ \\
\hline Planetary_Gear_System_Assembly & $\begin{array}{l}\text { Bearing_1 } \\
\text { Bearing_2 } \\
\text { Output_Housing } \\
\text { Washer } \\
\text { Output_Shaft } \\
\text { Planet_Gear_Pin_1 } \\
\text { Planet_Gear_Pin_2 } \\
\text { Planet_Gear_Pin_3 } \\
\text { Planet_Gear_1 } \\
\text { Planet_Gear_2 } \\
\text { Planet_Gear_3 } \\
\text { Ring_Gear } \\
\text { Ring_Gear_Pin_1 } \\
\text { Ring_Gear_Pin_2 } \\
\text { Sungear } \\
\text { Input_Housing } \\
\text { Screw_1 } \\
\text { Screw_2 } \\
\text { Screw_3 } \\
\text { Screw_4 } \\
\text { Screw_5 } \\
\text { Screw_6 } \\
\text { Screw_7 }\end{array}$ & $\begin{array}{l}\text { mc_1 } \\
\text { mc_2 } \\
\text { mc_3 } \\
\text { mc_4 } \\
\text { mc_5 } \\
\text { mc_6 } \\
\text { mc_7 } \\
\text { mc_9 } \\
\text { mc_10 } \\
\text { mc_11 } \\
\text { po_1 } \\
\text { fc_5 } \\
\text { fc_7 } \\
\text { fc_8 } \\
\text { fc_9 } \\
\text { fc_10 } \\
\text { fc_11 } \\
\text { fc_12 } \\
\text { fc_13 }\end{array}$ \\
\hline
\end{tabular}


Screw_8

Table 16 Assembly inferred properties

\subsubsection{Inferred Meaning_Less_Artifact Instances}

After the Jess reasoning, the Meaning_Less_Artifact class (see Section 5.2.1.3) is no longer empty. Two instances of the class Artifact are reclassified as not well defined. In Table 17 the reclassified instances are presented.

\begin{tabular}{|l|}
\hline Meaning_Less_Artifact Instances \\
\hline Output_Housing_Assembly \\
\hline Planetary_Gear_System_Assembly \\
\hline
\end{tabular}

Table 17 Meaning_Less_Artifact inferred instances

As expected, the instance (Output_Housing_Assembly) with a self reference (inadmissible in assembly representation) is reclassified as element of this class. Also notice that the Planetary_Gear_System_Assembly is reclassified to this class since the inadmissible Output_Housing_Assembly is a sub-assembly of the Planetary_Gear_System_Assembly.

\subsubsection{Inferred Part Properties}

After the Jess engine reasoning, the indirect property partofArtifact, inverse of the property artifactHaspart, is inferred for each instance. For example, the instance Bearing_1 is directly a part of the Output_Housing_Assembly, which in turn is a part of the Planetary_Gear_System_Assembly. The Bearing_1 is inferred both as part of the Output_Housing_Assembly and Planetary_Gear_System_Assembly. The inferred properties are presented in Table 18.

\begin{tabular}{|c|c|}
\hline & Inferred Instances \\
\hline Part Instances & $\begin{array}{l}\text { partOfArtifact } \\
\text { (D:Artifact R:Artifact) }\end{array}$ \\
\hline Bearing_1- 2 & $\begin{array}{l}\text { Output_Housing_Assembly } \\
\text { Planetary_Gear_System_Assembly }\end{array}$ \\
\hline Input Housing & Planetary_Gear_System_Assembly \\
\hline Output_Housing & $\begin{array}{l}\text { Output_Housing_Assembly } \\
\text { Planetary_Gear_System_Assembly }\end{array}$ \\
\hline Output_Shaft & $\begin{array}{l}\text { Planet_Carrier_Assembly } \\
\text { Planet_Gear_Carrier_Assembly } \\
\text { Planetary_Gear_System_Assembly }\end{array}$ \\
\hline Planet_Gear_1 - 3 & $\begin{array}{l}\text { Planet_Gear_Carrier_Assembly } \\
\text { Planetary_Gear_System_Assembly }\end{array}$ \\
\hline Planet_Gear_Pin_1-3 & $\begin{array}{l}\text { Planet_Carrier_Assembly } \\
\text { Planet_Gear_Carrier_Assembly } \\
\text { Planetary_Gear_System_Assembly }\end{array}$ \\
\hline
\end{tabular}




\begin{tabular}{|l|l|}
\hline Ring_Gear & $\begin{array}{l}\text { Ring_Gear_Assembly } \\
\text { Planetary_Gear_System_Assembly }\end{array}$ \\
\hline Ring_Gear_Pin_1- 2 & $\begin{array}{l}\text { Ring_Gear_Assembly } \\
\text { Planetary_Gear_System_Assembly }\end{array}$ \\
\hline Screw_1-Screw 8 & Planetary_Gear_System_Assembly \\
\hline Sungear & Planetary_Gear_System_Assembly \\
\hline Washer & $\begin{array}{l}\text { Output_Housing_Assembly } \\
\text { Planetary_Gear_System_Assembly }\end{array}$ \\
\hline
\end{tabular}

Table 18 Part: inferred properties

\subsubsection{Inferred Feature Instances and Properties}

The class Feature has to be completely defined from the beginning. As this is the lowest level of the representation, it is not possible to infer anything about the Features from the structure of the Assemblies.

\subsubsection{Inferred ArtifactAssociation Properties}

With the SWRL rules the Jess reasoning engine is able to infer the properties: artifactAssociation2Assembly and artifactAssociation2AssemblyFeatureAssociation. The first is the inverse of the property assembly2ArtifactAssociation inferred for the class Assembly. The second is the property that links the instances of this class with the ones of the class AssemblyFeatureAssociation that has the same rules of the class ArtifactAssociation but on the features level (Table 19).

\begin{tabular}{|l|l|l|}
\hline & \multicolumn{2}{|c|}{ Inferred Properties } \\
\hline \multirow{2}{*}{$\begin{array}{l}\text { ArtifactAssociation } \\
\text { Instancies }\end{array}$} & $\begin{array}{l}\text { artifactAssociation2Assembly } \\
\text { (D:Artifact R:Assembly) }\end{array}$ & $\begin{array}{l}\text { artifactAssociation2AFA } \\
\text { (D:ArtifactAssociation } \\
\text { R:AssemblyFeatureAssociation) }\end{array}$ \\
\hline \multirow{2}{*}{ fc_1 } & Output_Housing_Assembly & AFA_fc1 \\
\hline fc_2 & Output_Housing_Assembly & AFA_fc2 \\
\hline fc_3 & Output_Housing_Assembly & AFA_fc3 \\
\hline fc_3 & Output_Housing_Assembly & AFA_fc3 \\
\hline fc_5 & Planetary_Gear_System_Assembly & AFA_fc5 \\
\hline fc_6 & Ring_Gear_Assembly & AFA_fc6 \\
\hline fc_7 & Planet_Carrier_Assembly & AFA_fc7 \\
\hline fc_8 & Planet_Carrier_Assembly & AFA_fc8 \\
\hline fc_9 & Planet_Carrier_Assembly & AFA_fc9 \\
\hline \multirow{2}{*}{ fc_10 } & Ring_Gear_Assembly & AFA_fc10 \\
\hline fc_11 & Planetary_Gear_System_Assembly & AFA_fc11 \\
\hline fc_12 & Planetary_Gear_System_Assembly & AFA_fc12 \\
\cline { 3 - 3 } & AFA_fc13 \\
\hline
\end{tabular}




\begin{tabular}{|l|l|l|} 
& & \\
fc_13 & Planetary_Gear_System_Assembly & AFA_fc12 \\
\cline { 3 - 3 } & & AFA_fc13 \\
\hline mc_1 & Planet_Gear_Carrier_Assembly & AFA_mc1 \\
\hline mc_3 & Planet_Carrier_Assembly & AFA_mc2 \\
\hline mc_4 & Planet_Gear_Carrier_Assembly & AFA_mc3 \\
\hline mc_5 & Output_Housing_Assembly & AFA_mc4 \\
\hline mc_6 & Planetary_Gear_System_Assembly & AFA_mc5 \\
\hline mc_7 & Planetary_Gear_System_Assembly & AFA_mc6 \\
\hline mc_9 & Planetary_Gear_System_Assembly & AFA_mc7 \\
\hline mc_10 & Ring_Gear_Assembly & AFA_mc9 \\
\hline mc_10 & Planet_Gear_Carrier_Assembly & AFA_mc10 \\
\hline mc_11 & Planet_Gear_Carrier_Assembly & AFA_mc10 \\
\hline po_1 & Planet_Gear_Carrier_Assembly & AFA_mc11 \\
\hline po_1 & Planetary_Gear_System_Assembly & AFA_po1 \\
\hline
\end{tabular}

Table 19 ArtifactAssociation: inferred properties

\subsubsection{Inferred AssemblyFeatureAssociation Properties}

After the reasoning, the property between the two classes AssemblyFeatureAssociation and ArtifactAssociation is inferred (AssemblyFeatureAssociation2ArtifactAssociation). This is possible with the Jess engine and SWRL rules (see section 5.2 for details). Rules form the relationships between parts and features, between parts and artifacts associations and between features. The class AssemblyFeatureAssociation permits the deduction of the property (see Table 20).

\begin{tabular}{|l|l|}
\hline & \multicolumn{1}{|c|}{ Inferred Properties } \\
\hline AFA Instances & $\begin{array}{l}\text { AssemblyFeatureAssociation2ArtifactAssociation } \\
\text { (D:AFA R:ArtifactAssociation) }\end{array}$ \\
\hline AFA_fc1 & fc_1 \\
\hline AFA_fc2 & fc_2 \\
\hline AFA_fc3 & fc_3 \\
\hline AFA_fc5 & fc_5 \\
\hline AFA_fc7 & fc_7 \\
\hline AFA_fc8 & fc_8 \\
\hline AFA_fc9 & fc_9 \\
\hline AFA_fc10 & fc_10 \\
\hline AFA_fc11 & fc_11 \\
\hline AFA_fc12 & fc_12 \\
\hline
\end{tabular}




\begin{tabular}{|l|l|} 
AFA_fc13 & fc_13 \\
\hline AFA_mc1 & mc_1 \\
\hline AFA_mc2 & mc_2 \\
\hline AFA_mc3 & mc_3 \\
\hline AFA_mc4 & mc_4 \\
\hline AFA_mc5 & $m c \_5$ \\
\hline AFA_mc6 & mc_6 \\
\hline AFA_mc7 & mc_7 \\
\hline AFA_mc9 & mc_9 \\
\hline AFA_mc10 & mc_10 \\
\hline AFA_mc11 & mc_11 \\
\hline AFA_po1 & po_1 \\
\hline
\end{tabular}

Table 20 AssemblyFeatureAssociation: inferred property

\subsubsection{Inferred AssemblyFeatureAssociationRepresentation Properties}

The inferred property is AFAR_2_Feature. From the asserted properties that links the class AssemblyFeatureAssociationRepresentation to AssemblyFeatureAssociation and AssemblyFeatureAssociation to Feature, the reasoner is able to infer the property AFAR_2_Feature. The detailed inferred instances are presented in Table 21.

\begin{tabular}{|c|c|c|c|}
\hline & Inferred Properties & & Inferred Properties \\
\hline $\begin{array}{l}\text { AFAR } \\
\text { Instances }\end{array}$ & $\begin{array}{l}\text { AFAR_2_Feature } \\
\text { (D:AFAR R:Feature) }\end{array}$ & $\begin{array}{l}\text { AFAR } \\
\text { Instances }\end{array}$ & $\begin{array}{l}\text { AFAR_2_Feature } \\
\text { (D:AFAR R:Feature) }\end{array}$ \\
\hline \multirow{2}{*}{ AFAR_fc1 } & Groove & \multirow{12}{*}{ AFAR_fc13 } & thread_5 \\
\hline & Outer_Rim & & thread_6 \\
\hline \multirow{2}{*}{ AFAR_fc2 } & Bearing_Seat_1 & & thread_7 \\
\hline & Outer_Race_1 & & thread_8 \\
\hline \multirow{2}{*}{ AFAR_fc3 } & Bearing_Seat_2 & & threaded_Hole_1 \\
\hline & Outer_Race_2 & & threaded_Hole_2 \\
\hline \multirow{4}{*}{ AFAR_fc5 } & pin_Cylinder_1 & & threaded_Hole_3 \\
\hline & pin_Hole_1 & & threaded_Hole_4 \\
\hline & pin_Cylinder_2 & & Thru_Hole_5 \\
\hline & pin_Hole_2 & & Thru_Hole_6 \\
\hline \multirow{2}{*}{ AFAR_fc7 } & pin_Cylinder_3 & & Thru_Hole_7 \\
\hline & pin_Hole_3 & & Thru_Hole_8 \\
\hline \multirow{2}{*}{ AFAR_fc8 } & pin_Cylinder_4 & \multirow{2}{*}{ AFAR_mc1 } & pin_Cylinder_6 \\
\hline & pin_Hole_4 & & Pin_Hole_6 \\
\hline \multirow{2}{*}{ AFAR_fc9 } & pin_Cylinder_5 & \multirow{2}{*}{ AFAR_mc2 } & pin_Cylinder_7 \\
\hline & pin_Hole_5 & & pin_Hole_7 \\
\hline
\end{tabular}




\begin{tabular}{|c|c|c|c|}
\hline \multirow{4}{*}{ AFAR_fc10 } & pin_Cylinder_9 & \multirow{2}{*}{ AFAR_mc3 } & |pin_Cylinder_8 \\
\hline & pin_Cylinder_10 & & pin_Hole_8 \\
\hline & pin_Hole_9 & \multirow{3}{*}{ AFAR_mc4 } & Bearing_Seat_3 \\
\hline & pin_Hole_10 & & Inner_Race_1 \\
\hline \multirow{12}{*}{ AFAR_fc11 } & thread_1 & & Inner_Race_2 \\
\hline & thread_2 & \multirow{2}{*}{ AFAR_mc5 } & teeth_1 \\
\hline & thread_3 & & teeth_7 \\
\hline & thread_4 & \multirow{2}{*}{ AFAR_mc6 } & teeth_2 \\
\hline & threaded_Hole_1 & & teeth_9 \\
\hline & threaded_Hole_2 & \multirow{2}{*}{ AFAR_mc7 } & teeth_3 \\
\hline & threaded_Hole_3 & & teeth_11 \\
\hline & threaded_Hole_4 & \multirow{2}{*}{ AFAR_mc9 } & teeth_4 \\
\hline & Thru_Hole_1 & & teeth_8 \\
\hline & Thru_Hole_2 & \multirow{2}{*}{ AFAR_mc10 } & teeth_5 \\
\hline & Thru_Hole_3 & & teeth_10 \\
\hline & Thru_Hole_4 & \multirow{2}{*}{ AFAR_mc11 } & teeth_6 \\
\hline \multirow{3}{*}{ AFAR_fc12 } & Ring_Gear_Side & & teeth_12 \\
\hline & Stepped_Side & \multirow{2}{*}{ AFAR_po1 } & Output_Shaft_Feature \\
\hline & & & Sun_Gear_Feature \\
\hline
\end{tabular}

Table 21 AssemblyFeatureAssociationRepresentation: inferred properties

\subsubsection{Kinematic Information Representation}

The Planetary Gear System is used for transmitting motion and for this reason the model has to be able to represent the relative motion of the single parts.

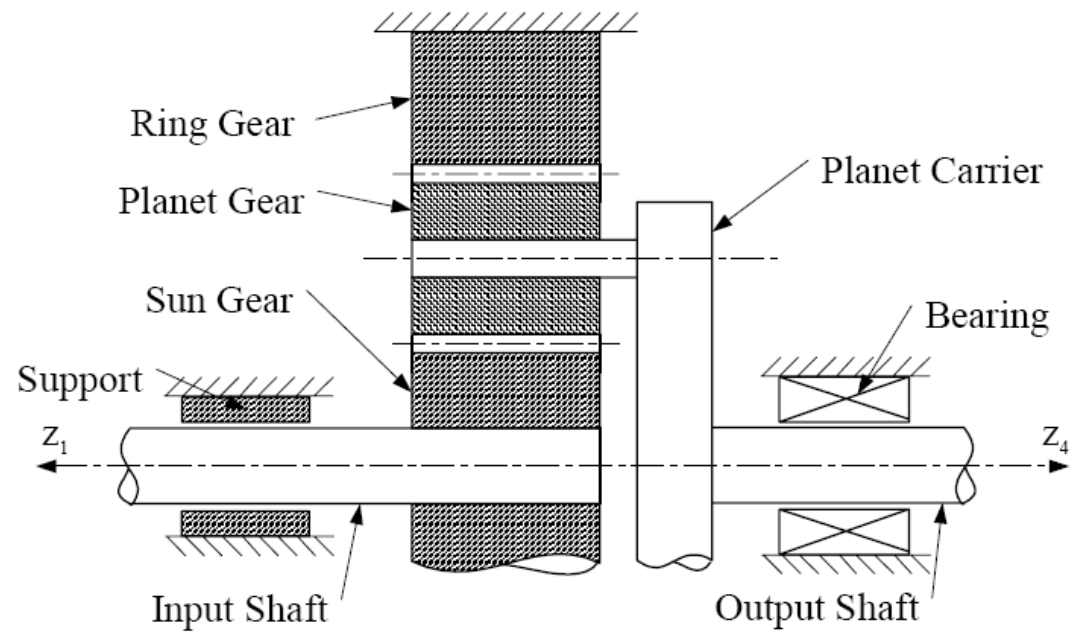

Figure 29 Kinematic Diagram of Planetary Gear System 
Figure 29 illustrates the Kinematic Diagram of Planetary Gear System. Table 22 presents the kinematic pairs and the associated parts that are identified from the planetary gear system. For convenience, numbers are used to distinguish the three planet gears and the kinematic pairs of the same type. As shown in Table 22, two types of kinematic pairs (GearPair and RevolutePair) are used in the planetary gear system.

\begin{tabular}{|l|l|}
\hline Kinematic Pairs & Associated Parts \\
\hline Revolute Pair 1 & Unknown Support - Sungear (Input Shaft) \\
\hline Gear Pair 1 & Sungear - Planet Gear 1 \\
\hline Gear Pair 2 & Sungear - Planet Gear 2 \\
\hline Gear Pair 3 & Sungear - Planet Gear 3 \\
\hline Gear Pair 4 & Planet Gear 1 - Ring Gear \\
\hline Gear Pair 5 & Planet Gear 2 - Ring Gear \\
\hline Gear Pair 6 & Planet Gear 3 - Ring Gear \\
\hline Revolute Pair 2 & Planet Gear 1 - Planet Carrier \\
\hline Revolute Pair 3 & Planet Gear 2 - Planet Carrier \\
\hline Revolute Pair 4 & Planet Gear 3 - Planet Carrier \\
\hline Revolute Pair 5 & Planet Carrier - Bearing \\
\hline
\end{tabular}

Table 22 Kinematic Pairs and Associated Parts of Planet Gear System

Assigning frames to each link (part) of the gear system is essential to describe the movements of each part. In general, two coordinate systems or frames are needed to describe the kinematic behavior of any KinematicPair, each attached to a link of the pair. Considering that a binary link is associated with two KinematicPairs (one with the preceding link and the other with the following link), there are two frames associated with the link. The KinematicPairs (instances of classes GearPair and RevolutePair) contain their specific kinematic information (constraints of the pair) to describe their own behavior and they are connected with the class AFAR through the property AFAR_2_KinematicPair and with the class Feature through the property feature_2_KinematicPair. It is also possible to represent the path of each movement with specific geometric characteristics through subclasses of the classes KinematicPair and KinematicPath. With this representation every movement could be represented in the OAM-OWL and thereby possible to perform reasoning between the classes KinematicPair, KinematicPath, AFAR and Feature.

\subsubsection{Tolerance Representation in the Planetary Gear System}

The tolerance schema is adopted from the OAM-UML, which is based on the standard ASME Y14.5 M [45]. For every type of tolerance defined in the standard, a class is defined to represent the tolerance value and other needed information. For example, consider the Sungear and its tolerances as in Figure 30. 


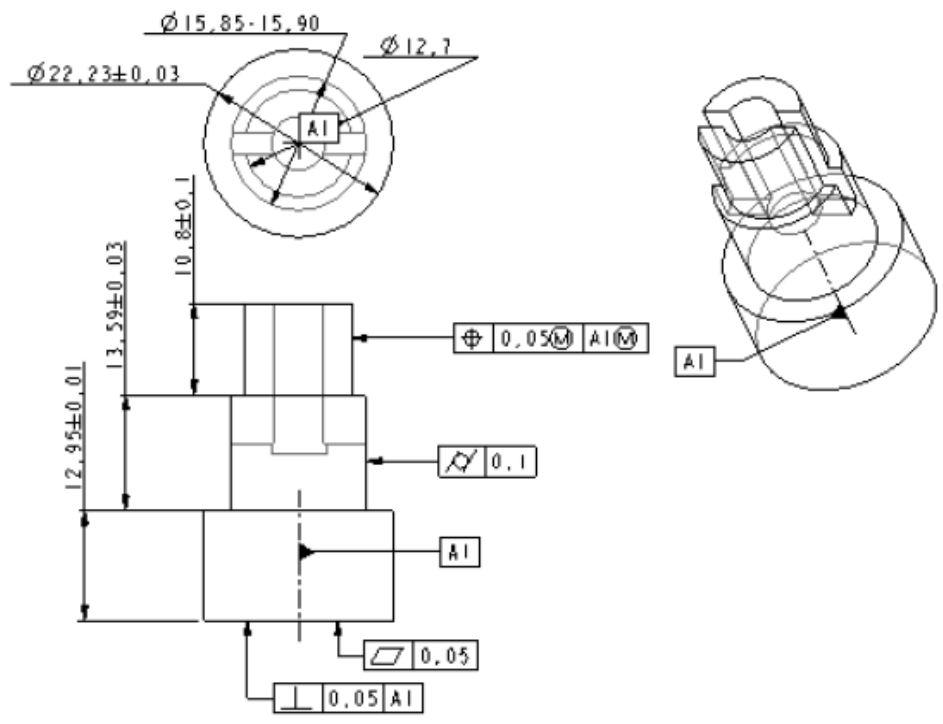

Figure 30 Sungear tolerances

Consider the highlighted Sun_Gear_Feature on the top of the Sungear. Notice that there are two different tolerances defined: a cylindricity tolerance and dimensional tolerance. This means that in the class Tolerance of the model two instances have to be created and linked through the property OAMFeature_toleranced_by (domain: feature range:Tolerance) to the Sun_Gear_Feature instance. To define a cylindricity tolerance, a reference axis is needed and with this aim a new feature has to be added to the Sungear: the DatumFeature_Axis_1. This particular instance is created in the class DatumFeature that is a subclass of OAMFeature. After setting DatumFeature_Axis_1 as reference for the cylindricity tolerance it is possible to define its attributes. The dimension of the tolerance is implemented as an instance with a numeric value of a dedicated class called Size. This choice is done to allow for reusing the same tolerance data when possible. For the dimensional tolerance the steps are the same but the creation of a datum reference is not needed.

\section{Results and Discussion}

Even if the interoperability between different systems is growing, the current PLM solutions are inefficient while screening data (usually in terabytes) clustered in companies. This necessitates a need for a data analysis system. This scenario is due to the inherent drawback with the commonly used approaches, to give any sort of meaning to the stored data to help systems to understand/react immediately to the kind of information saved in a particular cluster. This problem is present in any entity that collects great quantity of data (an entity could be anything from a whole organization, a single division or office). Generally every entity has good knowledge of the kind of data it manages (nevertheless may still need the assistance of a dedicated data analysis tool). However, this knowledge can become complex if we refer to different subjects of a supply chain or to a set of divisions or facilities trying to share data in a PLM context. The aim of this 
work, i.e., the development of an OWL version of the Open Assembly Model fits the above mentioned scenario. The underlying reasons for the creation of the OWL version of the assembly model are:

- A standard data structure developed directly in a Web-oriented language such as OWL: this assures the highest level of compatibility and diffusion.

- New reasoning capabilities offered by the ontological approach: OWL is developed with the intent of supporting the growth of the Semantic Web and offers the possibility to give to the data structure not only a format but a meaning intelligible by a computer. This allows the machines to reason this ontology to deduct knowledge and more information from the stored data.

The proposed OAM-OWL aims to address a data representation model for interoperability between software platforms with a capability of sharing meaningful stored data. In the subsequent Section 7.1 the novelty of the OAM model will be discussed along with a brief analysis of its capability. Section 7.2 presents a discussion on certain limitations scopes for future research.

\subsection{Model Advantages}

The first advantage of an ontological approach is the possibility to use the rich Vocabulary defined for this language. In the OWL model, it is possible to use classes and properties to define any kind of relation between different elements of representation. The property-based nature of OWL allows us to create all operations possible using sets. Concepts such as intersection and union can be used to tailor modeling activities based on the specific needs of the developer and recur to the definition of cardinality constraints if needed. The conceptual difference between UML and OWL offers the advantage to define on the same properties different constraints for different classes.

The structure and the semantic nature of the OWL model can be understood and reasoned by suitable reasoning software. In this application we deployed RACER to check the consistency of the developed ontology, to reclassify classes and to infer the related instances.

The consistency check capability of the reasoner is helpful for testing the correctness of the developed model and to find class definition or restrictions that could be contrasting. This is much more than a simple error check. The consistency check is aimed to test if the semantic (meaning) included in the model makes sense and not just the syntax.

The class reclassification is another interesting feature offered by RACER. Let's consider the previous example of the two-seat and four-seat cars with an added class car. The class car is defined as the class that contains all the elements with four wheels and with no precise number of passengers. If we add the condition (to be a car it has to have four wheels) to the two previous classes then the reasoner will reclassify the two- and fourseats cars as subclasses of the class car. 
RACER has the capability to infer instances of classes as elements of a different class that are of immediate relevance. Following the restrictions and the cardinality constraints defined in the model the reasoner is able to understand (infer) the particular kind of instance to be analyzed. This means that after the reclassification of the classes of the car in the earlier example, if an instance of car is created and is defined as a car with two seats, the reasoner will infer that in the ontology there is a two-seat car inserted.

The reasoning performed with RACER is useful for testing the consistency of the ontology and for relocating instances following the class definition. These are already good improvements to the previous versions of the models, but with the Jess engine it is possible to extract much more knowledge from the stored data. Jess is a rule engine that is able to process rules written with SWRL. With the combination of these two elements it is possible to define any rule on the representation and with the reasoning function Jess is able to add knowledge to the stored information. The level of complexity is higher than in the RACER reasoning. As with RACER, the inferred properties are directly related to the intrinsic structure of the ontology and hence limited to the comparison between the characteristics of any single instance and classes definitions. With Jess, the operation is performed on the ontology by the Jess engine through the SWRL rules and is more like a deduction then a simple comparison. Consider for example one of the reasonings performed in the implemented model to clarify this concept.

In the Planetary Gear Example, to create an assembly the connection between two parts is needed and it is represented by a relationship between two features of those parts. Only by specifying the relation between the two features of the two parts, the model is able to infer that the two parts have to be connected.

\subsection{Limitations and future research directions}

The OWL-OAM model has some criticisms, most of them inherent to the version of the language OWL 1.1 used and its integration with the tool Protégé-OWL. This can be attributed to the fact that both of them are still new and evolving. The final specification of OWL (1.1) was released in 2004 and Protégé is still available only in beta version. The first limitation of the current version of OWL 1.1 is the impossibility to define dynamic ranges for properties. Considering the example in Figure 31, it is impossible to specify that the range of the property $\mathrm{A} 2 \mathrm{C}$ has to be represented by the elements of $\mathrm{C}$ connected with elements of $\mathrm{B}$.

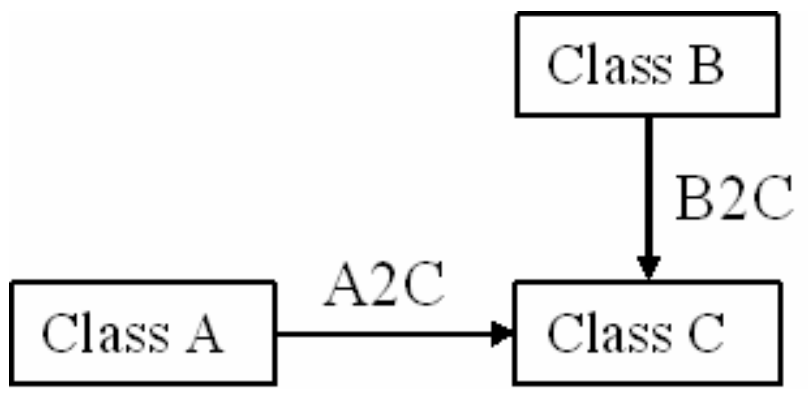

Figure 31 Dynamic Range Example 
This kind of structure could be useful to immediately identify (in large instantiated models) the instances of a class related with a particular instance.

Another limitation is related to the Open World Assumption present in the current version of OWL. The Open World Assumption makes it impossible to define an element as "is not an...". It is possible to define set operations for defining classes but, due to the Open World Assumption, it is not possible to define in a given set a subset and it's complement. In Figure 32 there exists a set with two subsets defined as partitions of the biggest set, but there still exists another element that is not a part of the two subsets.

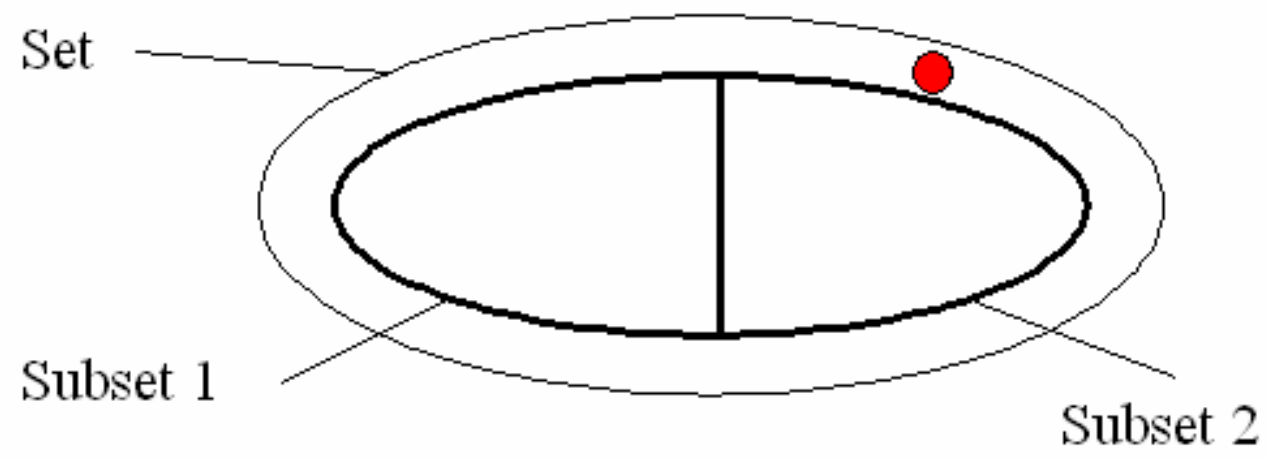

Figure 32 Open World Assumption

Considering the OAM-OWL class Artifact and its subclasses Assembly and Part, it is not possible to define as parts all the artifacts that are not assemblies. These instances will be considered as simple artifacts.

The previous problem could not be solved even with the SWRL rules. In fact, in the actual version of SWRL, logic operators like OR, NOT and XOR are not present. Although it is possible to infer that an Artifact created by many parts is an Assembly, it is impossible to infer that an Artifact not composed by parts is a Part.

Another problem is associated with the current version of Protégé-OWL. In fact it is not possible to accept inferred instances. Even if the tool recognizes that an Artifact created by many parts is an Assembly, it is not possible to specify some properties concerning only Assembly. This issue is expected to be solved with the next release of the tool.

All the open issues discussed above will be solved with the new release of OWL 2.0. Besides the issues, we can still appreciate the potentialities of the new ontology for the representation of the Open Assembly Model. This work can be considered an initial step in the standardization process within the PLM applications.

\section{Acknowledgements}

The authors wish to acknowledge the valuable comments, suggestions and improvements from Dr.Steven Fenves, Dr.Eswaran Subrahmanian and from all the researchers of the 
Design Process Group. Their comments have substantially improved and shaped the report.

\section{Disclaimer}

No approval or endorsement of any commercial product by NIST is intended or implied. Certain commercial equipment, instruments or materials are identified in this report to facilitate better understanding. Such identification does not imply recommendations or endorsement by NIST nor does it imply the materials or equipment identified are necessarily the best available for the purpose.

\section{References}

1. ISO. ISO 13584-1:2001, Industrial automation systems and integration -- Parts library -- Part 1: Overview and fundamental principles. 2001. ISO.

2. ISO. IEC 62264-2:2004, Enterprise-control system integration. 2004. ISO.

3. ISO 10303-1:1994, Industrial automation systems and integration -- Product data representation and exchange -- Part 1: Overview and fundamental principles.

4. Porter, M. How competitive forces shape strategy. 1979. Harvard business Review, Harvard business Review.

5. Product Life Cycle Support (PLCS), Frequently Asked Questions. http://xml.coverpages.org/PLCSInc-FAQv2-20030804.pdf . 8-4-2003.

6. OMG. UML 2.0: Infrastucture. 5-7-2005.

7. Fenves, S. J. A Core Product Model For Representing Design Information. NISTIR 6736. 2001. Gaithersburg, MD 20899, USA, National Institute of Standards and Technology.

8. Fenves, S, Foufou, S, Bock, C, Bouillon, N, and Sriram, R. D. CPM2: A Revised Core Product Model for Representing Design Information . NISTIR 7185. 2004. Gaithersburg, MD 20899, USA, National Institute of Standards and Technology.

9. Baysal, M. M, Roy, U, Sudarsan, R, Sriram, R. D, and Lyons, K. W. The Open Assembly Model for the Exchange of assembly and tolerance information: overview and example. 2004. Salt Lake City, Utah.

10. Web Ontology Language (OWL). http://www.w3.org/2004/OWL/ . 2005. 
11. Grady Booch, Jim Rumbaugh, and Ivar Jacobson. UML User Guide. 1999. Addison-Wesley.

12. J.Rumbaugh. The UML Reference Manual. 1999. Addison-Wesley.

13. G Mocko. A Knowledge Repository for Behavioral Models in Engineering Design. 2004. 24th ASME Computers and Information in Engineering.

14. M.Flower. UML distilled. 2004. Addison-Wesley.

15. Schenck D, Wilson PR. Information modeling: the EXPRESS way. New York: Oxford University Press, 1994.

16. ISO. www.step-nc.org.

17. STEP ISO 10303. http://www.steptools.com/library/standard/ . 2006. STEP Tools, Inc.

18. Feeney AB. The STEP Modular Architecture. Journal for Computing and Information Science in Engineering 2002;2:132-5.

19. Gruber, T. R. Towards principles for the design of ontologies used for knowledge sharing. 1993. Padova, Italy. International workshop on Formal Ontology.

20. Gruber, T. What is an Ontology? http://www-ksl.stanford.edu/kst/what-is-anontology.html . 1993.

21. G.Schreiber. OWL Web Ontology Language Reference. 2004. W3C.

22. Harmelen. Reviewing the design of DAML+OIL: An ontology language for the semantic web. 2001. Proc. of the 18th Nat. Conf. on Artificial Intelligence.

23. Patel-Schneider. Semantic web: ontology construction. 2006. International World Wide Web Conference.

24. RDF-Resource Description Framework. www.w3.org/RDF/ . 2006.

25. D.Brickley, R. V. Guha. Resource Description Framework (RDF) Model and Syntax Specification. 1999. W3C Recommendation submitted.

26. K Falkovych, M Sabou H Stuckenschmidt. UML for the Semantic Web: Transformation-Based Approaches. 2003.

27. T.Bittner, M. Donnelly B. Smith. Individuals, Unicersals, Collections: On the Foundational Relations of Ontology. 2005. Saarland University, Germany; University of Buffalo, NY.

28. E.Rosch. Principles of Categoization. 1978. Hillsdale. 
29. F.Giunchiglia, M. Marchese I. Zaihrayeu. Encoding Classifications into Lightweight Ontologies. 2005. Italy, Department of Information and Communication Technology, University of Trento.

30. Ontology Definition Metamodel Specification. Object Management Group.

31. D.Berardi, D.Calvanese G.De Giacomo. Reasoning on UML class diagrams.

32. J.Parsons, Yari Wand. Choosing Classes in Conceptual Modeling. Commuication of ACM 40(No. 6). 1997.

33. Evan Wallace and Natasha Noy. Simple part-whole relations in OWL Ontologies. 2005. W3C.

34. protégé Official Web Page. protege.stanford.edu . 2006.

35. H Knublauch, RW Fergerson NF Noy MA Musen. The Protégé OWL Plugin: An Open Development Environment for Semantic Web Applications. 2004. Third International Semantic Web Conference (ISWC 2004), 2004.

36. D.L.McGuinnes, J. Wright. Conceptual modelling for configuration: A description logic-based approach. Cambridge Journals . 1998.

37. D.L.McGuinnes. Description Logics Emerge from Ivory Towers. 2000. Stanford University.

38. RACER Reasoner. www.sts.tu-harburg.de/-r.f.moeller/racer . 2006.

39. SWRL plug-in for Protégé-OWL. protege.stanford.edu/plugins/owl/swrl . 2006.

40. SWRL: A Semantic Web Rule Language. www.w3.org/Submission/SWRL . 2006.

41. Jess Rule Engine. herzberg.ca.sandia.gov/jess . 2006.

42. P.Onions, G. Orange. A Model for Knowledge that supports Ontology and Epistemology. 2002. Leeds Metropolitan University, School of Information Management.

43. B.Smith. The Basic Tools of Formal Ontology. In: IOS Press. Formal Onthology in Information Systems. 1998:19-28.

44. B.Smith. Blackwell Guide to the Philosophy of Computing and Information: Chapter Ontology. 155-166. 2003. Oxford, Blackwell.

45. ASME. Dimensioning and Tolerancing Y14.5M. ASME, 1994. 


\section{Appendix}

\section{A few snapshots of the Model in Protégé OWL:}

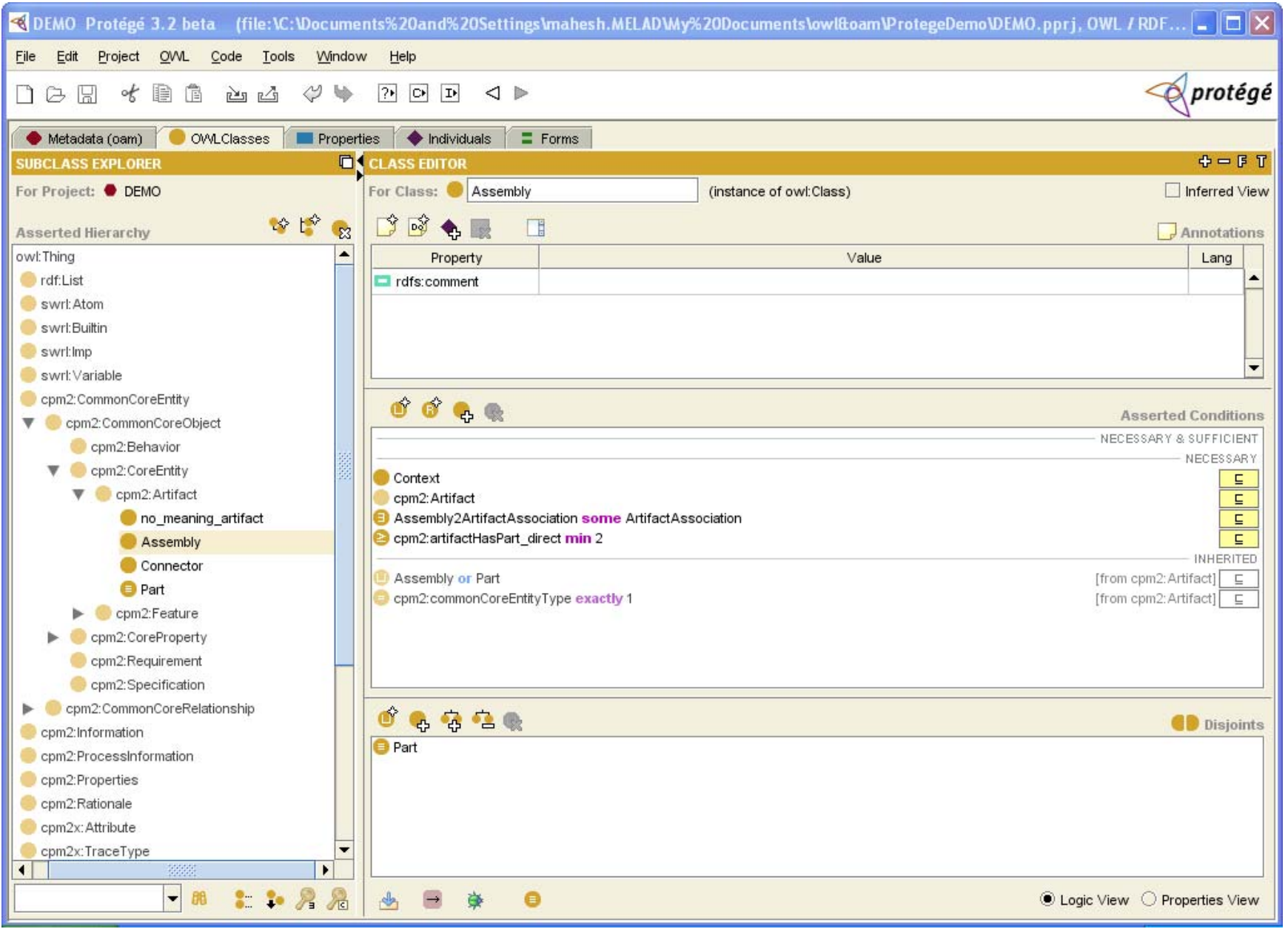

Snapshot A1 Subclass Explorer 


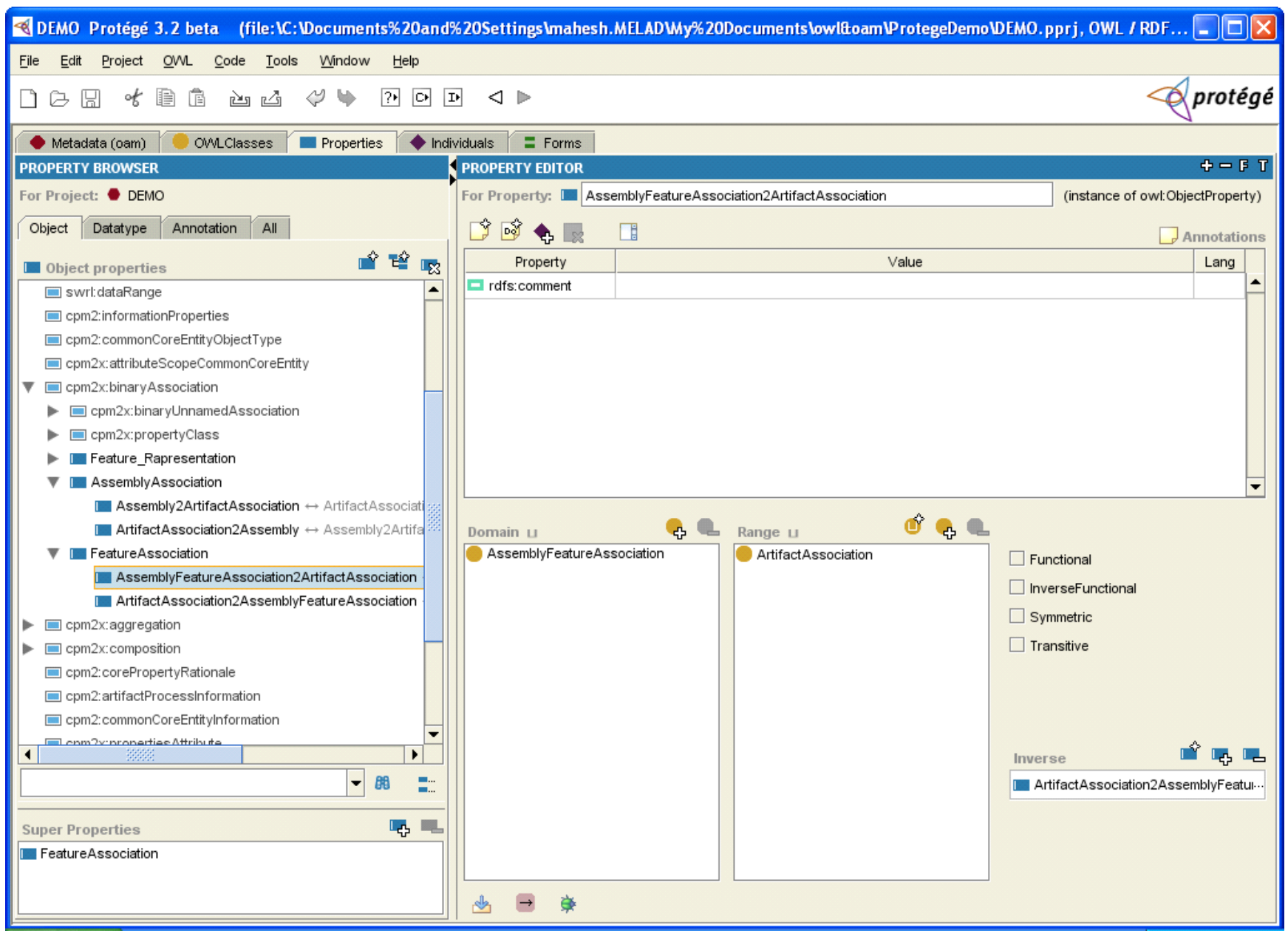

Snapshot A2 Property Browser 


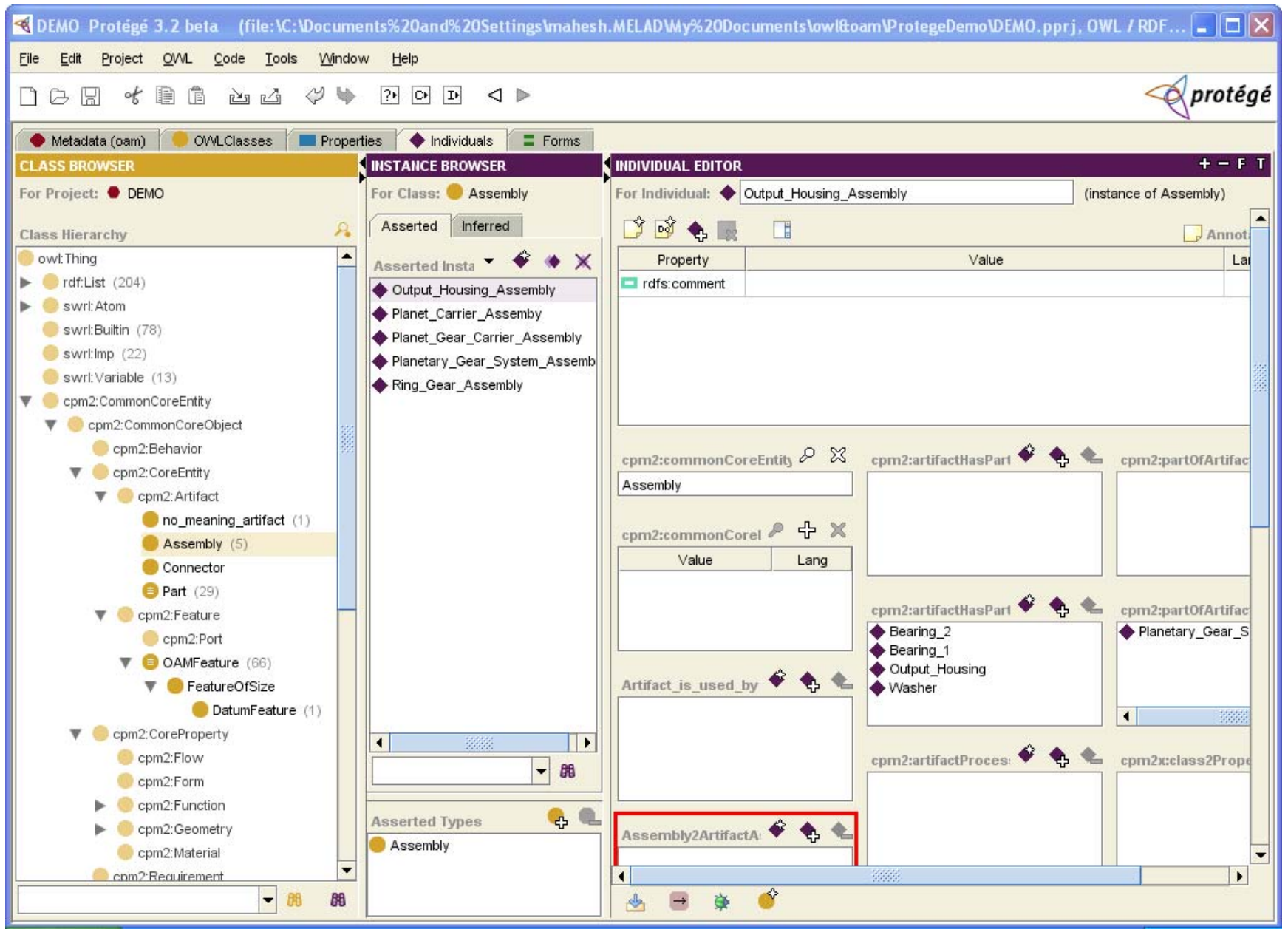

Snapshot A3 Class Browser 